\title{
Impact of DNA Adduct Size, Number, and Relative Position on the Toxicity of Aromatic Amines: A Molecular Dynamics Case Study of ANdG- and APdG-Containing DNA Duplexes
}

\author{
Ryan W. Kung, Trinity K. Deak, Cassidy A. Griffith-Salik, Nathania A. Takyi and Stacey D. \\ Wetmore*
}

\section{Table of Contents}

Figure S1. Structure of ${ }^{\mathrm{AN}} \mathrm{dG}$ in the $\mathrm{W}$ conformation.

Figures S2-S7. MD representative structures across the three 500 ns replicas for ${ }^{A N} d G-$ and ${ }^{A P} d G-$ containing DNA duplexes. S5

Figure S8. Structure of the (undamaged) Narl containing sequence investigated in the present work highlighting the pseudostep between the base pairs used to calculate helical parameters and nucleotide numbering in the modeled helix.

Figures S9-S11. Key dihedral angles of the adduct in monoadducted and diadducted DNA, with standard deviations calculated between replicas.

Figure S12. MD representative structure of $\mathrm{G}_{1}^{B}{ }^{A N} \mathrm{dG}$ monoadducted $\mathrm{DNA}$, highlighting the linking $\mathrm{N}-\mathrm{H}$ hydrogen bonding with $\mathrm{O4}^{\prime}$ of the same nucleotide or $\mathrm{O}^{\prime}$ of the $5^{\prime}$ flanking nucleotide

Figure S13. Histogram of the $\theta$ dihedral angle over all MD replicas for ${ }^{A N} d G$ - and ${ }^{A P} d G$-containing DNA duplexes at $G_{1}, G_{2}$ or $G_{3}$ in monoadducted DNA in the $S$ conformation.....

Figure S14. Different views of overlays of the lesion site from representative structures from all MD simulations of ${ }^{\mathrm{AN}} \mathrm{dG}$ monoadducted DNA in the $\mathrm{S}$ conformation, highlighting the nonplanarity of the bulky group with respect to the nucleobase

Figure S15. LSIE ${ }^{\text {avg }}$ of the adduct in monoadducted and diadducted DNA duplexes, with standard deviations calculated between replicas.

Figure S16. SASA ${ }^{\text {avg }}$ of the adduct in monoadducted and diadducted DNA duplexes, with standard deviations calculated between replicas

Figure S17. MD representative structures of ${ }^{A P} \mathrm{dG}$ monoadducted $D N A$ at $\mathrm{G}_{3}$ and diadducted DNA at $\mathrm{G}_{2} \mathrm{G}_{3}$, highlighting the shift in sugar orientation in the $\mathrm{S}$ conformation at the $\mathrm{G}_{3}$ site.

Figure S18. MD representative structure of ${ }^{A P} d G$ diadducted $D N A$ in the $G_{1}^{S} G_{2}^{S}$ conformer, highlighting the distorted sugar and the intramolecular hydrogen bond between the linker $\mathrm{N}-\mathrm{H}$ and $\mathrm{O}^{\prime}$ in each lesion.

Figure S19. Histograms of the $\theta$ dihedral angle in ${ }^{A N} d G$ and ${ }^{A P} d G$ adducted DNA duplexes in the $S$ conformation at $\mathrm{G}_{1}, \mathrm{G}_{2}$ or $\mathrm{G}_{3}$ over all replicas. 
Figure S20. Different views of overlays of the lesion site from representative structures from all MD simulations of ${ }^{\mathrm{AN}} \mathrm{dG}$ diadducted DNA in the $\mathrm{S}$ conformation, highlighting the nonplanarity of the bulky group with respect to the nucleobase.

Figure S21. Structures of $A$ ) the $G_{1}^{B}$ (left), $G_{3}^{B}$ (middle) and $G_{1}^{B} G_{3}^{B}$ (right) ${ }^{A N} d G$-containing helices and $B$ ) the $\mathrm{G}_{2}^{S}$ (left), $\mathrm{G}_{3}^{S}$ (middle) and $\mathrm{G}_{2}^{S} \mathrm{G}_{3}^{S}$ (right) ${ }^{A P} \mathrm{dG}$-containing helices, highlighting the range in helical distortion observed for monoadducted and diadducted DNA duplexes.

Table S1. Average (standard deviation in parentheses) backbone RMSD ( $(\AA)$ across MD simulations of damaged DNA.

Table S2. Occupancies (\%) of the Watson-Crick hydrogen bonds at the terminal ends of DNA helices. S23

Tables S3-S8. Occupancies (\%) of the Watson-Crick hydrogen bonds at the lesion site and flanking base pairs for monoadducted and diadducted DNA duplexes.

Table S9-S11. Averages and standard deviations in key adduct dihedral angles (deg.) for each replica and across all replicas for monoadducted DNA.

Table S12. Occupancies (\%) of the non-Watson-Crick hydrogen bonds at the lesion site of monoadducted DNA.

Table S13. Average (standard deviation in parentheses) lesion site interaction energy ( $\mathrm{kJ} / \mathrm{mol})$ for undamaged and monoadducted DNA duplexes.

Table S14. Average (standard deviation in parentheses) pseudostep parameters for undamaged and monoadducted DNA.

Table S15. Relative deviations in the pseudostep parameters from natural DNA, with the number of Xs representing the degree of distortion.

Table S16. Average (standard deviation in parentheses) solvent accessible surface area of the hydrophobic aryl bulky group (SASA, $\AA^{2}$ ) for monoadducted DNA.

Tables S17-S19. Averages and standard deviations in key adduct dihedral angles (deg.) for each replica and across all replicas for diadducted DNA.

Table S20. Average (standard deviation in parentheses) solvent accessible surface area of the hydrophobic aryl bulky group (SASA, $\AA^{2}$ ) for diadducted DNA.

Table S21. Occupancies (\%) for the non-Watson-Crick hydrogen bonds at the diadducted DNA lesion sites in the B conformation.

Table S22. Average (standard deviation in parentheses) pseudostep parameters within diadducted DNA.

Table S23. Occupancies (\%) for the non-Watson-Crick hydrogen bonds at the diadducted DNA damage sites in the $\mathrm{S}$ conformation.

Tables S24, S25. Average (standard deviation in parentheses) lesion site interaction energies $(\mathrm{kJ} / \mathrm{mol})$ for monoadducted and diadducted DNA duplexes in the B or S conformations. 
Table S26. Average $\Delta\left(\right.$ SASA $\left.^{\text {avg }}\right)$ and $\Delta\left(\right.$ LSIE $\left.^{\text {avg }}\right)$ upon the addition of a second lesion............................ S46

Tables S27, S28. MOL2 file containing partial charges and atom types of the ${ }^{A N} \mathrm{dG}$ and ${ }^{A P} \mathrm{dG}$ adducts used for $\mathrm{MD}$ simulations (atom numbers are provided in the associated figure). 


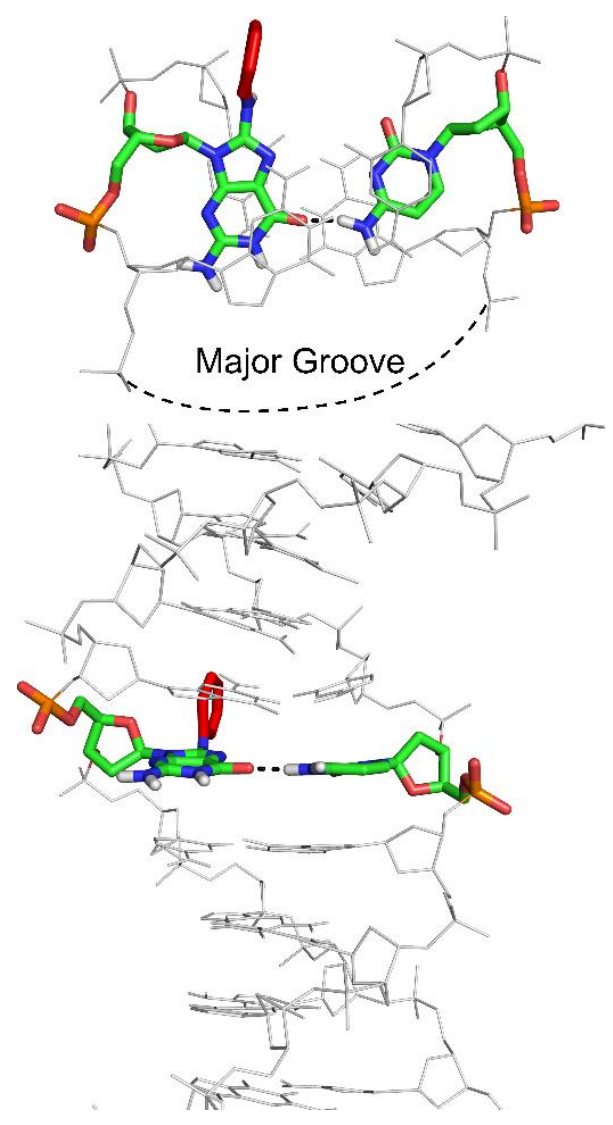

Figure S1: Structure of ${ }^{A N} \mathrm{dG}$ adducted DNA in the $\mathrm{W}$ conformation, with the major groove highlighted and the bulky aryl group (red) in the minor groove. 
A)

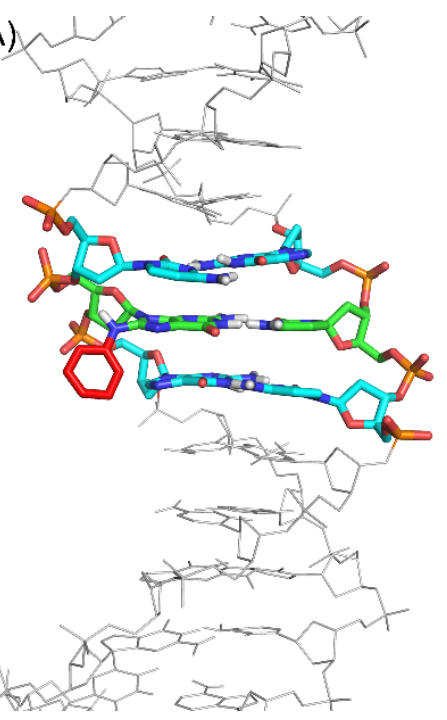

D)

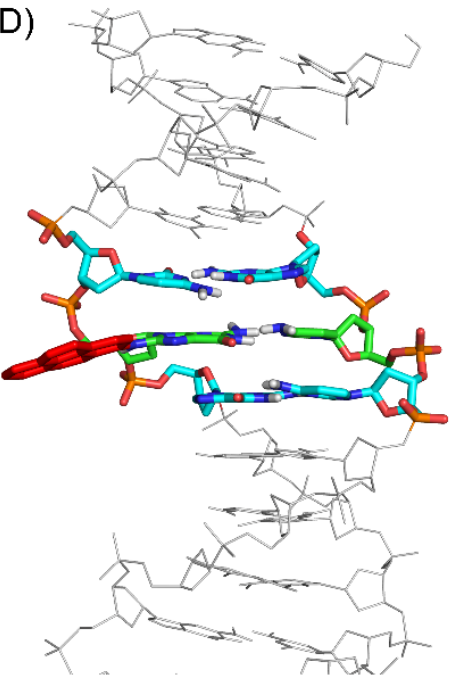

B)

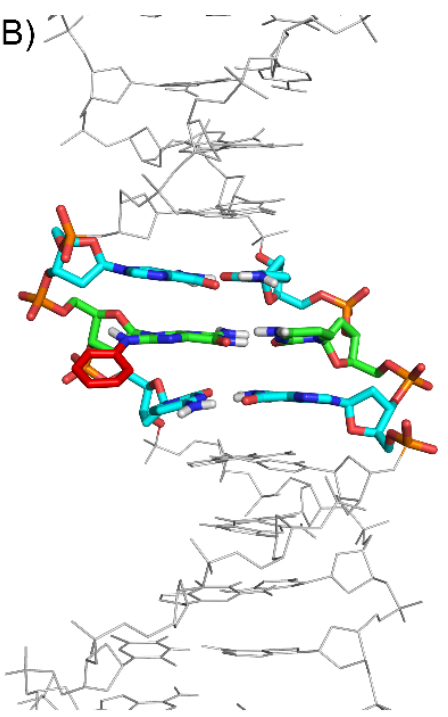

E)

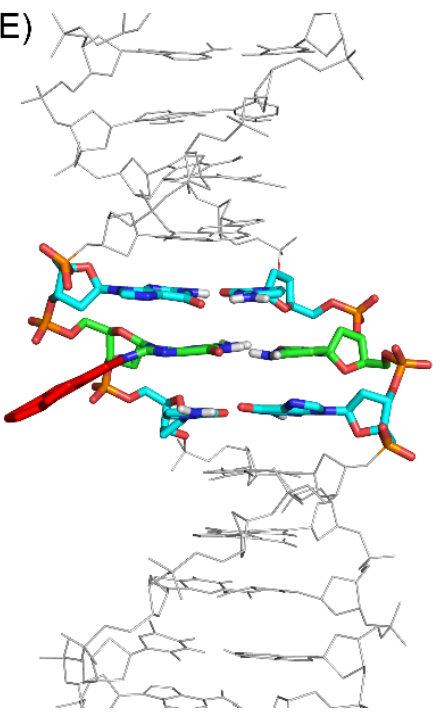

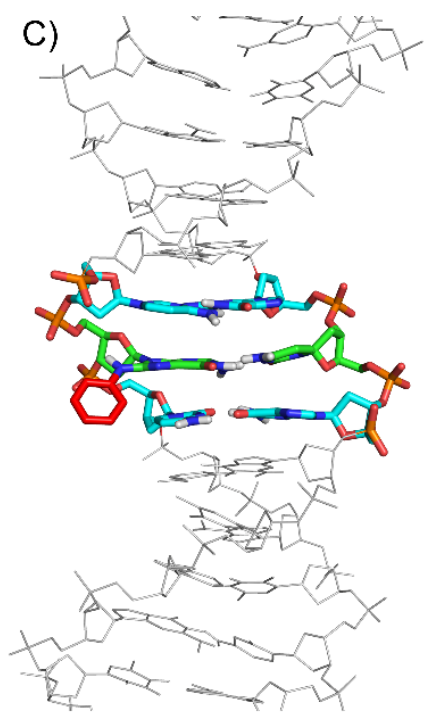

F)

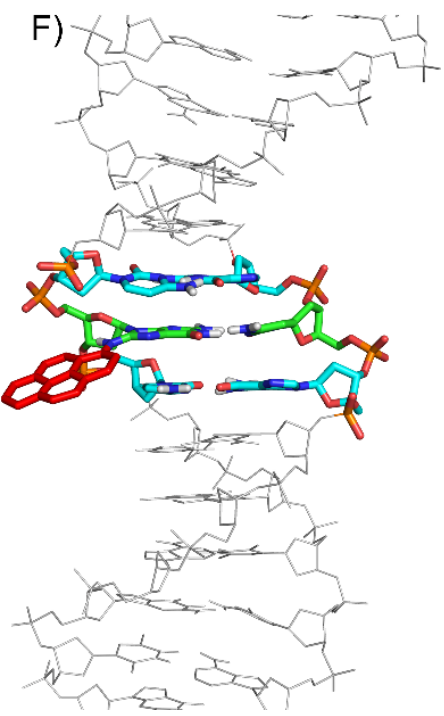

Figure S2: MD representative structures across the three 500 ns replicas for ${ }^{A N} d G$ (top) or ${ }^{A P} d G$ (bottom) monoadducted DNA at $G_{1}$ (left, $A, D$ ), $G_{2}$ (middle $B, E$ ) or $G_{3}$ (right, $C, F$ ) in the B conformation. The adducted base pair is shown in green, the bulky moiety in red, and the flanking base pairs in cyan. 
A)
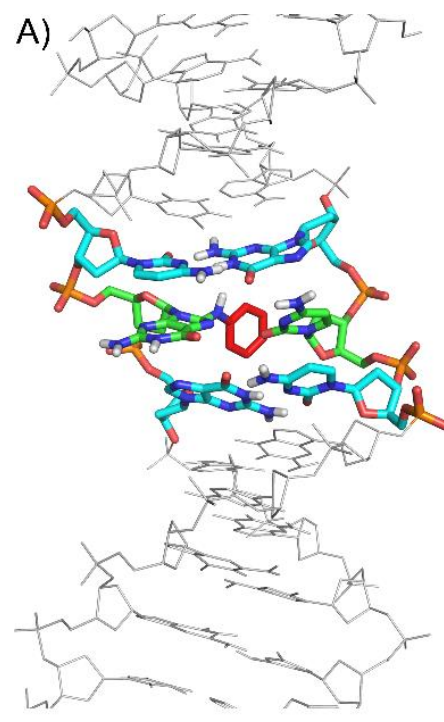

D)
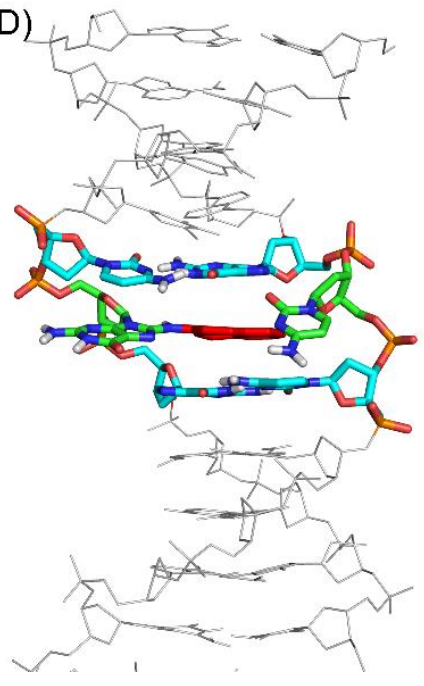

B)

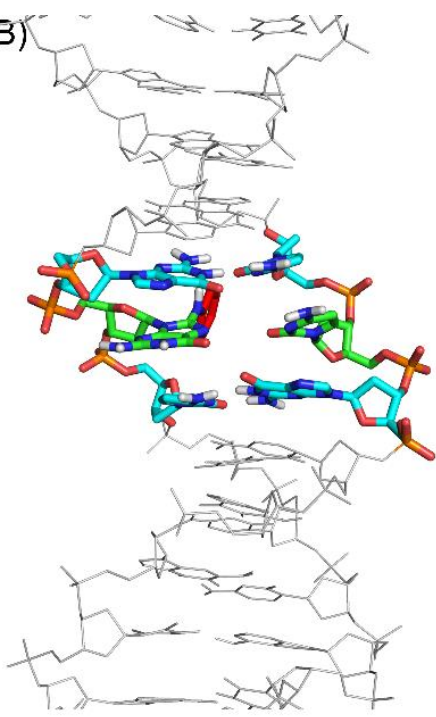

E)

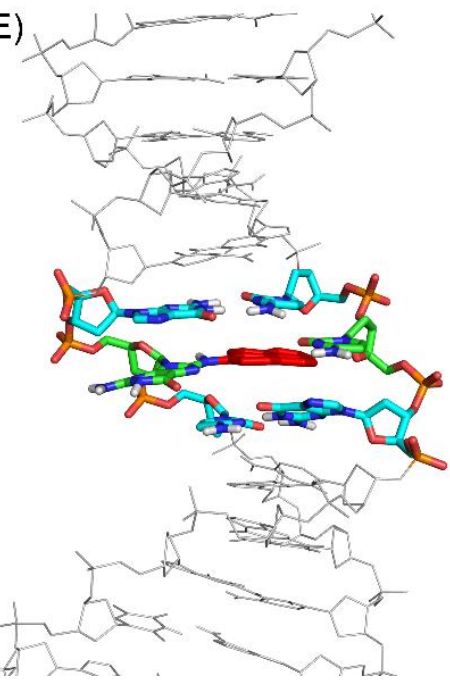

C)
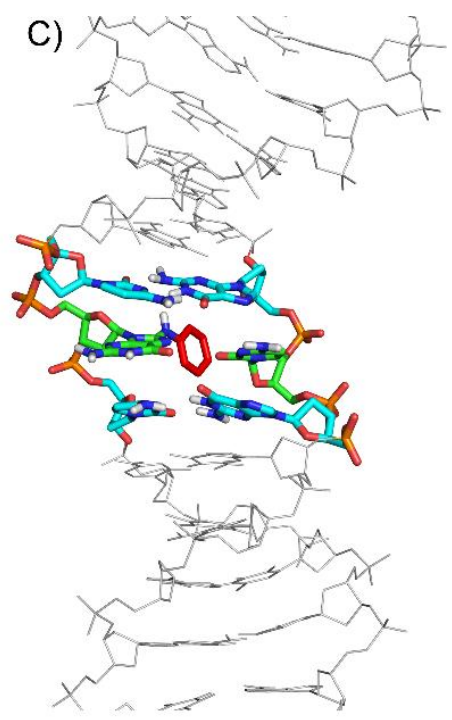

F)

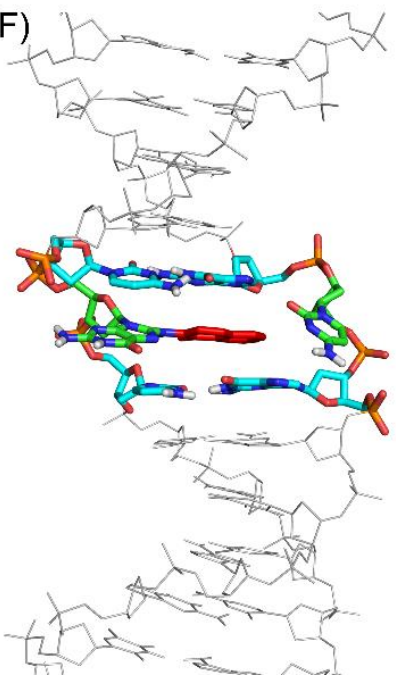

Figure S3: MD representative structures across the three 500 ns replicas for ${ }^{A N} d G$ (top) or ${ }^{A P} d G$ (bottom) monoadducted DNA at $\mathrm{G}_{1}$ (left, $\mathrm{A}, \mathrm{D}$ ), $\mathrm{G}_{2}$ (middle $\mathrm{B}, \mathrm{E}$ ) or $\mathrm{G}_{3}$ (right, $\mathrm{C}, \mathrm{F}$ ) in the $\mathrm{S}$ conformation. The adducted base pair is shown in green, the bulky moiety in red, and the flanking base pairs in cyan. 
A)

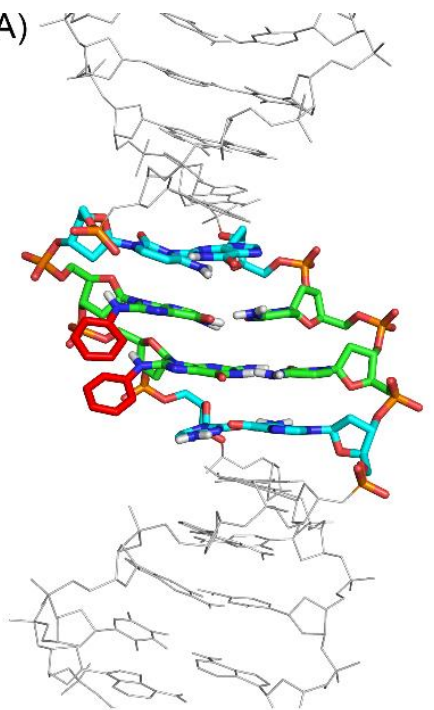

D)

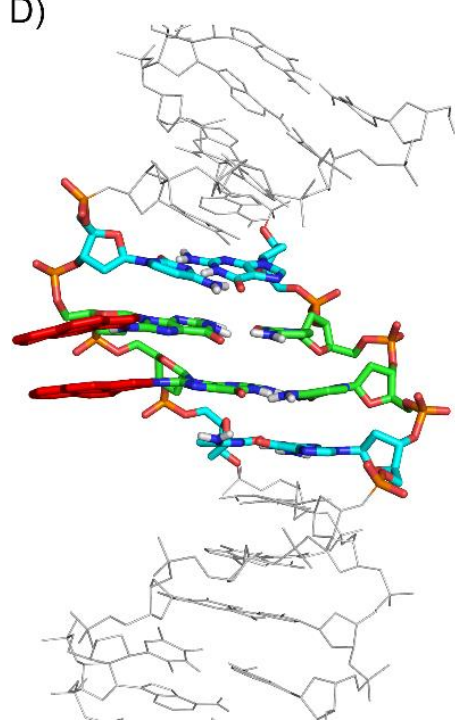

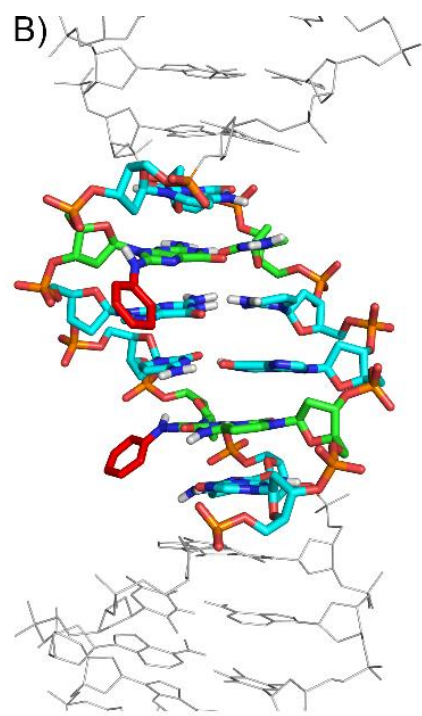

E)

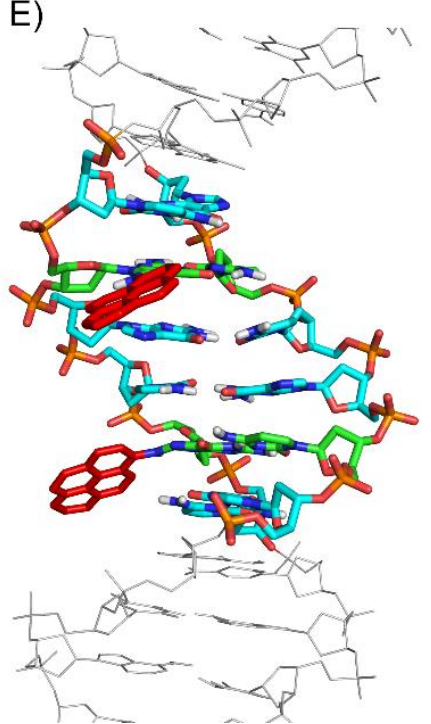

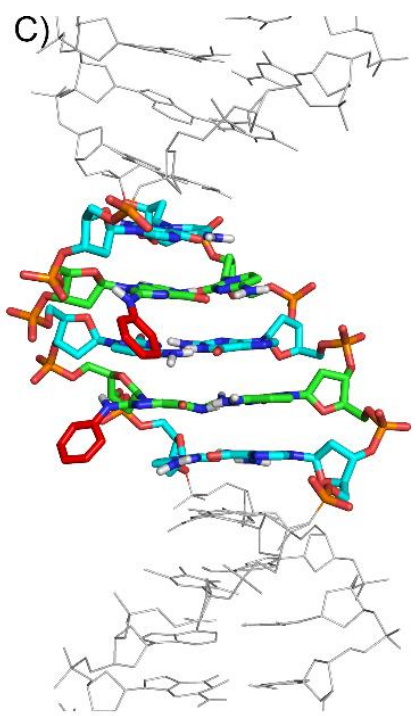

F)

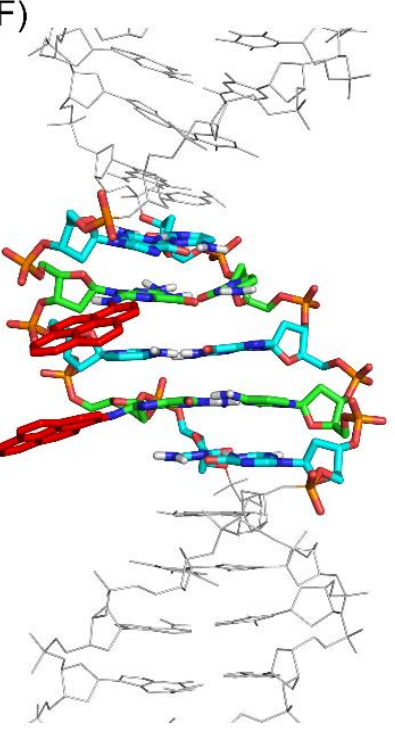

Figure S4: MD representative structures across the three 500 ns replicas for ${ }^{A N} d G$ (top) or ${ }^{A P} d G$ (bottom) diadducted $D N A$ in the $G_{1}^{B} G_{2}^{B}$ (left, $A, D$ ), $G_{1}^{B} G_{3}^{B}$ (middle, $B, E$ ) or $G_{2}^{B} G_{3}^{B}$ (right, $C, F$ ) conformations. The adducted base pair is shown in green, the bulky moiety in red, and the flanking base pairs in cyan. 
A)
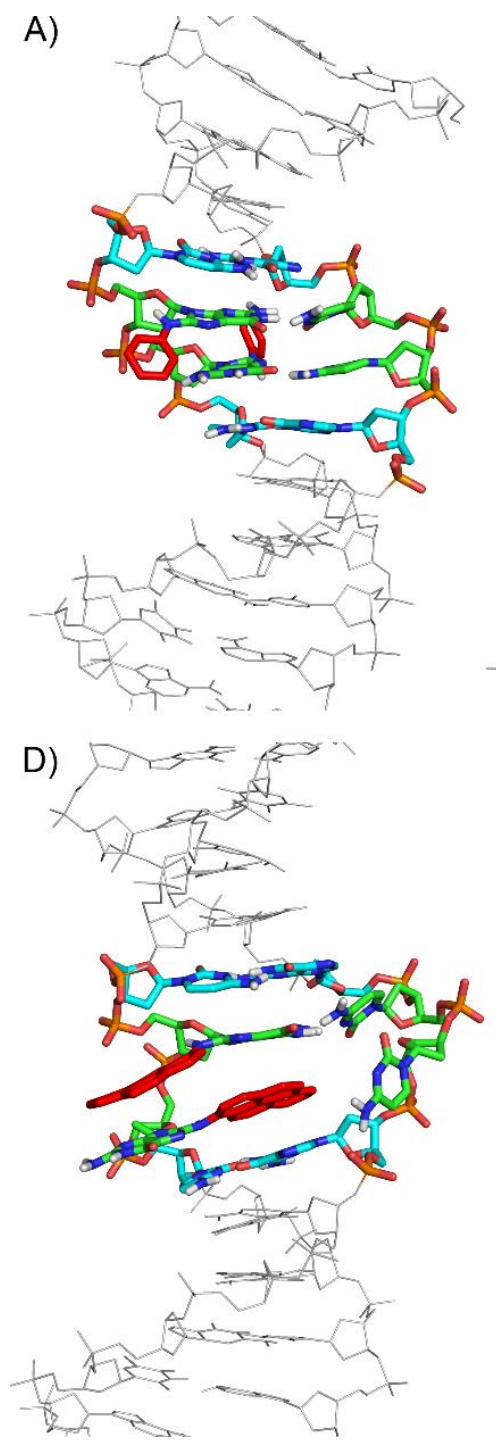

B)
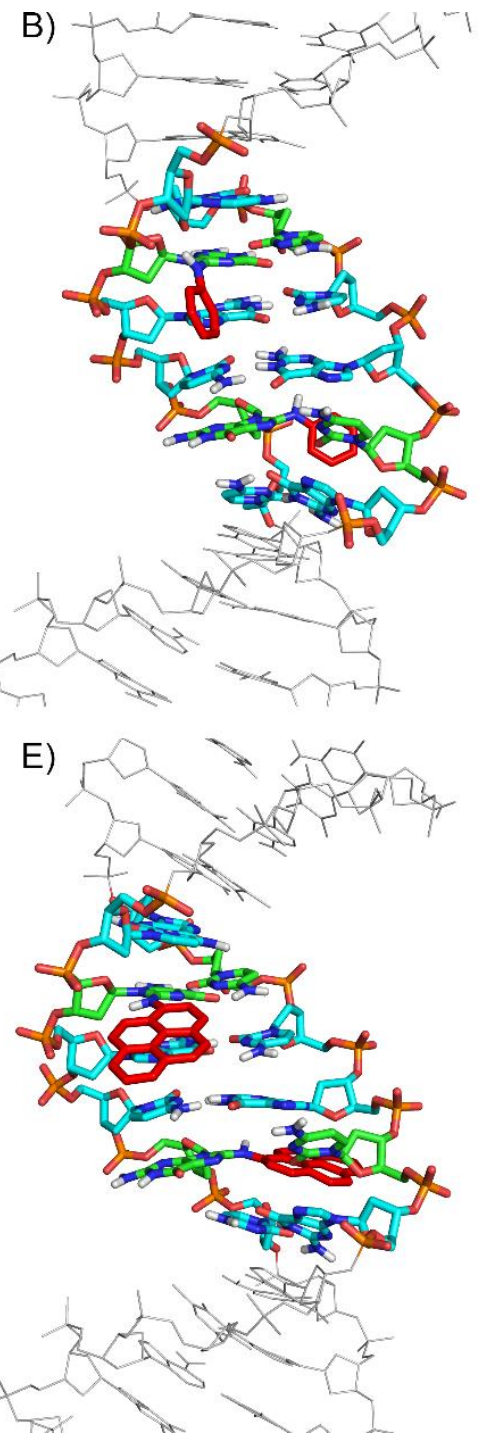

C)
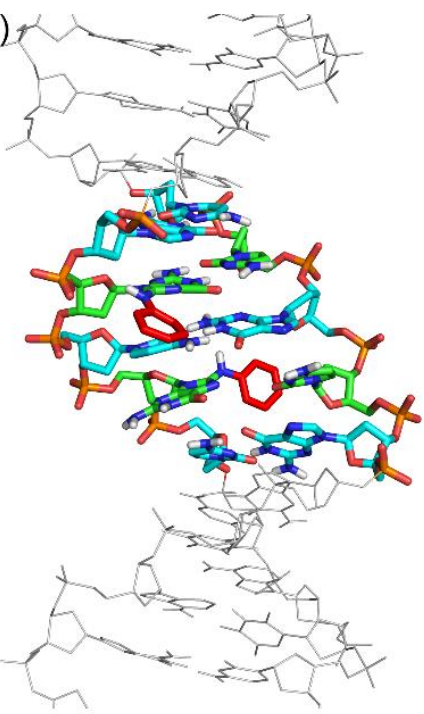

F)
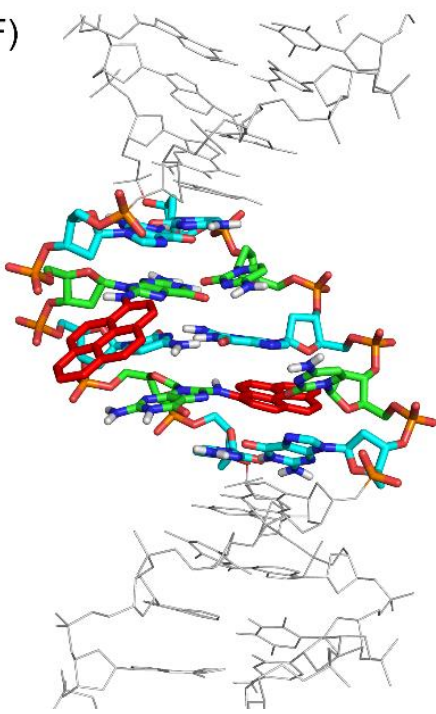

Figure S5: MD representative structures across the three $500 \mathrm{~ns}$ replicas for ${ }^{\mathrm{AN}} \mathrm{dG}$ (top) or ${ }^{\mathrm{AP}} \mathrm{dG}$ (bottom) diadducted $D N A$ in the $G_{1}^{B} G_{2}^{S}$ (left, $A, D$ ), $G_{1}^{B} G_{3}^{S}$ (middle, $B, E$ ) or $G_{2}^{B} G_{3}^{S}$ (right, $C, F$ ) conformations. The adducted base pair is shown in green, the bulky moiety in red, and the flanking base pairs in cyan. 
A)

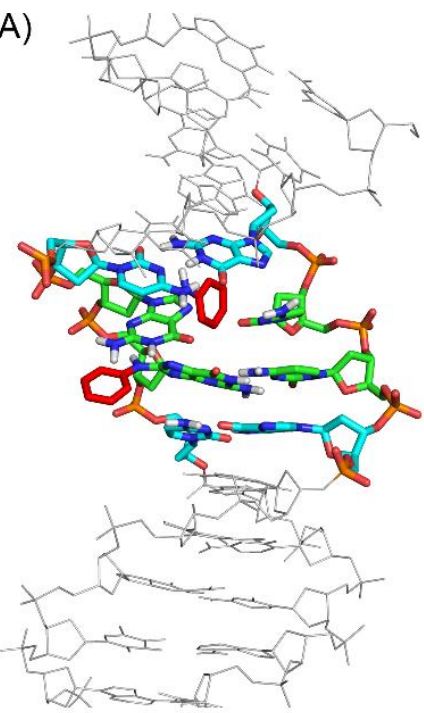

D)
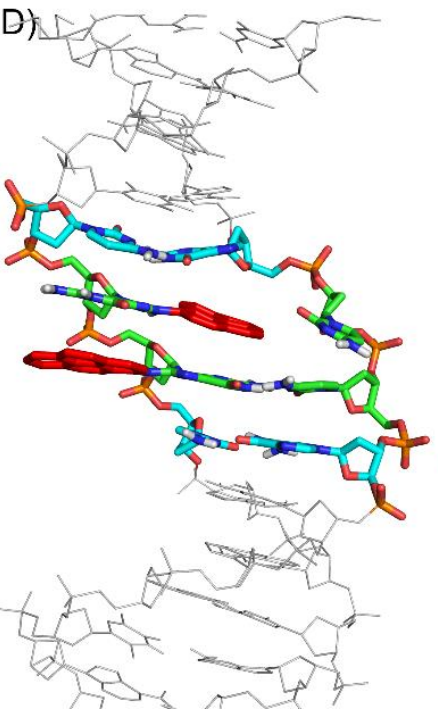

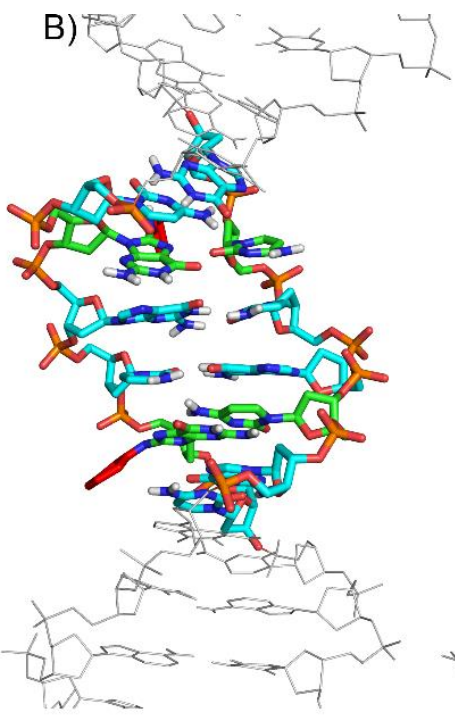

C)
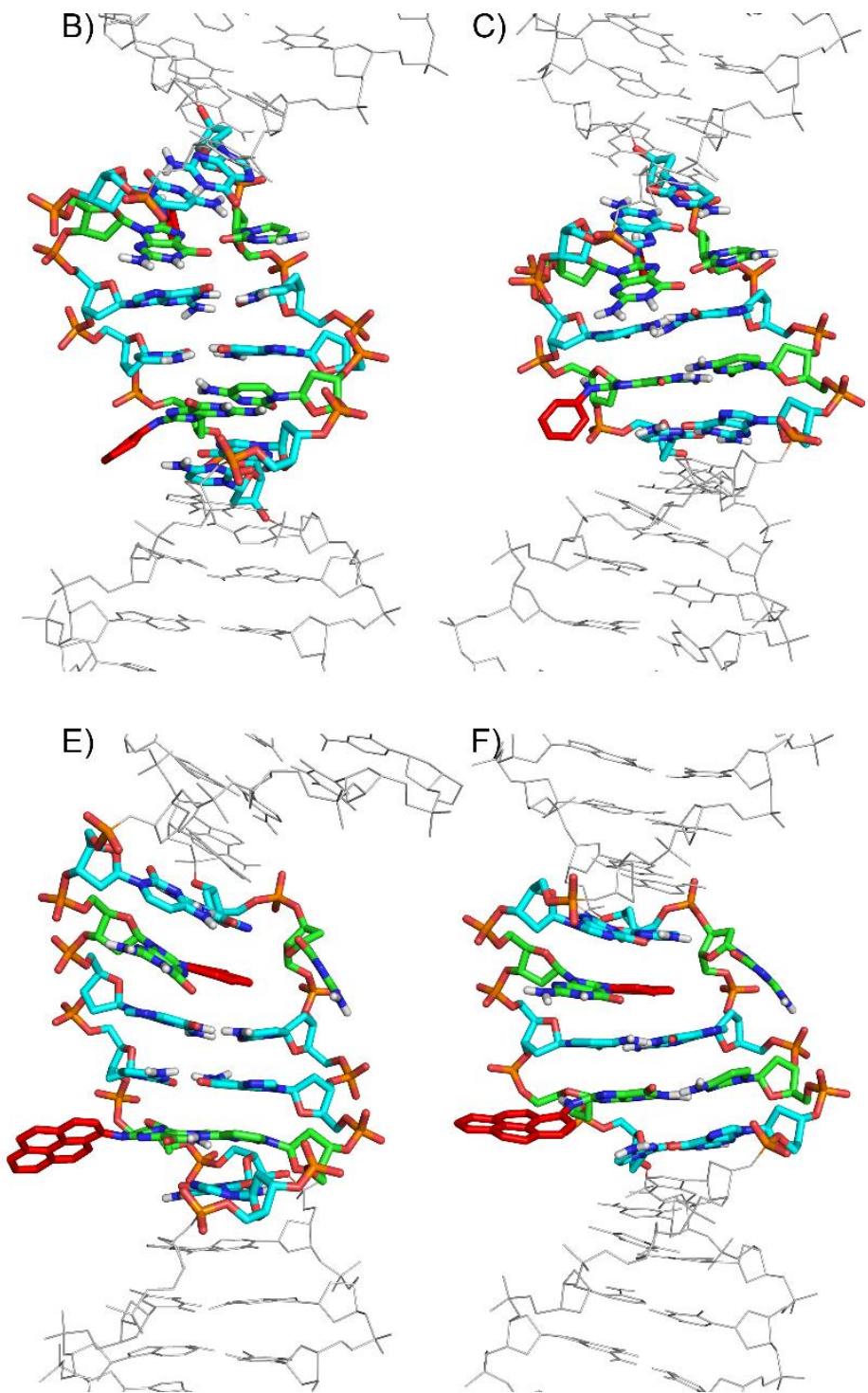

Figure S6: MD representative structures across the three 500 ns replicas for ${ }^{A N} d G$ (top) or ${ }^{A P} d G$ (bottom) diadducted DNA in the $G_{1}^{S} G_{2}^{B}$ (left, $A, D$ ), $G_{1}^{S} G_{3}^{B}$ (middle, $B, E$ ) or $G_{2}^{S} G_{3}^{B}$ (right, $C, F$ ) conformations. The adducted base pair is shown in green, the bulky moiety in red, and the flanking base pairs in cyan. 
A)

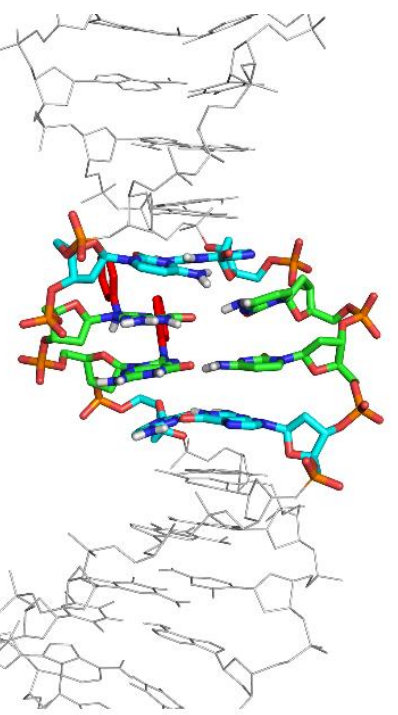

D)

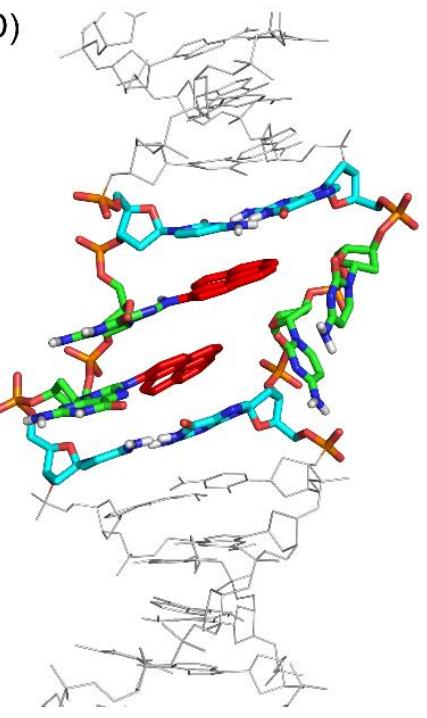

B)

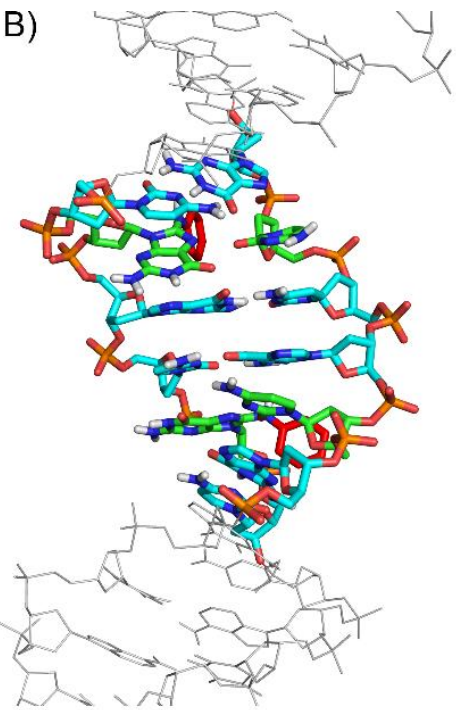

E)

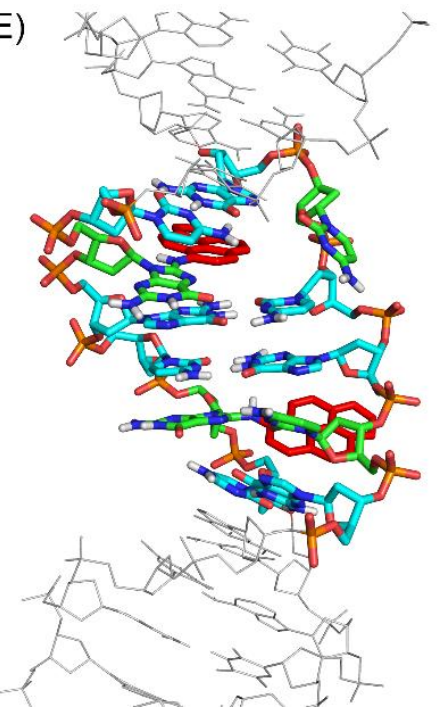

C)
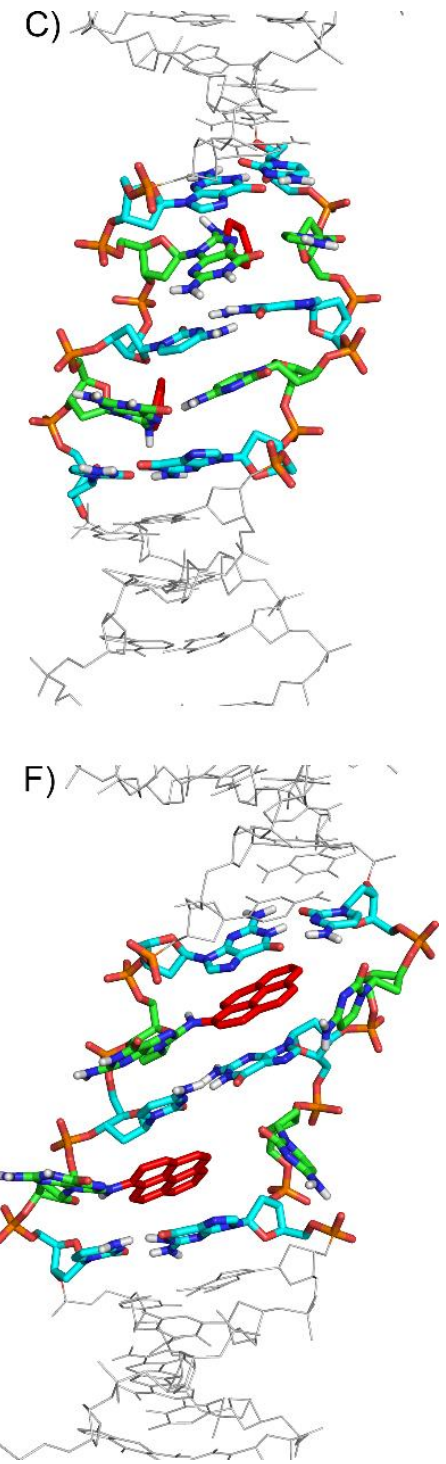

Figure S7: MD representative structures across the three $500 \mathrm{~ns}$ replicas for ${ }^{\mathrm{AN}} \mathrm{dG}$ (top) or ${ }^{\mathrm{AP}} \mathrm{dG}$ (bottom) diadducted DNA in the $G_{1}^{S} G_{2}^{S}$ (left, $A, D$ ), $G_{1}^{S} G_{3}^{S}$ (middle, $B, E$ ) or $G_{2}^{S} G_{3}^{S}$ (right, $C, F$ ) conformations. The adducted base pair is shown in green, the bulky moiety in red, and the flanking base pairs in cyan. 

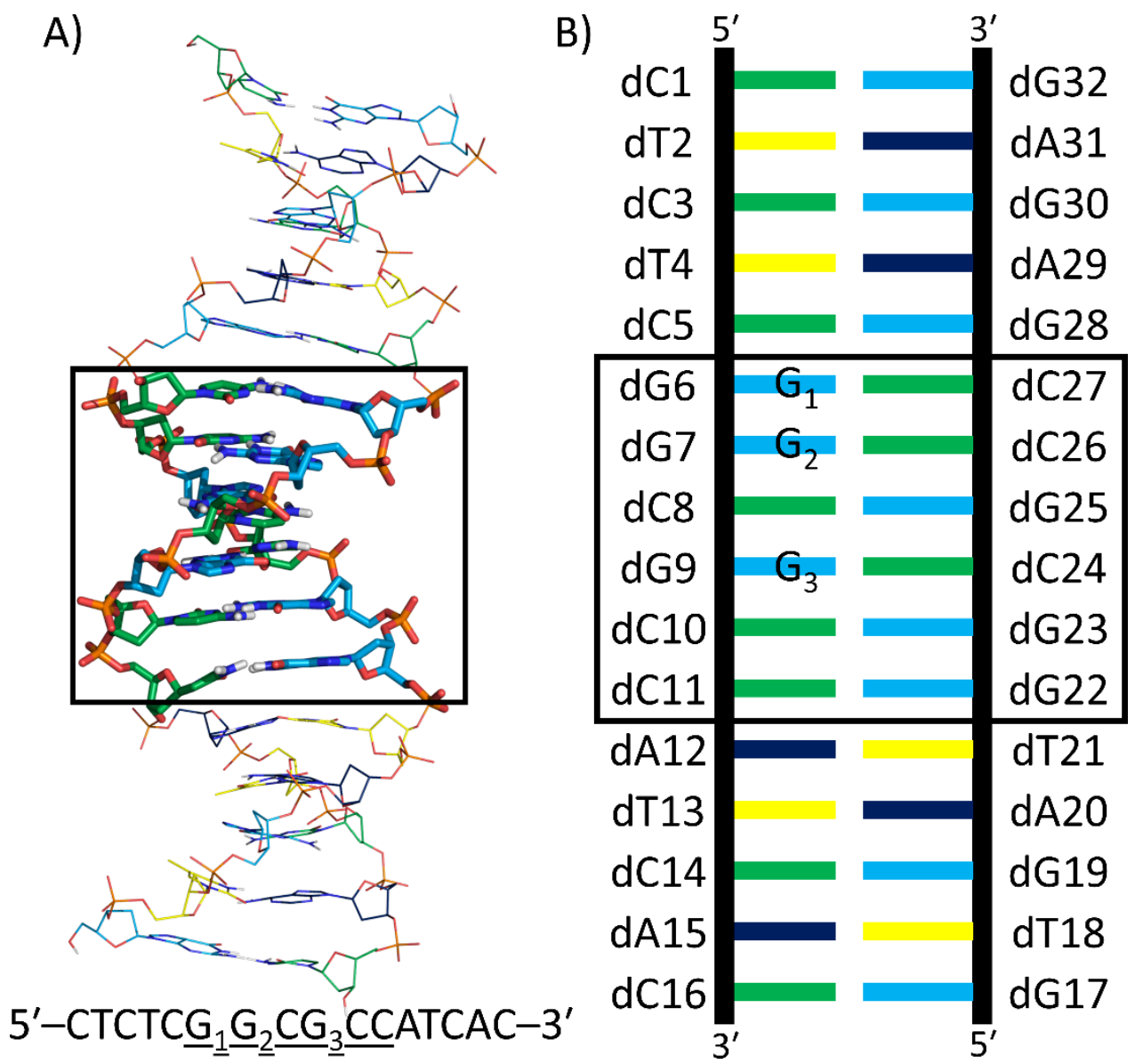

Figure S8: A) Structure and B) nucleotide numbering of the (undamaged) Narl containing sequence investigated in the present work. Helical parameters were calculated using a pseudostep between the base pairs on either side of the box. 
360

300

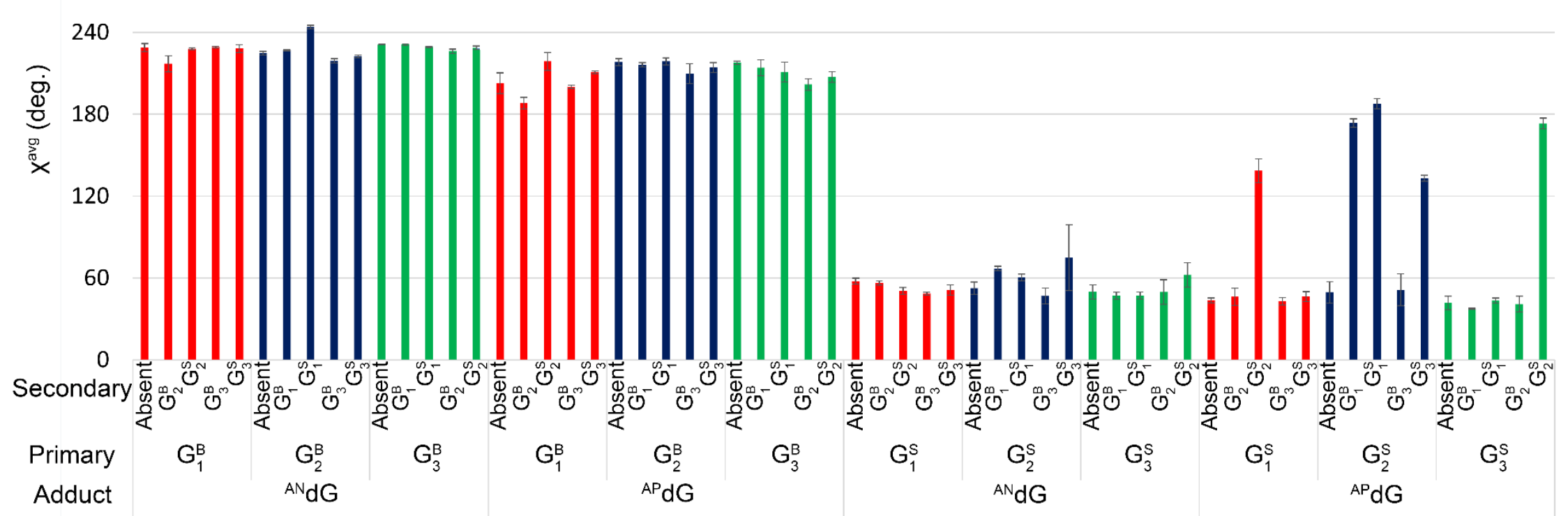

Figure S9: $\chi^{\text {avg }}$ (deg.) of the adduct in monoadducted (Secondary = Absent) and diadducted DNA, with standard deviations calculated between replicas. The bars are color coded based on the lesion under investigation, with the $G_{1}$ site in red, $G_{2}$ site in green and $G_{3}$ site in blue (see main text Figure 1 for $\chi$ definition). 
360

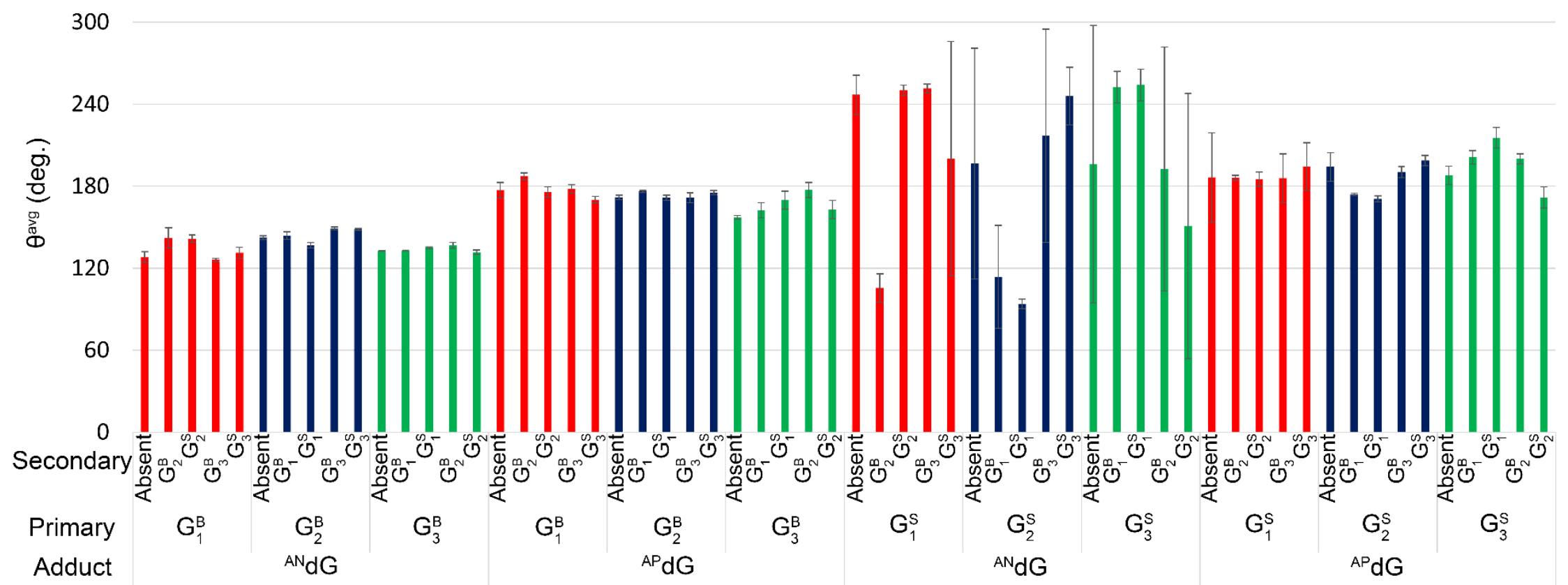

Figure S10: $\theta^{\text {avg }}$ (deg.) of the adduct in monoadducted (Secondary = Absent) and diadducted DNA, with standard deviations calculated between replicas. The bars are color coded based on the lesion under investigation, with the $G_{1}$ site in red, $G_{2}$ site in green and $G_{3}$ site in blue (see main text Figure 1 for $\theta$ definition). 
300

240

180

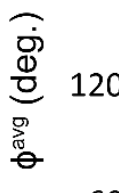

60

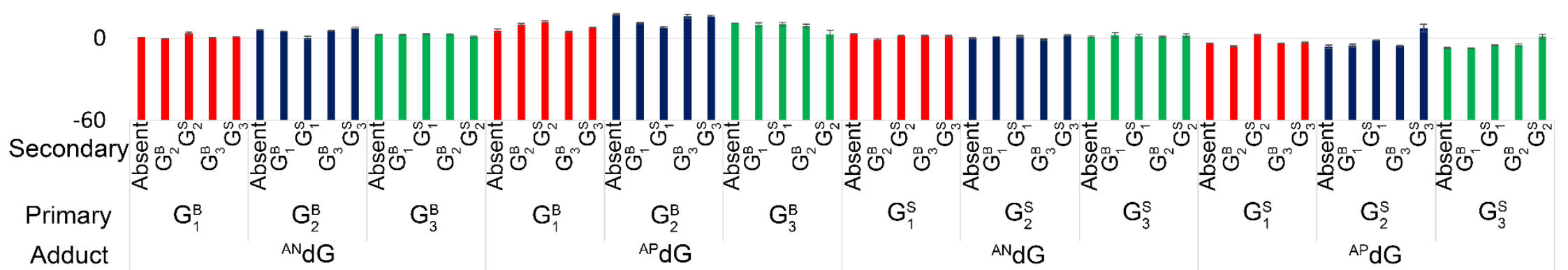

Figure S11: $\phi^{\text {avg }}$ (deg.) of the adduct in monoadducted (Secondary = Absent) and diadducted DNA, with standard deviations calculated between replicas. The bars are color coded based on the lesion under investigation, with the $G_{1}$ site in red, $G_{2}$ site in green and $G_{3}$ site in blue (see main text Figure 1 for $\phi$ definition). 

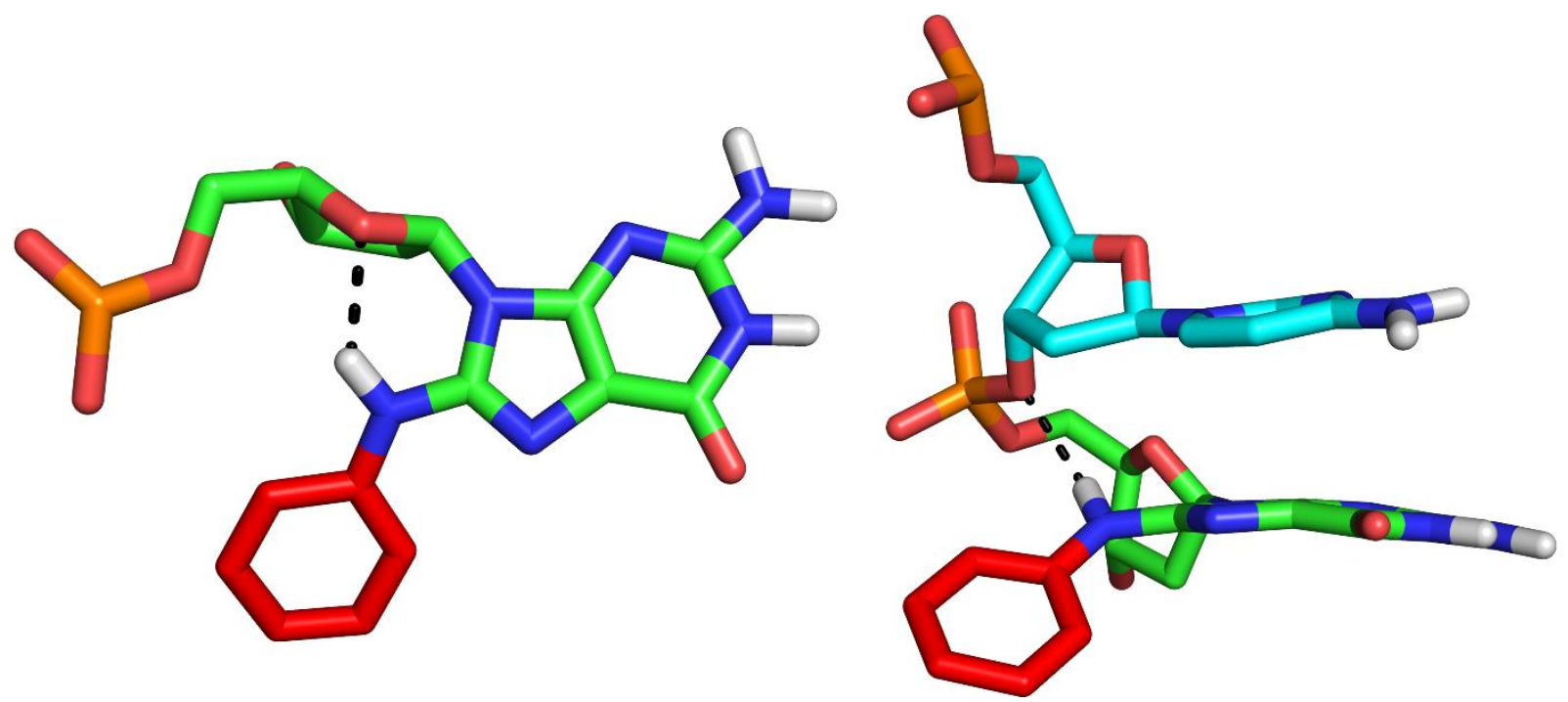

Figure S12: $M D$ representative structure of $\mathrm{G}_{1}^{\mathrm{B}} \mathrm{AN}_{\mathrm{dG}}$ monoadducted $\mathrm{DNA}$, highlighting the linking $\mathrm{N}-\mathrm{H}$ hydrogen bonding with $\mathrm{O}^{\prime}$ ' of the same nucleotide seen for both ${ }^{\mathrm{AN}} \mathrm{dG}$ and ${ }^{\mathrm{AP}} \mathrm{dG}$ at all positions (left) or ${ }^{O 3^{\prime}}$ of the $5^{\prime}$ flanking nucleotide seen for ${ }^{A N} d G$ and $G_{1}$ (right). The adducted base is shown in green, the bulky moiety in red, and the flanking base in cyan. 


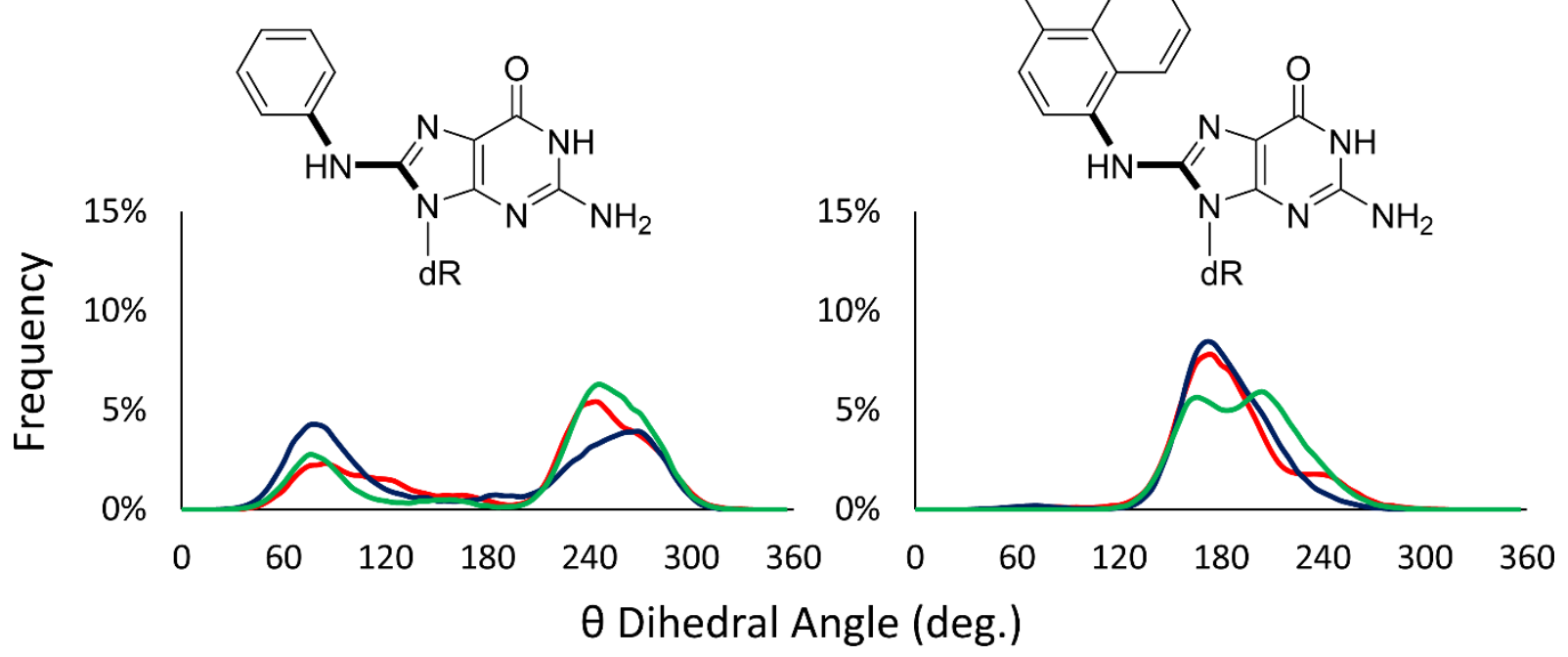

Figure S13: Histogram of the $\theta$ dihedral angle (bolded) over all MD replicas for ${ }^{A N} \mathrm{dG}$ - (left) and ${ }^{\text {AP }}$ dGcontaining DNA duplexes (right) at $G_{1}$ (red), $G_{2}$ (blue) or $G_{3}$ (green) in monoadducted DNA in the $S$ conformation.
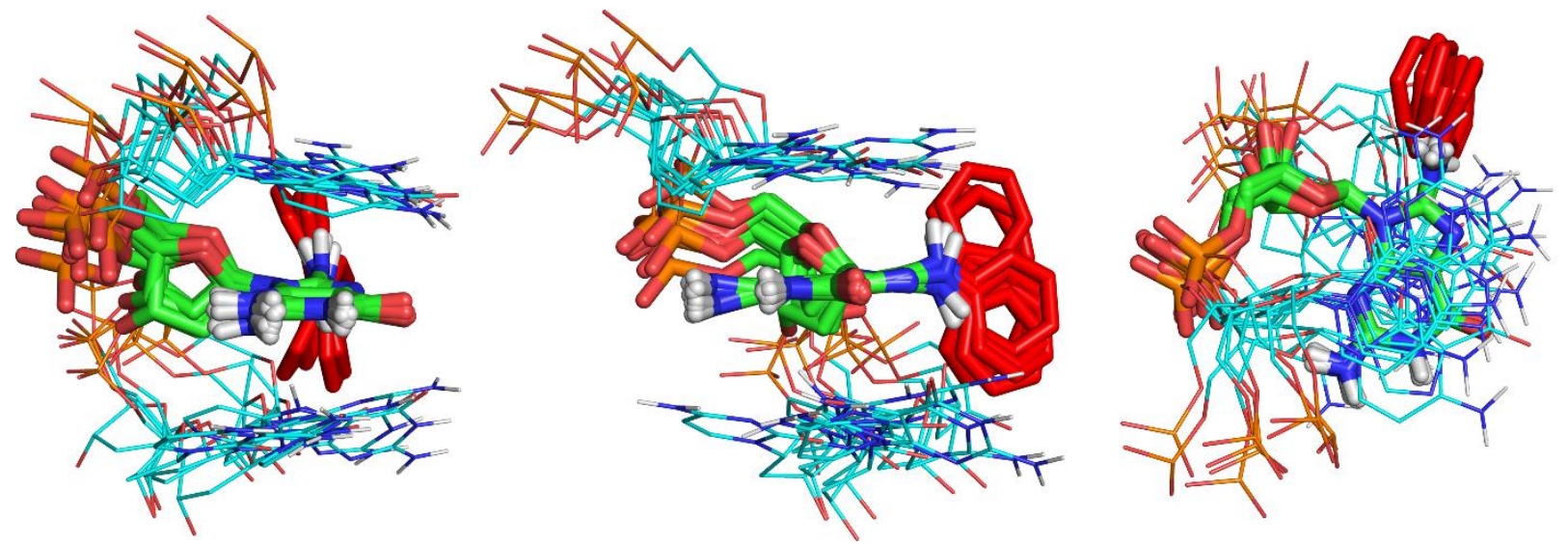

Figure S14: Different views of an overlay of the lesion site from representative structures obtained from each MD simulation of ${ }^{A N} \mathrm{dG}$ monoadducted DNA in the $\mathrm{S}$ conformation, highlighting the nonplanarity of the bulky group with respect to the nucleobase. The adducted base is shown in green, the bulky moiety in red, and the flanking bases in cyan. 
100

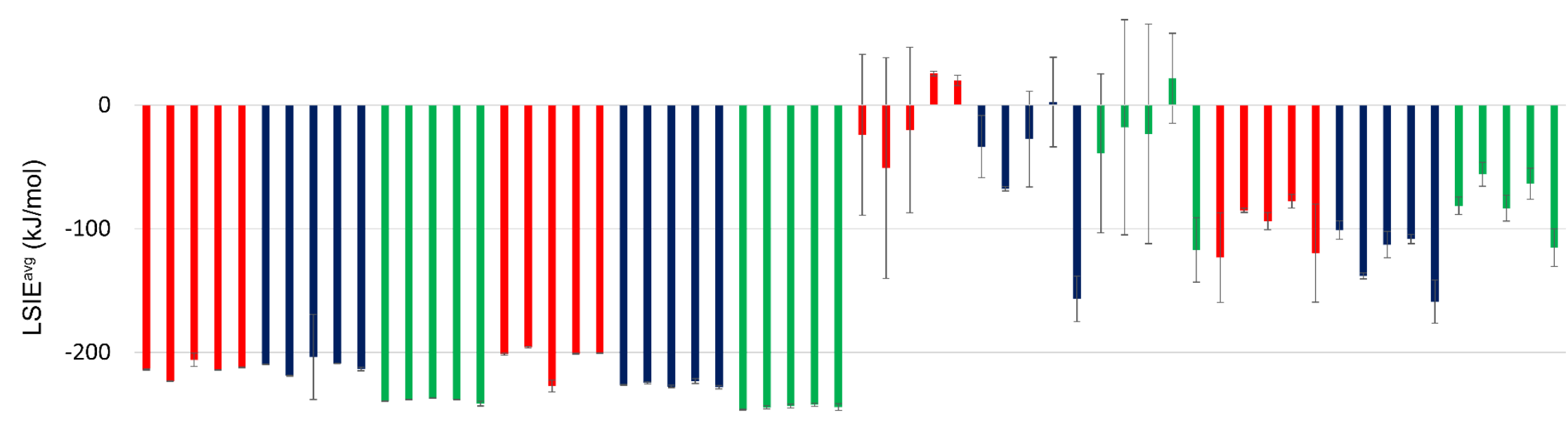

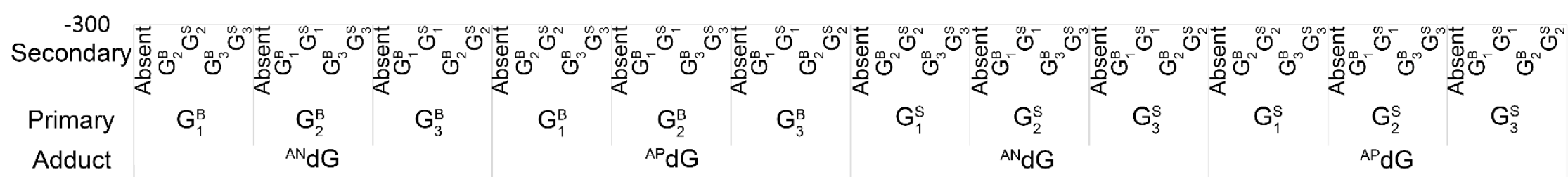

Figure S15: LSIE ${ }^{\text {avg }}(\mathrm{kJ} / \mathrm{mol})$ of the adduct in monoadducted (Secondary = Absent) and diadducted DNA duplexes, with standard deviations calculated between replicas. The bars are color coded based on the lesion under investigation, with the $\mathrm{G}_{1}$ site in red, $\mathrm{G}_{2}$ site in green and $\mathrm{G}_{3}$ site in blue. 


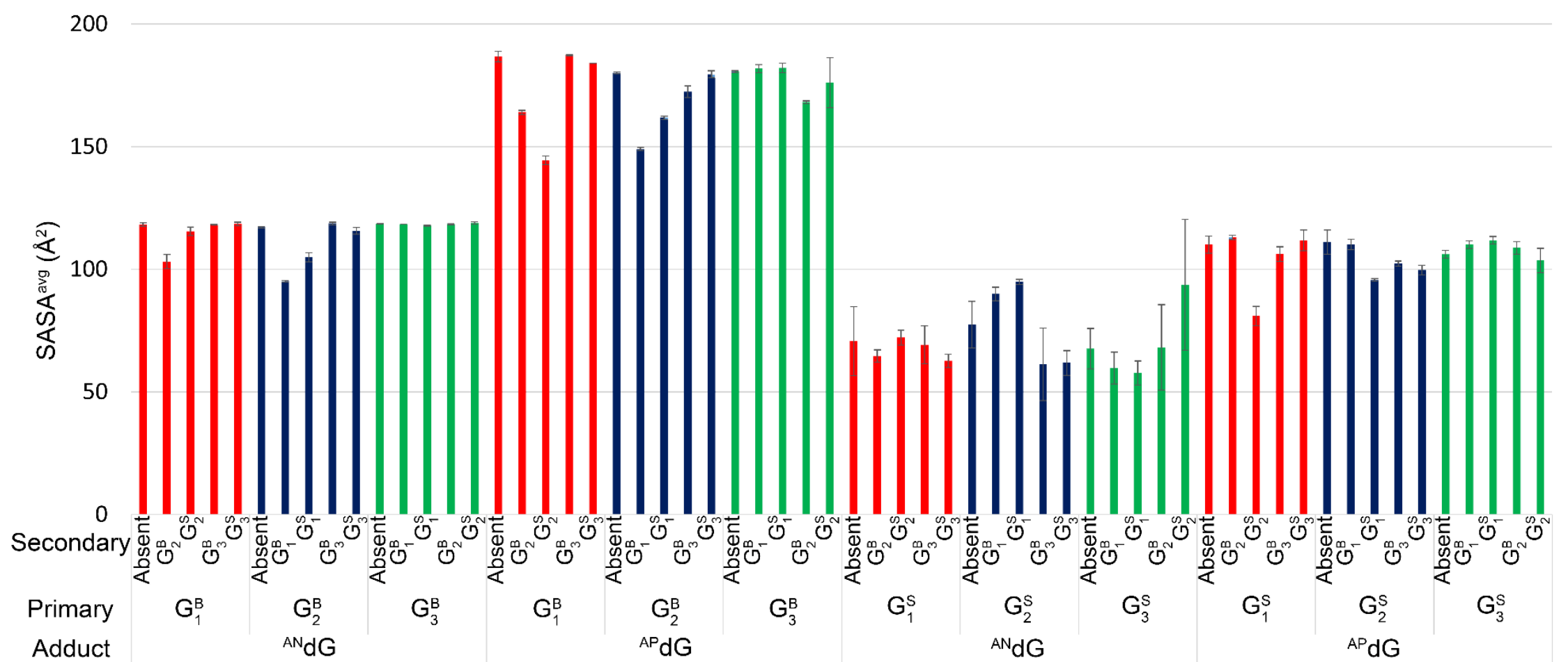

Figure S16: SASA ${ }^{\text {avg }}\left(\AA^{2}\right)$ of the adduct in monoadducted (Secondary = Absent) and diadducted DNA duplexes, with standard deviations calculated between replicas. The bars are color coded based on the lesion under investigation, with the $G_{1}$ site in red, $G_{2}$ site in green and $G_{3}$ site in blue. 

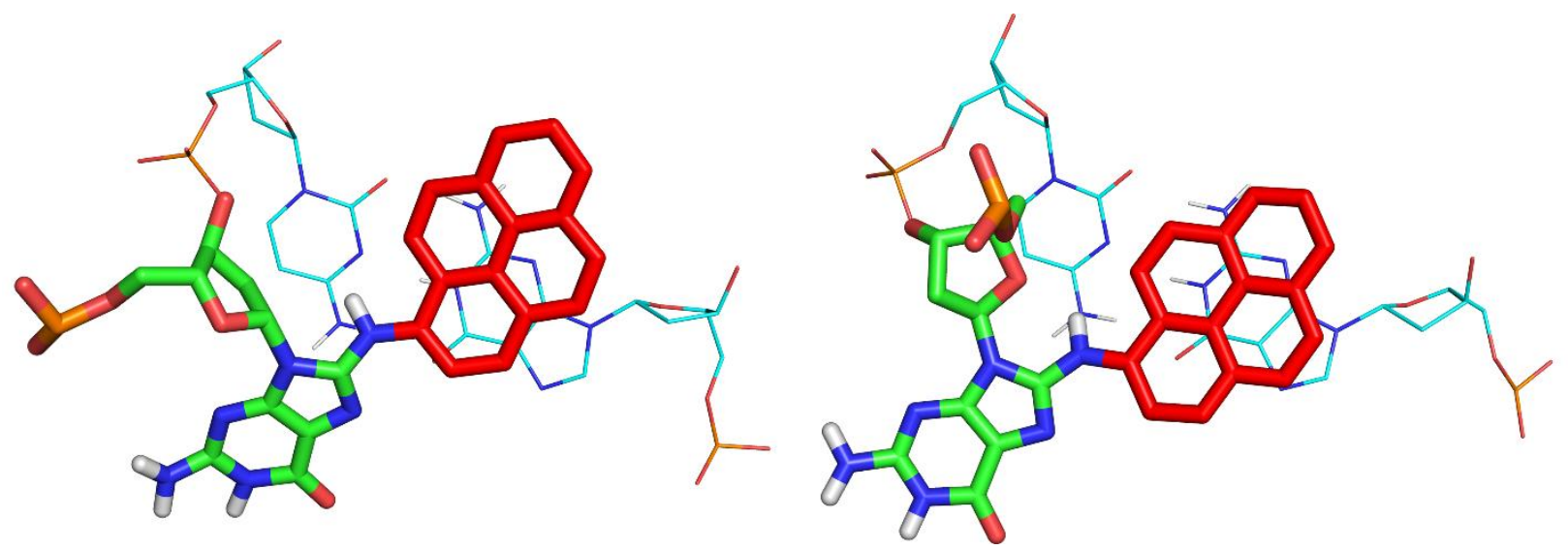

Figure S17: MD representative structures of ${ }^{A P} \mathrm{dG}$ monoadducted DNA at $\mathrm{G}_{3}$ (left) and diadducted DNA at $\mathrm{G}_{2} \mathrm{G}_{3}$ (right), highlighting the shift in sugar orientation in the $S$ conformation at $\mathrm{G}_{3}$ upon addition of the second lesion. The adducted base is shown in green, the bulky moiety in red, and the flanking bases in cyan.

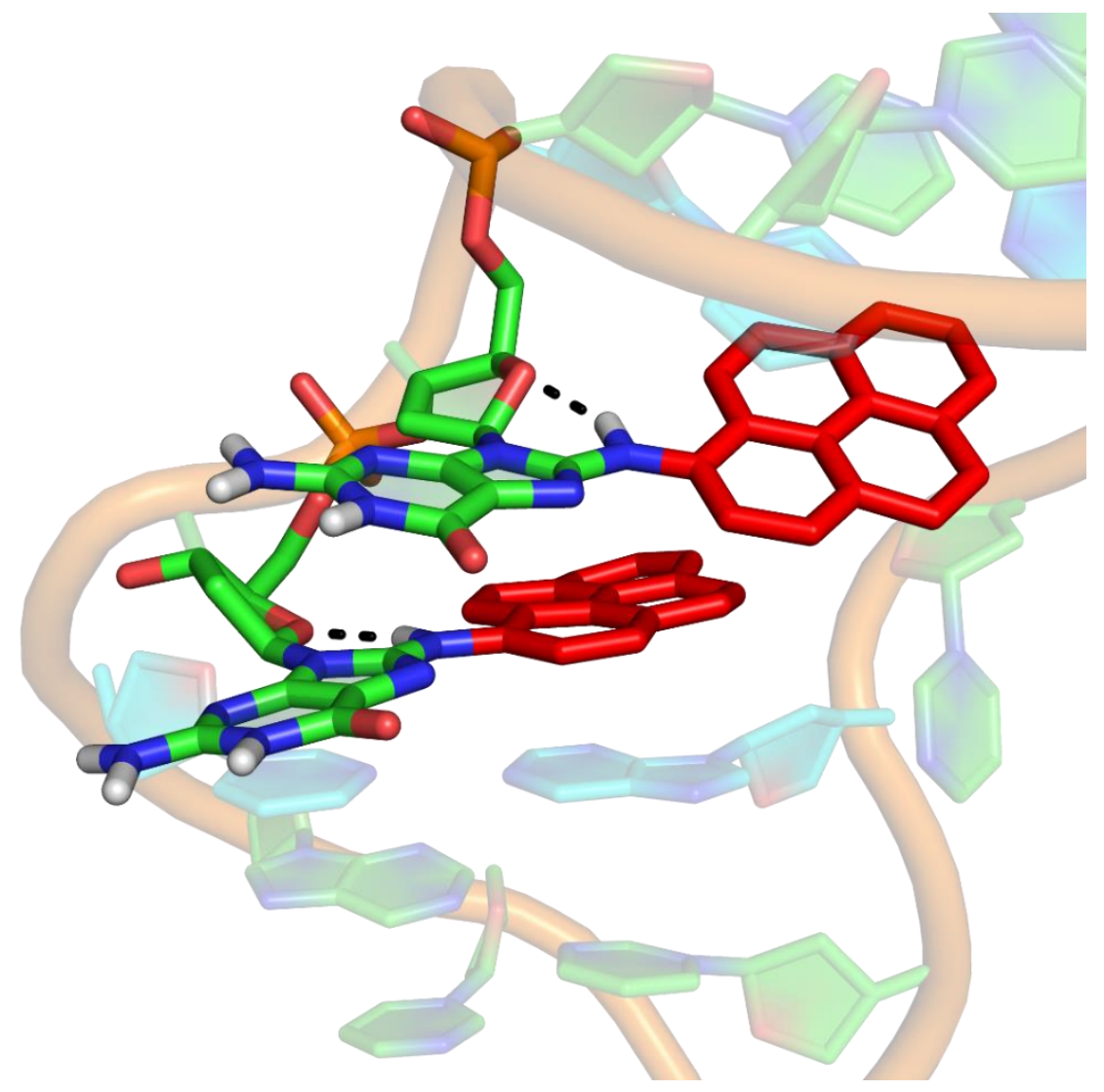

Figure S18: MD representative structure of ${ }^{A P} d G$ diadducted $D N A$ in the $G_{1}^{S} G_{2}^{S}$ conformation, highlighting the distorted sugar at $\mathrm{G}_{2}$ and $\mathrm{G}_{3}$, the intramolecular hydrogen bonds between the linker $\mathrm{N}-\mathrm{H}$ and $\mathrm{O} 4$ ', and the lack of lesion overlay due to helical twist. The adducted bases are shown in green and the bulky moiety in red. 
${ }^{A N} d G \quad A P d G$
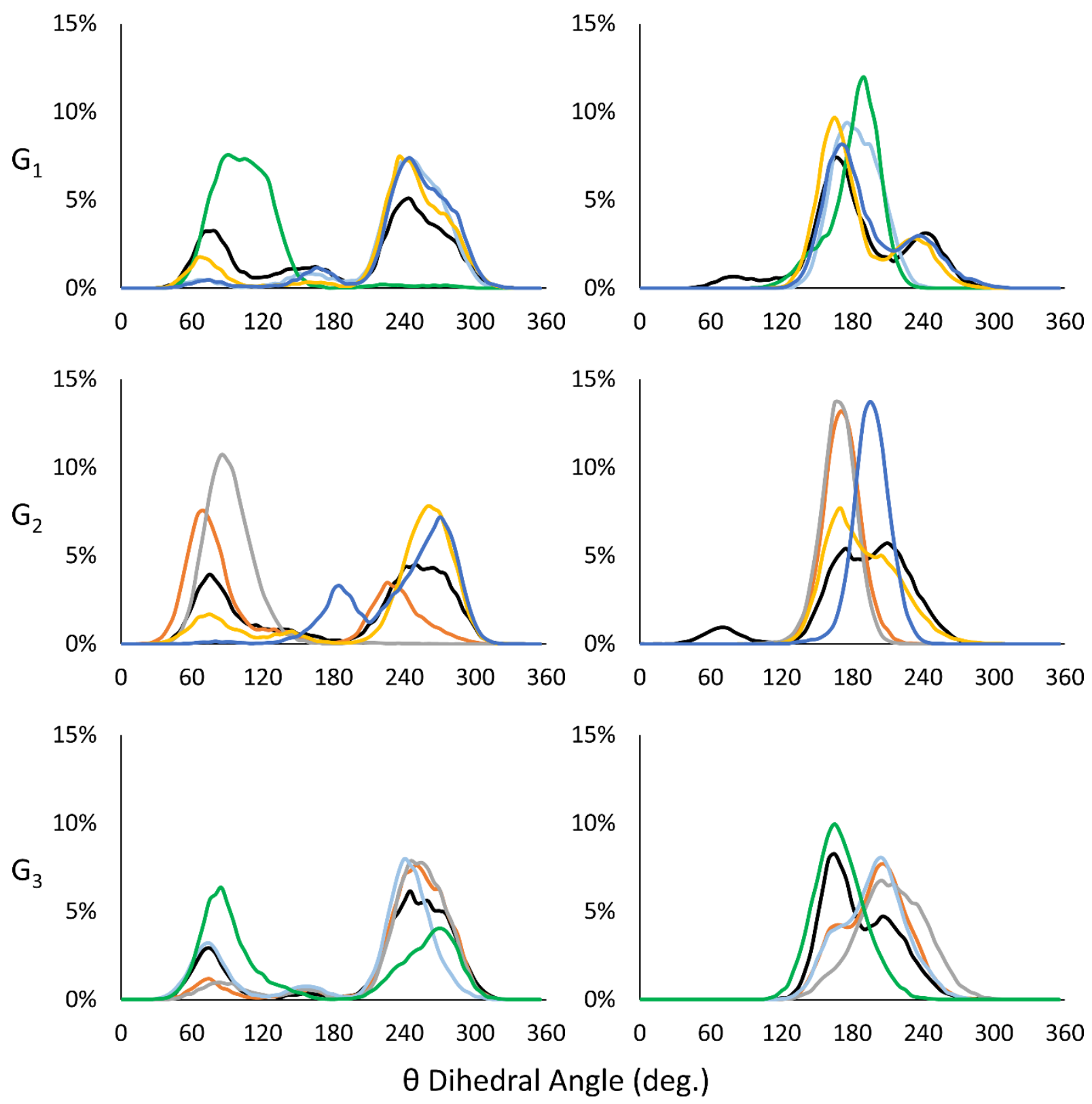

- None $\quad \mathrm{G}_{1}^{\mathrm{B}}=\mathrm{G}_{1}^{\mathrm{S}}=\mathrm{G}_{2}^{\mathrm{B}} \quad-\mathrm{G}_{2}^{\mathrm{S}} \quad \mathrm{G}_{3}^{\mathrm{B}} \quad-\mathrm{G}_{3}^{\mathrm{S}}$ Figure S19: Histograms of the $\theta$ dihedral angle over all MD replicas for ${ }^{A N} \mathrm{dG}$ (left) and ${ }^{A P} \mathrm{dG}$ (right) adducted DNA duplexes in the $\mathrm{S}$ conformation at $\mathrm{G}_{1}$ (top), $\mathrm{G}_{2}$ (middle) or $\mathrm{G}_{3}$ (bottom). The color indicates the presence and location of a second lesion, with 'None' corresponding to monoadducted DNA. 

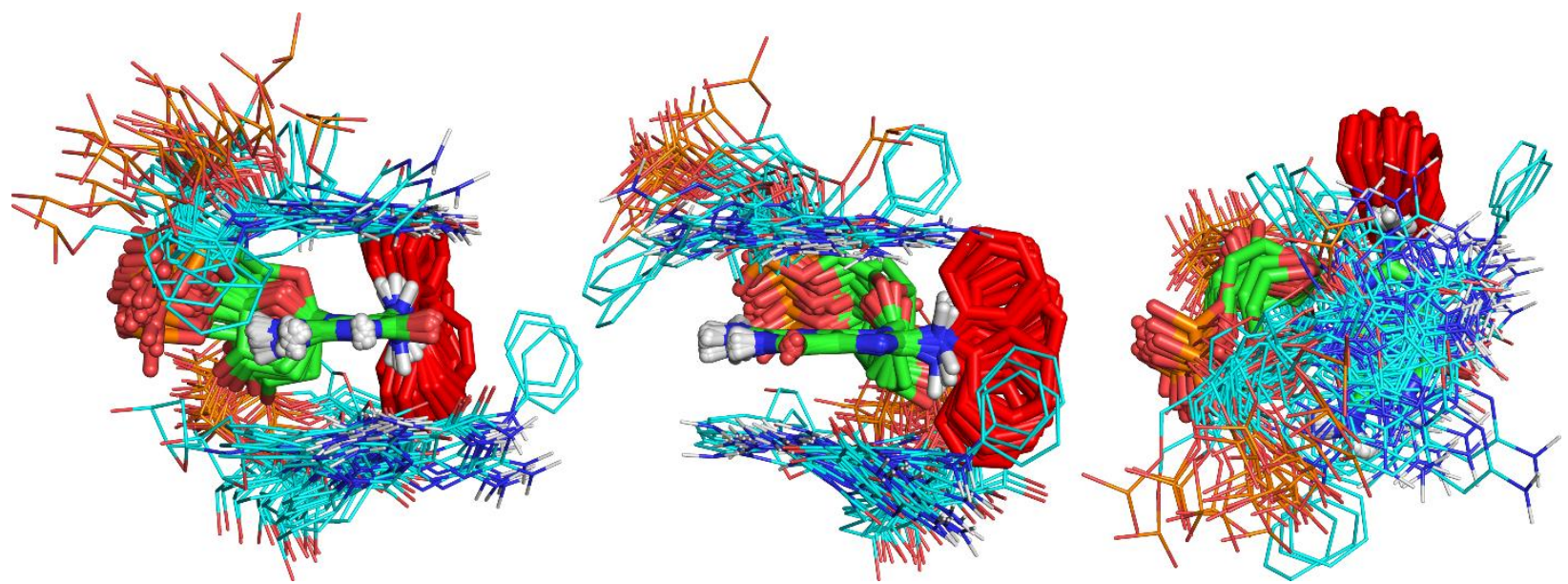

Figure S20: Different views of an overlay of the lesion site from representative structures obtained from each MD simulation of ${ }^{A N} \mathrm{dG}$ diadducted DNA in the $S$ conformation, highlighting the nonplanarity of the bulky group with respect to the nucleobase. The adducted base is shown in green, the bulky moiety in red, and the flanking bases in cyan.

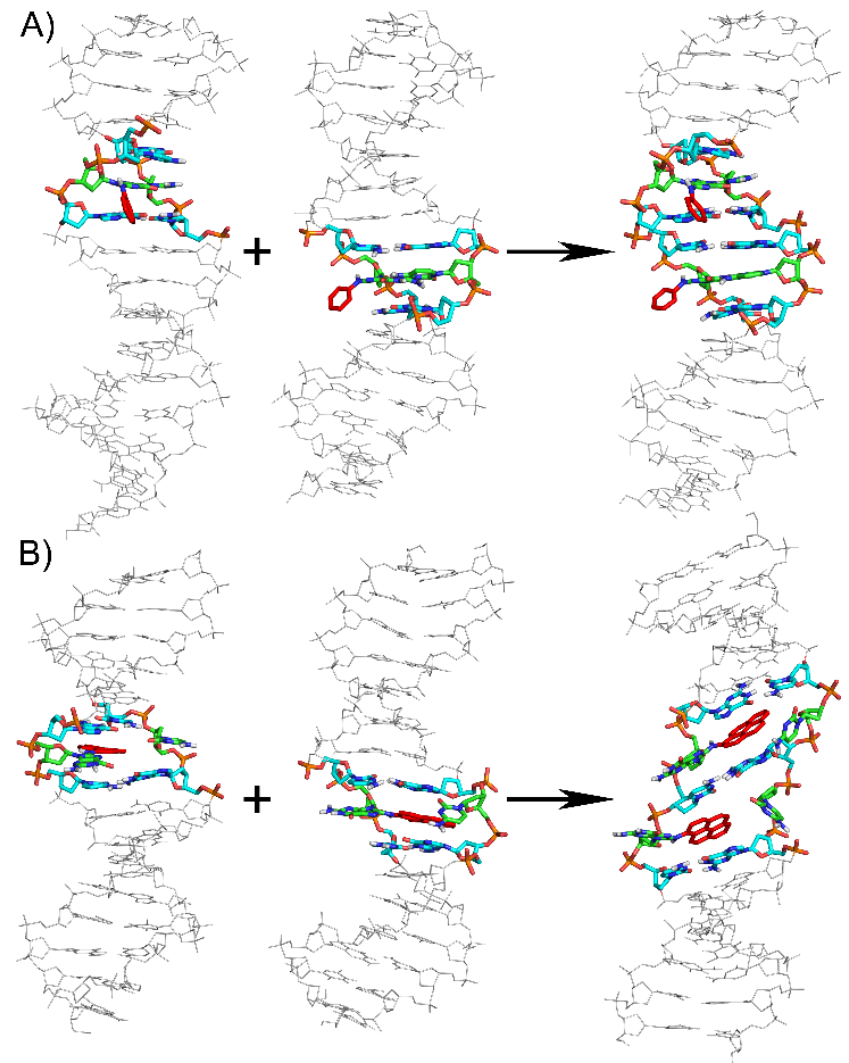

Figure S21: Structures of $A$ ) the $G_{1}^{B}$ (left), $G_{3}^{B}$ (middle) and $G_{1}^{B} G_{3}^{B}$ (right) ${ }^{A N} d G$-containing helices and $B$ ) the $\mathrm{G}_{2}^{S}$ (left), $\mathrm{G}_{3}^{S}$ (middle) and $\mathrm{G}_{2}^{S} \mathrm{G}_{3}^{S}$ (right) ${ }^{A P} d G$-containing helices, highlighting the range in helical distortion observed for monoadducted and diadducted DNA duplexes. 
Table S1: Average (standard deviation in parentheses) backbone RMSD ( $\AA$ ) across MD simulations of undamaged and damaged DNA. ${ }^{\mathrm{a}}$

\begin{tabular}{|c|c|c|c|c|c|c|c|c|}
\hline \multirow{2}{*}{ System } & \multirow{2}{*}{$\begin{array}{c}\text { Primary } \\
\text { Lesion }\end{array}$} & \multirow{2}{*}{$\begin{array}{c}\text { Secondary } \\
\text { Lesion }\end{array}$} & \multicolumn{3}{|c|}{${ }^{A N} \mathrm{dG}$} & \multicolumn{3}{|c|}{${ }^{A P} d G$} \\
\hline & & & Replica 1 & Replica 2 & Replica 3 & Replica 1 & Replica 2 & Replica 3 \\
\hline Undamaged & absent & absent & $2.9(0.7)$ & $2.9(0.7)$ & $3.0(0.7)$ & $2.9(0.7)$ & $2.9(0.7)$ & $3.0(0.7)$ \\
\hline \multirow{6}{*}{ Monoadducted } & $\mathrm{G}_{1}^{B}$ & absent & $4.4(0.9)$ & $4.1(0.8)$ & $4.3(0.8)$ & $4.4(0.8)$ & $4.2(0.8)$ & $4.2(0.8)$ \\
\hline & $\mathrm{G}_{1}^{\mathfrak{S}}$ & absent & $4.4(0.7)$ & $4.4(0.7)$ & $5.7(1.0)$ & $5.3(0.8)$ & $5.6(1.0)$ & $5.3(0.7)$ \\
\hline & $\mathrm{G}_{2}^{B}$ & absent & $4.2(0.8)$ & $4.1(0.8)$ & $4.2(0.8)$ & $4.5(0.8)$ & $4.2(0.8)$ & $4.6(0.7)$ \\
\hline & $\mathrm{G}_{2}^{S}$ & absent & $4.5(0.8)$ & $4.9(0.8)$ & $4.3(0.7)$ & $6.4(0.8)$ & $5.8(0.6)$ & $5.6(0.6)$ \\
\hline & $\mathrm{G}_{3}^{B}$ & absent & $3.1(0.6)$ & $3.5(0.8)$ & $3.2(0.6)$ & $3.2(0.6)$ & $3.4(0.7)$ & $3.2(0.7)$ \\
\hline & $\mathrm{G}_{3}^{S}$ & absent & $3.5(0.5)$ & $3.6(0.7)$ & $4.0(0.8)$ & $4.5(0.8)$ & $4.6(0.6)$ & $4.5(1.1)$ \\
\hline \multirow{12}{*}{ Diadducted } & $\mathrm{G}_{1}^{p}$ & $\mathrm{G}_{2}^{\mathrm{B}}$ & $2.6(0.6)$ & $2.7(0.6)$ & $2.7(0.6)$ & $3.2(0.7)$ & $3.3(0.7)$ & $3.7(0.7)$ \\
\hline & $\mathrm{G}_{1}^{B}$ & $\mathrm{G}\{$ & $3.5(0.7)$ & $4.0(0.5)$ & $4.1(0.5)$ & $3.6(0.7)$ & $3.9(0.7)$ & $3.4(0.7)$ \\
\hline & G1 & $\mathrm{G}^{B}$ & $2.9(0.5)$ & $3.0(0.6)$ & $3.2(0.6)$ & $3.8(0.7)$ & $3.7(0.7)$ & $4.0(0.6)$ \\
\hline & $\mathrm{G}_{1}^{\$}$ & $\bar{G}$ & $6.5(0.7)$ & $7.0(0.7)$ & $6.7(0.7)$ & $4.9(1.0)$ & $4.9(1.1)$ & $4.7(0.9)$ \\
\hline & $\mathrm{G}_{1}^{8}$ & $\mathrm{G}_{3}^{B}$ & $3.8(0.8)$ & $3.8(0.8)$ & $3.4(0.7)$ & $3.4(0.7)$ & $3.4(0.7)$ & $3.7(0.7)$ \\
\hline & $\mathrm{G}_{1}^{B}$ & $\mathrm{G}$ & $4.1(0.7)$ & $3.9(0.8)$ & $3.8(0.7)$ & $3.7(0.7)$ & $3.3(0.6)$ & $3.2(0.6)$ \\
\hline & $\mathrm{G}_{1}^{\mathfrak{S}}$ & $\mathrm{G}_{3}^{\mathrm{B}}$ & $3.7(0.7)$ & $3.9(0.7)$ & $4.0(0.7)$ & $3.7(0.8)$ & $3.6(0.8)$ & $3.4(0.7)$ \\
\hline & $\mathrm{G}_{1}^{\mathfrak{S}}$ & $\mathrm{G}_{3}^{\mathfrak{s}}$ & $4.2(0.8)$ & $4.2(0.7)$ & $4.1(0.7)$ & $5.3(0.8)$ & $4.9(0.8)$ & $5.2(0.7)$ \\
\hline & $\mathrm{G}^{B}$ & $\mathrm{G}_{3}^{B}$ & $3.5(0.6)$ & $3.1(0.6)$ & $3.2(0.6)$ & $3.8(0.8)$ & $3.7(0.8)$ & $3.9(0.8)$ \\
\hline & $\mathrm{G}_{2}^{B}$ & $\mathrm{G}_{3}^{\{}$ & $3.7(0.6)$ & $3.8(0.6)$ & $3.5(0.6)$ & $7.0(0.7)$ & $7.0(0.6)$ & $6.9(1.5)$ \\
\hline & $\mathrm{G}\{$ & $\mathrm{G}_{3}^{B}$ & $3.3(0.6)$ & $4.6(1.3)$ & $3.4(0.7)$ & $5.8(1.0)$ & $5.0(0.7)$ & $5.1(0.7)$ \\
\hline & $\mathrm{G}\{$ & $\mathrm{G}\{\widehat{\zeta}$ & $7.4(1.2)$ & $5.7(2.3)$ & $3.3(0.7)$ & $4.9(1.0)$ & $5.0(0.9)$ & $5.3(1.1)$ \\
\hline
\end{tabular}

${ }^{a}$ RMSDs calculated with respect to the first frame of the corresponding $500 \mathrm{~ns}$ MD production phase. 
Table S2: Occupancies (\%) of the Watson-Crick hydrogen bonds at the terminal ends of DNA helices. $^{\mathrm{a}}$

\begin{tabular}{|c|c|c|c|c|c|c|c|c|}
\hline \multirow{2}{*}{ System } & \multirow{2}{*}{ Adduct } & \multirow{2}{*}{ Site(s) } & \multicolumn{3}{|c|}{ 5'-terminal end } & \multicolumn{3}{|c|}{ 3'-terminal end } \\
\hline & & & Replica 1 & Replica 2 & Replica 3 & Replica 1 & Replica 2 & Replica 3 \\
\hline Undamaged & absent & N/A & 99.4 & 99.5 & 99.4 & 99.7 & 99.8 & 99.7 \\
\hline \multirow{12}{*}{ Monoadducted } & \multirow{3}{*}{${ }^{\mathrm{AN}} \mathrm{dG}$} & $\mathrm{G}_{1}^{\mathrm{B}}$ & 99.1 & 99.2 & 99.9 & 99.8 & 99.8 & 99.9 \\
\hline & & $\mathrm{G}_{2}^{\mathrm{B}}$ & 99.4 & 99.3 & 99.8 & 99.7 & 99.7 & 99.8 \\
\hline & & $\mathrm{G}_{3}^{\mathrm{B}}$ & 99.0 & 99.7 & 99.8 & 99.7 & 99.8 & 99.8 \\
\hline & \multirow{3}{*}{${ }^{A P} \mathrm{dG}$} & $\mathrm{G}_{1}^{\mathrm{B}}$ & 99.2 & 99.3 & 99.8 & 99.1 & 99.8 & 99.8 \\
\hline & & $\mathrm{G}_{2}^{B}$ & 99.1 & 99.2 & 99.8 & 99.7 & 99.7 & 99.8 \\
\hline & & $\mathrm{G}_{3}^{\mathrm{B}}$ & 99.1 & 99.2 & 99.7 & 99.7 & 99.7 & 99.7 \\
\hline & \multirow{3}{*}{${ }^{\mathrm{AN}} \mathrm{dG}$} & $\mathrm{G}_{1}^{S}$ & 99.1 & 99.3 & 99.7 & 99.7 & 99.8 & 99.7 \\
\hline & & $\mathrm{G}_{2}^{S}$ & 99.6 & 99.2 & 99.7 & 99.7 & 99.8 & 99.7 \\
\hline & & $\mathrm{G}^{\mathfrak{S}}$ & 99.7 & 99.2 & 99.1 & 99.8 & 99.8 & 99.1 \\
\hline & \multirow{3}{*}{${ }^{A P} \mathrm{dG}$} & $\mathrm{G}_{1}$ & 99.3 & 99.1 & 99.7 & 99.7 & 99.8 & 99.7 \\
\hline & & $\mathrm{G}\{$ & 99.2 & 99.0 & 99.7 & 99.8 & 99.8 & 99.7 \\
\hline & & $\mathrm{G}_{3}^{\mathfrak{S}}$ & 99.2 & 99.2 & 99.8 & 99.7 & 99.7 & 99.8 \\
\hline \multirow{24}{*}{ Diadducted } & \multirow{3}{*}{${ }^{\mathrm{AN}} \mathrm{dG}$} & $\mathrm{G}_{1}^{\mathrm{B}} \mathrm{G}_{2}^{\mathrm{B}}$ & 99.2 & 99.1 & 99.7 & 99.8 & 99.9 & 99.7 \\
\hline & & $\mathrm{G}_{1}^{\mathrm{B}} \mathrm{G}_{3}^{\mathrm{B}}$ & 99.1 & 99.1 & 99.7 & 99.8 & 99.8 & 99.7 \\
\hline & & $\mathrm{G}_{2}^{B} \mathrm{G}_{3}^{\mathrm{B}}$ & 99.3 & 99.3 & 99.8 & 99.7 & 99.8 & 99.8 \\
\hline & \multirow{3}{*}{${ }^{A P} \mathrm{dG}$} & $\mathrm{G}_{1}^{\mathrm{B}} \mathrm{G}_{2}^{\mathrm{B}}$ & 99.3 & 99.1 & 99.8 & 99.8 & 99.8 & 99.8 \\
\hline & & $\mathrm{G}_{1}^{\mathrm{B}} \mathrm{G}_{3}^{\mathrm{B}}$ & 99.3 & 99.2 & 99.7 & 99.7 & 99.7 & 99.7 \\
\hline & & $\mathrm{G}_{2}^{\mathrm{B}} \mathrm{G}_{3}^{\mathrm{B}}$ & 99.2 & 99.2 & 99.9 & 99.7 & 99.8 & 99.9 \\
\hline & \multirow{3}{*}{${ }^{\mathrm{AN}} \mathrm{dG}$} & $\mathrm{G}_{1}^{\mathrm{B}} \mathrm{G}_{2}^{\mathrm{S}}$ & 99.1 & 99.3 & 99.8 & 99.9 & 99.7 & 99.8 \\
\hline & & $\mathrm{GBG}^{\mathrm{B}}$ & 99.1 & 99.2 & 99.7 & 99.8 & 99.7 & 99.7 \\
\hline & & $\mathrm{GB}_{2}^{\mathrm{B}} \mathrm{s}$ & 99.3 & 99.4 & 99.8 & 99.8 & 99.8 & 99.8 \\
\hline & \multirow{3}{*}{${ }^{\mathrm{AP}} \mathrm{dG}$} & $\mathrm{G}_{1}^{\mathrm{B}} \mathrm{G}_{2}^{\mathrm{S}}$ & 99.1 & 99.1 & 99.8 & 99.8 & 99.8 & 99.8 \\
\hline & & $\mathrm{G}_{1}^{B} \mathrm{G}_{3}^{\mathrm{S}}$ & 99.2 & 99.1 & 99.7 & 99.7 & 99.7 & 99.7 \\
\hline & & $\mathrm{G}_{2}^{B} \mathrm{G}_{3}^{\mathfrak{s}}$ & 99.2 & 99.4 & 99.7 & 99.8 & 99.9 & 99.7 \\
\hline & \multirow{3}{*}{${ }^{\mathrm{AN}} \mathrm{dG}$} & $\mathrm{G}_{1} \mathrm{G}_{2}^{\mathrm{B}}$ & 99.3 & 99.2 & 99.8 & 99.7 & 99.8 & 99.8 \\
\hline & & $\mathrm{G}_{1} \mathrm{G}_{3}^{\mathrm{B}}$ & 99.2 & 99.1 & 99.7 & 99.7 & 99.7 & 99.7 \\
\hline & & $\mathrm{G}\left\{\mathrm{G}^{\mathrm{B}}\right.$ & 99.4 & 99.2 & 99.7 & 99.8 & 99.8 & 99.7 \\
\hline & \multirow{3}{*}{${ }^{A P} \mathrm{dG}$} & $\mathrm{G}_{1} \mathrm{G}_{2}^{\mathrm{B}}$ & 99.1 & 99.4 & 99.7 & 99.8 & 99.8 & 99.7 \\
\hline & & $\mathrm{G}_{1} \mathrm{G}_{3}^{\mathrm{B}}$ & 99.1 & 99.0 & 99.7 & 99.7 & 99.7 & 99.7 \\
\hline & & $\mathrm{G}_{2} \mathrm{G}_{3}^{\mathrm{B}}$ & 99.4 & 99.2 & 99.8 & 99.9 & 99.7 & 99.8 \\
\hline & \multirow{3}{*}{${ }^{\mathrm{AN}} \mathrm{dG}$} & $\mathrm{G}\left\{\mathrm{G}_{2}\right.$ & 99.2 & 99.1 & 99.8 & 99.8 & 99.8 & 99.8 \\
\hline & & $\mathrm{G}_{1} \mathrm{G}^{\mathrm{S}}$ & 99.1 & 99.1 & 99.8 & 99.7 & 99.7 & 99.8 \\
\hline & & $\mathrm{G}\{\mathrm{G}\}$ & 99.2 & 99.6 & 99.8 & 99.8 & 99.7 & 99.8 \\
\hline & \multirow{3}{*}{${ }^{\mathrm{AP}} \mathrm{dG}$} & $\mathrm{G}\{\mathrm{G}\{$ & 99.4 & 99.3 & 99.7 & 99.8 & 99.7 & 99.7 \\
\hline & & $\mathrm{G}_{1} \mathrm{G}_{3}$ & 99.0 & 99.3 & 99.8 & 99.7 & 99.7 & 99.8 \\
\hline & & $\mathrm{G}\left\{\mathrm{G}_{3}\right\}$ & 99.2 & 99.2 & 99.8 & 99.7 & 99.8 & 99.8 \\
\hline
\end{tabular}

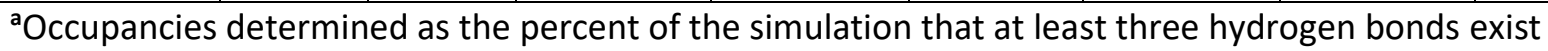
according to a heavy atom distance $<3.2 \AA$ and a heavy atom-hydrogen-heavy atom angle $>120^{\circ}$. 
Table S3: Occupancies (\%) of the Watson-Crick hydrogen bonds at the lesion site for undamaged and monoadducted DNA duplexes in the B conformation. ${ }^{a}$

\begin{tabular}{|c|c|c|c|c|c|c|c|}
\hline $\begin{array}{c}\text { Hydrogen } \\
\text { Bond }^{b}\end{array}$ & Adduct $^{c}$ & Site & Replica 1 & Replica 2 & Replica 3 & Average & $\begin{array}{l}\text { Standard } \\
\text { Deviation }\end{array}$ \\
\hline \multirow{9}{*}{$\begin{array}{l}(\mathrm{X}) \mathrm{N} 2-\mathrm{H} \\
\bullet \bullet \bullet \mathrm{O} 2(\mathrm{C})\end{array}$} & \multirow{3}{*}{ absent } & $\mathrm{G}_{1}$ & 99.9 & 99.9 & 99.9 & 99.9 & 0.0 \\
\hline & & $\mathrm{G}_{2}$ & 99.8 & 99.8 & 99.7 & 99.8 & 0.0 \\
\hline & & $\mathrm{G}_{3}$ & 99.9 & 100.0 & 99.9 & 99.9 & 0.0 \\
\hline & \multirow{3}{*}{${ }^{A N} \mathrm{dG}$} & $\mathrm{G}_{1}^{\mathrm{B}}$ & 99.8 & 99.9 & 99.9 & 99.9 & 0.0 \\
\hline & & $\mathrm{G}_{2}^{\mathrm{B}}$ & 99.9 & 99.8 & 99.9 & 99.9 & 0.0 \\
\hline & & $\mathrm{G}_{3}^{B}$ & 99.9 & 100.0 & 100.0 & 100.0 & 0.0 \\
\hline & \multirow{3}{*}{${ }^{A P} \mathrm{dG}$} & $\mathrm{G}_{1}^{\mathrm{B}}$ & 99.8 & 99.8 & 99.9 & 99.8 & 0.1 \\
\hline & & $\mathrm{G}_{2}^{\mathrm{B}}$ & 99.8 & 99.7 & 99.9 & 99.8 & 0.1 \\
\hline & & $\mathrm{G}_{3}^{\mathrm{B}}$ & 99.8 & 99.7 & 99.8 & 99.8 & 0.0 \\
\hline \multirow{9}{*}{$\begin{array}{l}(X) N 1-H \\
\bullet \bullet N 3(C)\end{array}$} & \multirow{3}{*}{ absent } & $\mathrm{G}_{1}$ & 100.0 & 99.9 & 100.0 & 100.0 & 0.0 \\
\hline & & $\mathrm{G}_{2}$ & 100.0 & 99.9 & 99.9 & 99.9 & 0.0 \\
\hline & & $\mathrm{G}_{3}$ & 100.0 & 100.0 & 100.0 & 100.0 & 0.0 \\
\hline & \multirow{3}{*}{${ }^{A N} \mathrm{dG}$} & $\mathrm{G}_{1}^{\mathrm{B}}$ & 98.6 & 99.3 & 99.3 & 99.1 & 0.3 \\
\hline & & $\mathrm{G}_{2}^{\mathrm{B}}$ & 99.9 & 99.7 & 99.8 & 99.8 & 0.1 \\
\hline & & $\mathrm{G}_{3}^{\mathrm{B}}$ & 99.7 & 99.8 & 99.8 & 99.8 & 0.0 \\
\hline & \multirow{3}{*}{${ }^{A P} d G$} & $\mathrm{G}_{1}^{\mathrm{B}}$ & 99.9 & 99.6 & 99.9 & 99.8 & 0.1 \\
\hline & & $\mathrm{G}_{2}^{\mathrm{B}}$ & 99.9 & 100.0 & 100.0 & 100.0 & 0.0 \\
\hline & & $\mathrm{G}_{3}^{\mathrm{B}}$ & 99.9 & 99.7 & 99.9 & 99.8 & 0.1 \\
\hline \multirow{9}{*}{$\begin{array}{l}\text { (C)N4-H } \\
\bullet \bullet \bullet O 6(X)\end{array}$} & \multirow{3}{*}{ absent } & $\mathrm{G}_{1}$ & 98.9 & 98.7 & 99.1 & 98.9 & 0.1 \\
\hline & & $\mathrm{G}_{2}$ & 98.8 & 98.8 & 98.8 & 98.8 & 0.0 \\
\hline & & $\mathrm{G}_{3}$ & 99.3 & 99.1 & 99.1 & 99.2 & 0.1 \\
\hline & \multirow{3}{*}{${ }^{A N} \mathrm{dG}$} & $\mathrm{G}_{1}^{\mathrm{B}}$ & 97.0 & 97.6 & 97.7 & 97.4 & 0.3 \\
\hline & & $\mathrm{G}_{2}^{\mathrm{B}}$ & 98.8 & 98.5 & 98.5 & 98.6 & 0.2 \\
\hline & & $\mathrm{G}_{3}^{B}$ & 98.3 & 98.2 & 98.4 & 98.3 & 0.1 \\
\hline & \multirow{3}{*}{${ }^{A P} \mathrm{dG}$} & $\mathrm{G}_{1}^{B}$ & 97.6 & 97.5 & 97.7 & 97.6 & 0.1 \\
\hline & & $\mathrm{G}_{2}^{\mathrm{B}}$ & 99.3 & 99.2 & 99.3 & 99.2 & 0.1 \\
\hline & & $\mathrm{G}_{3}^{\mathrm{B}}$ & 98.5 & 98.3 & 98.5 & 98.5 & 0.1 \\
\hline
\end{tabular}

${ }^{a}$ Occupancies determined as the percent of the simulation that a hydrogen bonds exist according to a heavy atom distance $<3.2 \AA$ and a heavy atom-hydrogen-heavy atom angle $>120^{\circ}$. ${ }^{\text {b } X}$ represents the damaged nucleotide for adducted DNA or the undamaged nucleotide for natural DNA. 'Absent indicates undamaged DNA. ${ }^{\mathrm{d}}$ Standard deviation calculated over the occupancies from the three replicates. 
Table S4: Occupancies (\%) of the Watson-Crick hydrogen bonds at the lesion sites for diadducted DNA duplexes in the B conformation. ${ }^{\mathrm{a}}$

\begin{tabular}{|c|c|c|c|c|c|c|c|c|}
\hline \multirow{2}{*}{$\begin{array}{l}\text { Hydrogen } \\
\text { Bond }^{b}\end{array}$} & \multirow{2}{*}{ Adduct } & \multirow{2}{*}{$\begin{array}{l}\text { Primary } \\
\text { Lesion }\end{array}$} & \multicolumn{6}{|c|}{ Secondary Lesion } \\
\hline & & & $\mathrm{G}_{1}-\mathrm{B}$ & $\mathrm{G}_{1}-\mathrm{S}$ & $\mathrm{G}_{2}-\mathrm{B}$ & $\mathrm{G}_{2}-\mathrm{S}$ & $\mathrm{G}_{3}-\mathrm{B}$ & $\mathrm{G}_{3}-\mathrm{S}$ \\
\hline \multirow{6}{*}{$(\mathrm{X}) \mathrm{N} 2-\mathrm{H} \bullet \bullet \cdot \mathrm{O} 2(\mathrm{C})$} & \multirow{3}{*}{${ }^{A N} \mathrm{dG}$} & $\mathrm{G}_{1}^{\mathrm{B}}$ & $\mathrm{N} / \mathrm{A}$ & $\mathrm{N} / \mathrm{A}$ & 99.9 & 95.3 & 99.9 & 99.7 \\
\hline & & $\mathrm{G}_{2}^{\mathrm{B}}$ & 99.8 & 99.7 & N/A & N/A & 99.8 & 99.8 \\
\hline & & $\mathrm{G}_{3}^{B}$ & 99.9 & 99.9 & 99.9 & 99.8 & N/A & $\mathrm{N} / \mathrm{A}$ \\
\hline & \multirow{3}{*}{${ }^{A P} \mathrm{dG}$} & $\mathrm{G}_{1}^{\mathrm{B}}$ & $\mathrm{N} / \mathrm{A}$ & $\mathrm{N} / \mathrm{A}$ & 99.8 & 97.8 & 99.9 & 99.9 \\
\hline & & $\mathrm{G}_{2}^{\mathrm{B}}$ & 99.8 & 99.3 & N/A & $\mathrm{N} / \mathrm{A}$ & 99.8 & 99.8 \\
\hline & & $\mathrm{G}_{3}^{B}$ & 99.9 & 99.8 & 99.8 & 99.4 & $\mathrm{~N} / \mathrm{A}$ & $\mathrm{N} / \mathrm{A}$ \\
\hline \multirow{6}{*}{$(\mathrm{X}) \mathrm{N} 1-\mathrm{H} \bullet \bullet \mathrm{N} 3(\mathrm{C})$} & \multirow{3}{*}{${ }^{\mathrm{AN}} \mathrm{dG}$} & $\mathrm{G}_{1}^{\mathrm{B}}$ & $\mathrm{N} / \mathrm{A}$ & $\mathrm{N} / \mathrm{A}$ & 99.5 & 95.5 & 99.1 & 98.4 \\
\hline & & $\mathrm{G}_{2}^{\mathrm{B}}$ & 99.9 & 99.5 & $\mathrm{~N} / \mathrm{A}$ & $\mathrm{N} / \mathrm{A}$ & 99.8 & 99.9 \\
\hline & & $\mathrm{G}_{3}^{B}$ & 99.7 & 99.2 & 99.8 & 99.6 & $\mathrm{~N} / \mathrm{A}$ & $\mathrm{N} / \mathrm{A}$ \\
\hline & \multirow{3}{*}{${ }^{A P} \mathrm{dG}$} & $\mathrm{G}_{1}^{\mathrm{B}}$ & $\mathrm{N} / \mathrm{A}$ & $\mathrm{N} / \mathrm{A}$ & 99.1 & 94.9 & 99.7 & 99.6 \\
\hline & & $\mathrm{G}_{2}^{\mathrm{B}}$ & 99.9 & 99.5 & $\mathrm{~N} / \mathrm{A}$ & $\mathrm{N} / \mathrm{A}$ & 99.9 & 100.0 \\
\hline & & $\mathrm{G}_{3}^{B}$ & 99.8 & 99.9 & 99.9 & 99.9 & $\mathrm{~N} / \mathrm{A}$ & $N / A$ \\
\hline \multirow{6}{*}{ 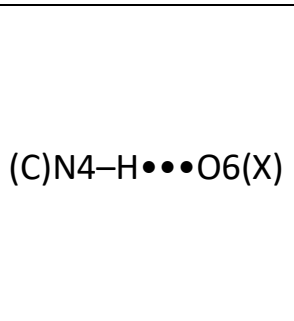 } & \multirow{3}{*}{${ }^{\mathrm{AN}} \mathrm{dG}$} & $\mathrm{G}_{1}^{B}$ & $\mathrm{~N} / \mathrm{A}$ & $\mathrm{N} / \mathrm{A}$ & 97.4 & 82.6 & 97.2 & 96.3 \\
\hline & & $\mathrm{G}_{2}^{B}$ & 98.9 & 97.6 & $\mathrm{~N} / \mathrm{A}$ & $\mathrm{N} / \mathrm{A}$ & 98.6 & 98.8 \\
\hline & & $\mathrm{G}_{3}^{B}$ & 98.1 & 97.5 & 98.1 & 98.1 & $\mathrm{~N} / \mathrm{A}$ & $\mathrm{N} / \mathrm{A}$ \\
\hline & \multirow{3}{*}{${ }^{A P} \mathrm{dG}$} & $\mathrm{G}_{1}^{B}$ & $\mathrm{~N} / \mathrm{A}$ & $\mathrm{N} / \mathrm{A}$ & 95.6 & 90.8 & 97.1 & 97.6 \\
\hline & & $\mathrm{G}_{2}^{\mathrm{B}}$ & 99.5 & 98.7 & N/A & $\mathrm{N} / \mathrm{A}$ & 99.0 & 99.3 \\
\hline & & $\mathrm{G}_{3}^{\mathrm{B}}$ & 98.4 & 98.5 & 98.5 & 98.6 & $N / A$ & $\mathrm{~N} / \mathrm{A}$ \\
\hline
\end{tabular}

accupancies determined as the percent of the simulation that a hydrogen bonds exist according to a heavy atom distance $<3.2 \AA$ and a heavy atom-hydrogen-heavy atom angle $>120^{\circ}$. ${ }^{b} \mathrm{X}$ represents the damaged nucleotide. 
Table S5: Occupancies (\%) of the Watson-Crick hydrogen bonds flanking the nucleotides at the lesion site in monoadducted DNA duplexes. ${ }^{\mathrm{a}, \mathrm{b}}$

\begin{tabular}{|c|c|c|c|c|c|c|c|}
\hline \multirow{2}{*}{ Adduct } & \multirow{2}{*}{ Site } & Replica 1 & Replica 2 & Replica 3 & Replica 1 & Replica 2 & Replica 3 \\
\hline & & \multicolumn{3}{|c|}{$\mathrm{dC5}: \mathrm{dG} 28$} & \multicolumn{3}{|c|}{$\mathrm{dG} 7: \mathrm{dC} 26$} \\
\hline \multirow{2}{*}{${ }^{A N} d G$} & $\mathrm{G}_{1}^{B}$ & 99.1 & 98.9 & 99.1 & 98.8 & 98.7 & 98.7 \\
\hline & $\mathrm{G}_{1}^{\mathrm{S}}$ & 99.0 & 98.9 & 97.4 & 99.2 & 98.9 & 98.1 \\
\hline \multirow{3}{*}{${ }^{A P} \mathrm{dG}$} & $\mathrm{G}_{1}^{\mathrm{B}}$ & 98.7 & 98.7 & 98.8 & 98.9 & 98.8 & 98.8 \\
\hline & $\mathrm{G}_{1}^{\mathrm{S}}$ & 98.1 & 98.4 & 98.8 & 99.1 & 98.9 & 99.1 \\
\hline & & \multicolumn{3}{|c|}{ dG6:dC27 } & \multicolumn{3}{|c|}{ dC8:dG25 } \\
\hline \multirow{2}{*}{${ }^{A N} \mathrm{dG}$} & $\mathrm{G}_{2}^{\mathrm{B}}$ & 99.1 & 98.9 & 99.0 & 99.4 & 99.1 & 99.3 \\
\hline & $\mathrm{G}_{2}^{\mathrm{S}}$ & 98.5 & 96.0 & 98.2 & 97.8 & 98.4 & 97.9 \\
\hline \multirow{3}{*}{${ }^{A P} \mathrm{dG}$} & $\mathrm{G}_{2}^{B}$ & 98.9 & 98.8 & 99.1 & 99.3 & 99.2 & 99.3 \\
\hline & $\mathrm{G}_{2}^{\mathrm{S}}$ & 97.8 & 98.5 & 98.5 & 95.0 & 98.1 & 92.9 \\
\hline & & \multicolumn{3}{|c|}{ dC8:dG25 } & \multicolumn{3}{|c|}{ dC10:dG23 } \\
\hline \multirow{2}{*}{${ }^{A N} \mathrm{dG}$} & $\mathrm{G}_{3}^{B}$ & 99.2 & 99.1 & 99.2 & 98.9 & 98.9 & 98.8 \\
\hline & $\mathrm{G}_{3}^{\mathrm{S}}$ & 99.4 & 98.7 & 99.2 & 97.0 & 97.5 & 92.3 \\
\hline \multirow{2}{*}{${ }^{A P} \mathrm{dG}$} & $\mathrm{G}_{3}^{B}$ & 99.1 & 98.7 & 98.9 & 98.8 & 98.7 & 99.0 \\
\hline & $\mathrm{G}_{3}^{S}$ & 98.8 & 98.2 & 98.5 & 93.0 & 92.2 & 96.1 \\
\hline
\end{tabular}

${ }^{a}$ Nucleotide numbering is illustrated in Figure $58 .{ }^{b}$ Occupancies determined as the percent of the simulation that at least three hydrogen bonds exist according to a heavy atom distance $<3.2 \AA$ and a heavy atom-hydrogen-heavy atom angle $>120^{\circ}$. 
Table S6: Occupancies (\%) of the Watson-Crick hydrogen bonds flanking the nucleotides at the lesion sites in $\mathrm{G}_{1} \mathrm{G}_{2}$ diadducted DNA duplexes. ${ }^{\mathrm{a}, \mathrm{b}}$

\begin{tabular}{|c|c|c|c|c|c|c|c|}
\hline \multirow{2}{*}{ Adduct } & \multirow{2}{*}{ Sites } & Replica 1 & Replica 2 & Replica 3 & Replica 1 & Replica 2 & Replica 3 \\
\cline { 2 - 8 } & & \multicolumn{3}{|c|}{$\mathrm{dC5}$ dG28 } & \multicolumn{3}{|c|}{$\mathrm{dC8}$ dG25 } \\
\hline \multirow{3}{*}{ AN dG } & $\mathrm{G}_{1}^{\mathrm{B}} \mathrm{G}_{2}^{\mathrm{B}}$ & 98.9 & 99.0 & 99.0 & 99.4 & 99.4 & 99.2 \\
\cline { 2 - 8 } & $\mathrm{G}_{1}^{\mathrm{G}} \mathrm{G}_{2}^{\mathrm{S}}$ & 99.4 & 99.3 & 99.4 & 96.7 & 97.3 & 97.6 \\
\cline { 2 - 8 } & $\mathrm{G}_{1}^{\mathrm{G}} \mathrm{G}_{2}^{\mathrm{B}}$ & 98.9 & 99.1 & 99.0 & 99.2 & 99.5 & 99.3 \\
\cline { 2 - 8 } & $\mathrm{G}_{1}^{\mathrm{S}} \mathrm{G}_{2}^{\mathrm{S}}$ & 97.7 & 98.4 & 98.6 & 98.3 & 97.6 & 97.7 \\
\hline \multirow{4}{*}{$\mathrm{AP} d \mathrm{dG}$} & $\mathrm{G}_{1}^{\mathrm{B}} \mathrm{G}_{2}^{\mathrm{B}}$ & 98.0 & 98.5 & 98.5 & 99.0 & 99.3 & 99.2 \\
\cline { 2 - 8 } & $\mathrm{G}_{1}^{\mathrm{B}} \mathrm{G}_{2}^{\mathrm{S}}$ & 97.8 & 98.6 & 98.6 & 99.3 & 99.3 & 99.2 \\
\cline { 2 - 8 } & $\mathrm{G}_{1}^{\mathrm{G}} \mathrm{G}_{2}^{\mathrm{B}}$ & 98.2 & 97.7 & 98.5 & 99.3 & 99.0 & 99.2 \\
\cline { 2 - 8 } & $\mathrm{G}_{1}^{\mathrm{S}} \mathrm{G}_{2}^{\mathrm{S}}$ & 98.4 & 98.4 & 98.2 & 99.3 & 99.4 & 99.2 \\
\hline
\end{tabular}

${ }^{a}$ Nucleotide numbering is illustrated in Figure $58 .{ }^{b}$ Occupancies determined as the percent of the simulation that at least three hydrogen bonds exist according to a heavy atom distance $<3.2 \AA$ and a heavy atom-hydrogen-heavy atom angle $>120^{\circ}$. 
Table S7: Occupancies (\%) of the Watson-Crick hydrogen bonds flanking the nucleotides at the lesion sites in $\mathrm{G}_{1} \mathrm{G}_{3}$ diadducted DNA duplexes. ${ }^{\mathrm{a}, \mathrm{b}}$

\begin{tabular}{|c|c|c|c|c|c|c|c|}
\hline \multirow{2}{*}{ Adduct } & \multirow{2}{*}{ Sites } & Replica 1 & Replica 2 & Replica 3 & Replica 1 & Replica 2 & Replica 3 \\
\hline & & \multicolumn{3}{|c|}{ dC5:dG28 } & \multicolumn{3}{|c|}{$\mathrm{dG7}: \mathrm{dC26}$} \\
\hline \multirow{4}{*}{${ }^{A N} \mathrm{dG}$} & $\mathrm{G}_{1}^{\mathrm{B}} \mathrm{G}_{3}^{\mathrm{B}}$ & 99.0 & 99.2 & 99.1 & 98.8 & 99.0 & 98.6 \\
\hline & $\mathrm{G}_{1}^{\mathrm{B}} \mathrm{G}_{3}^{\mathrm{S}}$ & 99.0 & 99.1 & 99.1 & 99.0 & 99.0 & 98.8 \\
\hline & $\mathrm{G}_{1}^{S} \mathrm{G}_{3}^{\mathrm{B}}$ & 99.0 & 98.9 & 98.7 & 98.7 & 99.1 & 99.2 \\
\hline & $\mathrm{G}_{1}^{S} \mathrm{G}_{3}^{S}$ & 98.6 & 98.8 & 99.1 & 99.1 & 99.5 & 99.3 \\
\hline \multirow{4}{*}{${ }^{A P} \mathrm{dG}$} & $\mathrm{G}_{1}^{\mathrm{B}} \mathrm{G}_{3}^{\mathrm{B}}$ & 98.7 & 98.8 & 98.7 & 99.0 & 99.2 & 98.9 \\
\hline & $\mathrm{G}_{1}^{\mathrm{B}} \mathrm{G}_{3}^{\mathrm{S}}$ & 98.8 & 98.6 & 98.8 & 99.0 & 99.0 & 98.9 \\
\hline & $\mathrm{G}_{1}^{S} \mathrm{G}_{3}^{\mathrm{B}}$ & 98.5 & 98.5 & 98.1 & 99.2 & 99.1 & 99.1 \\
\hline & $\mathrm{G}_{1}^{S} \mathrm{G}_{3}^{S}$ & 98.5 & 97.2 & 98.4 & 99.1 & 99.3 & 99.1 \\
\hline & & \multicolumn{3}{|c|}{$\mathrm{dC} 8: \mathrm{dG} 25$} & \multicolumn{3}{|c|}{$\mathrm{dC} 10: \mathrm{dG} 23$} \\
\hline \multirow{4}{*}{${ }^{A N} \mathrm{dG}$} & $\mathrm{G}_{1}^{\mathrm{B}} \mathrm{G}_{3}^{\mathrm{B}}$ & 99.0 & 99.2 & 99.0 & 98.7 & 98.9 & 98.7 \\
\hline & $\mathrm{G}_{1}^{\mathrm{B}} \mathrm{G}_{3}^{\mathrm{S}}$ & 99.2 & 99.1 & 99.2 & 93.5 & 94.8 & 98.9 \\
\hline & $\mathrm{G}_{1}^{\mathrm{S}} \mathrm{G}_{3}^{\mathrm{B}}$ & 99.0 & 99.2 & 99.0 & 98.7 & 98.7 & 98.9 \\
\hline & $\mathrm{G}_{1}^{S} \mathrm{G}_{3}^{S}$ & 99.0 & 99.3 & 99.1 & 95.6 & 98.4 & 94.2 \\
\hline \multirow{4}{*}{${ }^{A P} \mathrm{dG}$} & $\mathrm{G}_{1}^{\mathrm{B}} \mathrm{G}_{3}^{\mathrm{B}}$ & 98.7 & 98.9 & 98.7 & 98.7 & 98.9 & 98.9 \\
\hline & $\mathrm{G}_{1}^{\mathrm{B}} \mathrm{G}_{3}^{\mathrm{S}}$ & 98.8 & 99.1 & 99.1 & 93.7 & 93.0 & 92.6 \\
\hline & $\mathrm{G}_{1}^{S} \mathrm{G}_{3}^{\mathrm{B}}$ & 98.7 & 98.7 & 98.3 & 98.8 & 98.7 & 98.9 \\
\hline & $\mathrm{G}_{1}^{S} \mathrm{G}_{3}^{S}$ & 99.1 & 99.0 & 99.1 & 95.4 & 94.5 & 93.7 \\
\hline
\end{tabular}

${ }^{a}$ Nucleotide numbering is illustrated in Figure S8. ${ }^{b}$ Occupancies determined as the percent of the simulation that at least three hydrogen bonds exist according to a heavy atom distance $<3.2 \AA$ and a heavy atom-hydrogen-heavy atom angle $>120^{\circ}$. 
Table S8: Occupancies (\%) of the Watson-Crick hydrogen bonds flanking the nucleotides at the lesion sites in $\mathrm{G}_{2} \mathrm{G}_{3}$ diadducted DNA duplexes. ${ }^{\mathrm{a}, \mathrm{b}}$

\begin{tabular}{|c|c|c|c|c|}
\hline \multirow{2}{*}{ Adduct } & \multirow{2}{*}{ Sites } & Replica 1 & Replica 2 & Replica 3 \\
\hline & & \multicolumn{3}{|c|}{ dG6:dC27 } \\
\hline \multirow{4}{*}{${ }^{A N} d G$} & $\mathrm{G}_{2}^{\mathrm{B}} \mathrm{G}_{3}^{\mathrm{B}}$ & 98.9 & 98.8 & 99.1 \\
\hline & $\mathrm{G}_{2}^{\mathrm{B}} \mathrm{G}_{3}^{\mathrm{S}}$ & 99.0 & 99.1 & 99.0 \\
\hline & $\mathrm{G}_{2}^{\mathrm{S}} \mathrm{G}_{3}^{\mathrm{B}}$ & 99.0 & 97.7 & 98.7 \\
\hline & $\mathrm{G}_{2}^{S} \mathrm{G}_{3}^{\mathrm{S}}$ & 98.8 & 98.9 & 98.9 \\
\hline \multirow{4}{*}{${ }^{A P} d G$} & $\mathrm{G}_{2}^{\mathrm{B}} \mathrm{G}_{3}^{\mathrm{B}}$ & 98.8 & 98.8 & 98.7 \\
\hline & $\mathrm{G}_{2}^{B} \mathrm{G}_{3}^{\mathrm{S}}$ & 98.9 & 98.9 & 99.1 \\
\hline & $\mathrm{G}_{2}^{\mathrm{S}} \mathrm{G}_{3}^{\mathrm{B}}$ & 97.2 & 97.8 & 97.9 \\
\hline & $\mathrm{G}_{2}^{S} \mathrm{G}_{3}^{S}$ & 99.4 & 99.3 & 99.3 \\
\hline & & \multicolumn{3}{|c|}{ dC8:dG25 } \\
\hline \multirow{4}{*}{${ }^{\mathrm{AN}} \mathrm{dG}$} & $\mathrm{G}_{2}^{\mathrm{B}} \mathrm{G}_{3}^{\mathrm{B}}$ & 99.1 & 99.2 & 99.2 \\
\hline & $\mathrm{G}_{2}^{\mathrm{B}} \mathrm{G}_{3}^{\mathrm{S}}$ & 99.1 & 98.2 & 98.9 \\
\hline & $\mathrm{G}_{2}^{S} \mathrm{G}_{3}^{\mathrm{B}}$ & 97.6 & 97.8 & 97.8 \\
\hline & $\mathrm{G}_{2}^{S} \mathrm{G}_{3}^{S}$ & 94.0 & 98.9 & 98.6 \\
\hline \multirow{4}{*}{${ }^{A P} d G$} & $\mathrm{G}_{2}^{\mathrm{B}} \mathrm{G}_{3}^{\mathrm{B}}$ & 99.0 & 98.9 & 99.0 \\
\hline & $\mathrm{G}_{2}^{\mathrm{B}} \mathrm{G}_{3}^{\mathrm{S}}$ & 99.2 & 99.0 & 98.9 \\
\hline & $\mathrm{G}_{2}^{\mathrm{S}} \mathrm{G}_{3}^{\mathrm{B}}$ & 46.2 & 94.2 & 92.1 \\
\hline & $\mathrm{G}_{2}^{\mathrm{S}} \mathrm{G}_{3}^{\mathrm{S}}$ & 77.1 & 75.4 & 41.8 \\
\hline & & \multicolumn{3}{|c|}{ dC10:dG23 } \\
\hline \multirow{4}{*}{${ }^{A N} d G$} & $\mathrm{G}_{2}^{\mathrm{B}} \mathrm{G}_{3}^{\mathrm{B}}$ & 98.7 & 98.9 & 98.9 \\
\hline & $\mathrm{G}_{2}^{\mathrm{B}} \mathrm{G}_{3}^{\mathrm{S}}$ & 93.5 & 95.6 & 94.7 \\
\hline & $\mathrm{G}_{2}^{\mathrm{S}} \mathrm{G}_{3}^{\mathrm{B}}$ & 99.0 & 98.9 & 99.0 \\
\hline & $\mathrm{G}_{2}^{\mathrm{S}} \mathrm{G}_{3}^{\mathrm{S}}$ & 98.7 & 97.6 & 99.3 \\
\hline \multirow{4}{*}{${ }^{A P} d G$} & $\mathrm{G}_{2}^{\mathrm{B}} \mathrm{G}_{3}^{\mathrm{B}}$ & 98.9 & 98.6 & 98.7 \\
\hline & $\mathrm{G}_{2}^{\mathrm{B}} \mathrm{G}_{3}^{\mathrm{S}}$ & 93.8 & 94.7 & 95.9 \\
\hline & $\mathrm{G}_{2}^{\mathrm{S}} \mathrm{G}_{3}^{\mathrm{B}}$ & 99.0 & 99.0 & 99.0 \\
\hline & $\mathrm{G}_{2}^{S} \mathrm{G}_{3}^{S}$ & 99.1 & 99.0 & 98.7 \\
\hline
\end{tabular}

${ }^{a}$ Nucleotide numbering is illustrated in Figure $\mathrm{S} 8 .{ }^{\mathrm{b}}$ Occupancies determined as the percent of the simulation that at least three hydrogen bonds exist according to a heavy atom distance $<3.2 \AA$ and a heavy atom-hydrogen-heavy atom angle $>120^{\circ}$. 
Table S9: Average (standard deviation in parentheses) $\chi$ dihedral angle (deg.) for each replica and across all replicas $\left(\chi^{\text {avg }}\right)$ for undamaged and monoadducted DNA. ${ }^{a}$

\begin{tabular}{|c|c|c|c|c|c|c|}
\hline Adduct & Site & Replica 1 & Replica 2 & Replica 3 & $\chi^{\text {avg }}$ & $\mathrm{BSE}^{\mathrm{C}}$ \\
\hline \multirow{3}{*}{ absent $^{b}$} & $\mathrm{G}_{1}$ & $256.2(19.3)$ & 252.9 (18.9) & 252.5 (19.4) & $253.9(2.0)$ & 1.2 \\
\hline & $\mathrm{G}_{2}$ & 244.1 (16.7) & 241.1 (19.7) & $244.3(15.2)$ & $243.2(1.8)$ & 0.9 \\
\hline & $\mathrm{G}_{3}$ & $254.1(16.2)$ & $254.1(15.8)$ & $254.5(15.3)$ & $254.2(0.2)$ & 0.4 \\
\hline \multirow{3}{*}{${ }^{A N} \mathrm{dG}$} & $\mathrm{G}_{1}^{\mathrm{B}}$ & $231.0(12.8)$ & $225.5(17.7)$ & $229.9(14.2)$ & $228.8(2.9)$ & 0.7 \\
\hline & $\mathrm{G}_{2}^{\mathrm{B}}$ & $225.0(13.1)$ & $223.4(14.6)$ & $225.9(12.2)$ & $224.8(1.3)$ & 0.6 \\
\hline & $\mathrm{G}_{3}^{\mathrm{B}}$ & $230.7(12.5)$ & $231.0(12.2)$ & $231.2(12.3)$ & $231.0(0.3)$ & 0.1 \\
\hline \multirow{3}{*}{${ }^{A P} \mathrm{dG}$} & $\mathrm{G}_{1}^{\mathrm{B}}$ & $196.0(16.3)$ & $210.9(21.6)$ & $201.2(19.7)$ & $202.7(7.5)$ & 13.3 \\
\hline & $\mathrm{G}_{2}^{B}$ & $215.9(16.6)$ & $217.6(13.8)$ & $221.0(12.3)$ & $218.1(2.6)$ & 0.8 \\
\hline & $\mathrm{G}_{3}^{B}$ & $216.5(21.8)$ & $217.9(21.9)$ & $218.7(21.1)$ & $217.7(1.1)$ & 3.1 \\
\hline \multirow{3}{*}{${ }^{A N} \mathrm{dG}$} & $\mathrm{G}_{1}^{\mathrm{S}}$ & $55.3(12.8)$ & $59.5(16.3)$ & $58.2(14.4)$ & $57.6(2.2)$ & 0.6 \\
\hline & $\mathrm{G}_{2}^{\mathrm{S}}$ & $48.4(16.3)$ & $57.4(16.8)$ & $51.7(17.6)$ & $52.5(4.6)$ & 0.9 \\
\hline & $\mathrm{G}_{3}^{S}$ & $50.3(21.9)$ & $54.7(18.8)$ & 44.5 (14.9) & $49.8(5.2)$ & 1.2 \\
\hline \multirow{3}{*}{${ }^{A P} d G$} & $\mathrm{G}_{1}^{\mathrm{S}}$ & $41.6(16.9)$ & $45.2(28.1)$ & $44.1(20.2)$ & 43.6 (1.9) & 0.9 \\
\hline & $\mathrm{G}_{2}^{S}$ & $55.9(38.7)$ & $51.9(46.4)$ & $40.8(19.9)$ & $49.6(7.8)$ & 3.0 \\
\hline & $\mathrm{G}_{3}^{S}$ & $38.2(16.6)$ & 39.4 (14.9) & $47.4(54.2)$ & $41.7(5.0)$ & 4.8 \\
\hline
\end{tabular}

${ }^{\mathrm{a}} \chi=\angle\left(\mathrm{O}^{\prime}-\mathrm{C} 1^{\prime}-\mathrm{N} 9-\mathrm{C} 4\right)$. See Figure 1 in the main text. ${ }^{\mathrm{b}}$ Undamaged DNA. 'Block standard error (BSE) extrapolated from all frames of the three replicas fitted to a monomolecular growth model.

Table S10: Average (standard deviation in parentheses) $\theta$ dihedral angle (deg.) for each replica and across all replicas $\left(\theta^{\text {avg }}\right)$ for monoadducted DNA. ${ }^{\text {a }}$

\begin{tabular}{|c|c|c|c|c|c|c|}
\hline Adduct & Site & Replica 1 & Replica 2 & Replica 3 & $\theta^{\text {avg }}$ & BSE $^{\text {b }}$ \\
\hline \multirow{3}{*}{ AN dG } & $\mathrm{G}_{1}^{\text {B }}$ & $124.9(22.7)$ & $132.6(31.6)$ & $126.8(25.2)$ & $128.1(4.0)$ & 1.7 \\
\cline { 2 - 7 } & $\mathrm{G}_{2}^{B}$ & $141.8(23.5)$ & $143.9(25.1)$ & $141.2(22.9)$ & $142.3(1.4)$ & 2.1 \\
\cline { 2 - 7 } & $\mathrm{G}_{3}^{\mathrm{B}}$ & $132.7(19.6)$ & $132.6(18.6)$ & $132.5(19.1)$ & $132.6(0.1)$ & 0.2 \\
\hline \multirow{4}{*}{ APdG } & $\mathrm{G}_{1}^{\mathrm{B}}$ & $182.8(41.3)$ & $171.6(38.7)$ & $177.0(41.3)$ & $177.1(5.6)$ & 18.9 \\
\cline { 2 - 7 } & $\mathrm{G}_{2}^{\mathrm{B}}$ & $173.1(22.0)$ & $172.3(19.3)$ & $169.9(20.7)$ & $171.8(1.7)$ & 11.5 \\
\cline { 2 - 7 } & $\mathrm{G}_{3}^{\mathrm{B}}$ & $158.1(33.4)$ & $157.8(33.5)$ & $155.6(33.4)$ & $157.2(1.4)$ & 11.5 \\
\hline \multirow{3}{*}{ AN dG } & $\mathrm{G}_{1}^{\mathrm{S}}$ & $263.0(40.2)$ & $241.9(26.0)$ & $235.6(58.0)$ & $246.8(14.4)$ & 11.4 \\
\cline { 2 - 7 } & $\mathrm{G}_{2}^{\mathrm{S}}$ & $244.9(77.7)$ & $99.1(85.7)$ & $245.4(69.7)$ & $196.5(84.4)$ & 10.5 \\
\cline { 2 - 7 } & $\mathrm{G}_{3}^{\mathrm{S}}$ & $262.9(27.0)$ & $79.3(86.9)$ & $246.2(22.4)$ & $196.1(101.6)$ & 8.5 \\
\hline \multirow{3}{*}{ AP dG } & $\mathrm{G}_{2}^{\mathrm{S}}$ & $171.9(21.2)$ & $163.3(49.1)$ & $223.6(33.8)$ & $186.3(32.6)$ & 16.6 \\
\cline { 2 - 7 } & $\mathrm{G}_{2}^{\mathrm{S}}$ & $182.3(58.1)$ & $197.3(29.9)$ & $202.5(28.7)$ & $194.1(10.5)$ & 13.0 \\
\cline { 2 - 7 } & $\mathrm{G}_{3}^{\mathrm{S}}$ & $195.3(30.6)$ & $181.8(28.0)$ & $186.6(28.1)$ & $187.9(6.8)$ & 18.2 \\
\hline
\end{tabular}

${ }^{\mathrm{a}} \theta=\angle$ (N9-C8-N10-C10). See Figure 1 in the main text. ${ }^{\mathrm{b}}$ Block standard error (BSE) extrapolated from all frames of the three replicas fitted to a monomolecular growth model. 
Table S11: Average (standard deviation in parentheses) $\phi$ dihedral angle (deg.) for each replica and across all replicas $\left(\phi^{\text {avg }}\right)$ for monoadducted DNA. ${ }^{a}$

\begin{tabular}{|c|c|c|c|c|c|c|}
\hline Adduct & Site & Replica 1 & Replica 2 & Replica 3 & $\phi^{\text {avg }}$ & BSE $^{\text {b }}$ \\
\hline \multirow{4}{*}{ AN dG } & $\mathrm{G}_{1}^{\mathrm{B}}$ & $0.6(11.8)$ & $0.4(11.9)$ & $0.6(11.8)$ & $0.5(0.1)$ & 0.2 \\
\cline { 2 - 7 } & $\mathrm{G}_{2}^{\mathrm{B}}$ & $6.0(12.3)$ & $6.0(12.4)$ & $6.3(12.3)$ & $6.1(0.1)$ & 0.2 \\
\cline { 2 - 7 } & $\mathrm{G}_{3}^{\mathrm{B}}$ & $2.7(11.9)$ & $2.5(11.8)$ & $2.8(11.9)$ & $2.7(0.1)$ & 0.1 \\
\hline \multirow{3}{*}{ AP dG } & $\mathrm{G}_{1}^{\mathrm{B}}$ & $4.3(12.3)$ & $7.3(13.2)$ & $5.0(12.6)$ & $5.5(1.3)$ & 0.4 \\
\cline { 2 - 7 } & $\mathrm{G}_{2}^{\mathrm{B}}$ & $16.7(12.1)$ & $17.8(11.7)$ & $17.5(11.6)$ & $17.3(0.4)$ & 0.2 \\
\cline { 2 - 7 } & $\mathrm{G}_{3}^{\mathrm{B}}$ & $10.8(13.0)$ & $11.0(12.9)$ & $10.8(13.0)$ & $10.9(0.1)$ & 0.5 \\
\hline \multirow{3}{*}{$\mathrm{AN} \mathrm{dG}$} & $\mathrm{G}_{1}^{\mathrm{S}}$ & $3.3(11.1)$ & $2.7(10.9)$ & $2.8(10.8)$ & $2.9(0.2)$ & 0.2 \\
\cline { 2 - 7 } & $\mathrm{G}_{2}^{\mathrm{S}}$ & $-0.5(10.6)$ & $0.4(10.7)$ & $-0.1(10.9)$ & $-0.1(0.3)$ & 0.1 \\
\cline { 2 - 7 } & $\mathrm{G}_{3}^{\mathrm{S}}$ & $1.4(10.7)$ & $0.0(9.9)$ & $1.5(9.4)$ & $1.0(0.7)$ & 15.7 \\
\hline \multirow{3}{*}{$\mathrm{AP} \mathrm{dG}$} & $\mathrm{G}_{1}^{\mathrm{S}}$ & $-3.8(11.2)$ & $-4.0(11.3)$ & $-3.3(10.4)$ & $-3.7(0.3)$ & 0.1 \\
\cline { 2 - 7 } & $\mathrm{G}_{2}^{\mathrm{S}}$ & $-4.8(12.3)$ & $-6.1(12.1)$ & $-7.8(11.6)$ & $-6.3(1.2)$ & 0.3 \\
\cline { 2 - 7 } & $\mathrm{G}_{3}^{\mathrm{S}}$ & $-7.3(11.1)$ & $-7.5(11.0)$ & $-6.6(11.7)$ & $-7.2(0.4)$ & 0.3 \\
\hline
\end{tabular}

${ }^{\mathrm{a}} \phi=\angle(\mathrm{C} 8-\mathrm{N} 10-\mathrm{C} 10-\mathrm{C} 11)$. See Figure 1 in the main text. ${ }^{\mathrm{b}}$ Block standard error (BSE) extrapolated from all frames of the three replicas fitted to a monomolecular growth model. 
Table S12: Occupancies (\%) of the non-Watson-Crick hydrogen bonds at the lesion site of monoadducted DNA. ${ }^{a, b}$

\begin{tabular}{|c|c|c|c|c|c|c|c|}
\hline Adduct & Site & Hydrogen Bond ${ }^{c}$ & $\begin{array}{c}\text { Replica } \\
1\end{array}$ & $\begin{array}{c}\text { Replica } \\
2\end{array}$ & $\begin{array}{c}\text { Replica } \\
3\end{array}$ & Average $^{d}$ & $\begin{array}{c}\text { Standard } \\
\text { Deviation }^{\text {e }}\end{array}$ \\
\hline \multirow{4}{*}{${ }^{A N} d G$} & $\mathrm{G}_{1}^{\mathrm{B}}$ & $(\mathrm{X} 6) \mathrm{N} 10-\mathrm{H} \bullet \bullet \mathrm{O}^{\prime}(\mathrm{C} 5)$ & 33.0 & 25.8 & 29.9 & 29.6 & 2.9 \\
\hline & $\mathrm{G}_{1}^{B}$ & $(\mathrm{X} 6) \mathrm{N} 10-\mathrm{H} \bullet \bullet \mathrm{O}^{\prime}(\mathrm{X} 6)$ & 48.5 & 52.2 & 50.3 & 50.3 & 1.5 \\
\hline & $\mathrm{G}_{2}^{\mathrm{B}}$ & $(\mathrm{X} 7) \mathrm{N} 10-\mathrm{H} \bullet \bullet \mathrm{O}^{\prime}(\mathrm{X} 7)$ & 80.2 & 79.9 & 79.5 & 79.9 & 0.3 \\
\hline & $\mathrm{G}_{3}^{B}$ & $(\mathrm{X9}) \mathrm{N} 10-\mathrm{H} \bullet \bullet \mathrm{O}^{\prime}(\mathrm{X9})$ & 75.1 & 75.2 & 74.6 & 75.0 & 0.3 \\
\hline \multirow{3}{*}{${ }^{A P} \mathrm{dG}$} & $\mathrm{G}_{1}^{B}$ & $(\mathrm{X} 6) \mathrm{N} 10-\mathrm{H} \bullet \bullet \mathrm{O}^{\prime}(\mathrm{X} 6)$ & 53.5 & 50.1 & 53.7 & 52.4 & 1.6 \\
\hline & $\mathrm{G}_{2}^{B}$ & $(\mathrm{X} 7) \mathrm{N} 10-\mathrm{H} \bullet \bullet \mathrm{O}^{\prime}(\mathrm{X} 7)$ & 48.5 & 48.8 & 46.3 & 47.9 & 1.1 \\
\hline & $\mathrm{G}_{3}^{B}$ & $(\mathrm{X9}) \mathrm{N} 10-\mathrm{H} \bullet \bullet \mathrm{O}^{\prime}(\mathrm{X9})$ & 60.7 & 60.7 & 59.9 & 60.4 & 0.4 \\
\hline \multirow{14}{*}{${ }^{A N} d G$} & $\mathrm{G}_{1}^{\mathrm{S}}$ & $(\mathrm{C} 27) \mathrm{N} 4-\mathrm{H} \bullet \bullet \cdot \mathrm{O} 6(\mathrm{X} 6)$ & 63.5 & 0.0 & 0.0 & 21.2 & 29.9 \\
\hline & $\mathrm{G}_{1}^{\mathrm{S}}$ & $(\mathrm{X} 6) \mathrm{N} 10-\mathrm{H} \bullet \bullet \cdot \mathrm{N} 1(\mathrm{G} 28)$ & 19.4 & 21.3 & 0.0 & 13.5 & 9.6 \\
\hline & $\mathrm{G}_{1}^{\mathrm{S}}$ & $(\mathrm{X} 6) \mathrm{N} 10-\mathrm{H} \bullet \bullet \cdot \mathrm{N} 2(\mathrm{G} 28)$ & 45.9 & 50.7 & 0.0 & 32.2 & 22.9 \\
\hline & $\mathrm{G}_{1}^{\mathrm{S}}$ & $(\mathrm{X} 6) \mathrm{N} 10-\mathrm{H} \bullet \bullet \bullet 4^{\prime}(\mathrm{G} 7)$ & 6.1 & 0.0 & 46.9 & 17.7 & 20.8 \\
\hline & $\mathrm{G}_{1}^{\mathrm{S}}$ & $(\mathrm{C} 27) \mathrm{N} 4-\mathrm{H} \bullet \bullet \mathrm{OP}(\mathrm{C} 26)$ & 5.7 & 8.0 & 10.1 & 7.9 & 1.8 \\
\hline & $\mathrm{G}_{2}^{\mathrm{S}}$ & $(\mathrm{C} 26) \mathrm{N} 4-\mathrm{H} \bullet \bullet \bullet \mathrm{O}(\mathrm{X} 7)$ & 18.8 & 57.5 & 28.6 & 35.0 & 16.4 \\
\hline & $\mathrm{G}_{2}^{S}$ & $(\mathrm{C} 26) \mathrm{N} 4-\mathrm{H} \bullet \bullet \mathrm{N} 7(\mathrm{X} 7)$ & 7.9 & 22.9 & 15.5 & 15.4 & 6.1 \\
\hline & $\mathrm{G}_{2}^{\mathrm{S}}$ & $(\mathrm{X} 7) \mathrm{N} 10-\mathrm{H} \bullet \bullet \bullet \mathrm{O} 2(\mathrm{C} 27)$ & 17.6 & 5.7 & 12.6 & 11.9 & 13.5 \\
\hline & $\mathrm{G}_{2}^{S}$ & $(\mathrm{X} 7) \mathrm{N} 10-\mathrm{H} \bullet \bullet \bullet 4^{\prime}(\mathrm{C} 8)$ & 17.3 & 34.1 & 10.4 & 20.6 & 7.1 \\
\hline & $\mathrm{G}_{2}^{S}$ & $(\mathrm{X} 7) \mathrm{N} 10-\mathrm{H} \bullet \bullet \cdot \mathrm{N} 2(\mathrm{G} 6)$ & 28.2 & 13.0 & 28.1 & 23.1 & 16.1 \\
\hline & $\mathrm{G}_{3}^{5}$ & $(\mathrm{C} 24) \mathrm{N} 4-\mathrm{H} \bullet \bullet \bullet O 6(X 9)$ & 0.0 & 37.1 & 0.0 & 12.4 & 17.5 \\
\hline & $\mathrm{G}_{3}^{S}$ & (X9)N10-H・••O4'(C10) & 0.0 & 33.8 & 0.0 & 11.3 & 15.9 \\
\hline & $\mathrm{G}_{3}^{\mathrm{S}}$ & $(\mathrm{X} 9) \mathrm{N} 10-\mathrm{H} \bullet \bullet \cdot \mathrm{N} 2(\mathrm{G} 25)$ & 37.1 & 12.7 & 50.8 & 33.5 & 15.7 \\
\hline & $\mathrm{G}_{3}^{S}$ & $(X 9) N 10-H \bullet \bullet N 3(G 25)$ & 37.6 & 21.9 & 21.0 & 26.8 & 7.6 \\
\hline \multirow{7}{*}{${ }^{A P} \mathrm{dG}$} & $\mathrm{G}_{1}^{\mathrm{S}}$ & $(\mathrm{C} 27) \mathrm{N} 4-\mathrm{H} \bullet \bullet O \mathrm{OP} 2(\mathrm{C} 26)$ & 60.7 & 0.0 & 33.0 & 31.3 & 24.8 \\
\hline & $\mathrm{G}_{1}^{\mathrm{S}}$ & $(\mathrm{C} 26) \mathrm{N} 4-\mathrm{H} \bullet \bullet \bullet \mathrm{O} 6(\mathrm{X} 6)$ & 0.0 & 0.0 & 29.7 & 9.9 & 14.0 \\
\hline & $\mathrm{G}_{1}^{\mathrm{S}}$ & $(\mathrm{X} 6) \mathrm{N} 10-\mathrm{H} \bullet \bullet \cdot \mathrm{N} 2(\mathrm{G} 28)$ & 0.0 & 6.1 & 36.4 & 14.1 & 15.9 \\
\hline & $\mathrm{G}_{2}^{\mathrm{S}}$ & $(\mathrm{X} 7) \mathrm{N} 10-\mathrm{H} \bullet \bullet \mathrm{N} 2(\mathrm{G} 6)$ & 7.3 & 8.6 & 10.1 & 8.7 & 1.2 \\
\hline & $\mathrm{G}_{2}^{S}$ & $(\mathrm{X} 7) \mathrm{N} 10-\mathrm{H} \bullet \bullet \mathrm{O}^{\prime}(\mathrm{X} 7)$ & 11.5 & 16.0 & 0.0 & 9.2 & 6.8 \\
\hline & $\mathrm{G}_{2}^{\mathrm{S}}$ & $(\mathrm{C} 26) \mathrm{N} 4-\mathrm{H} \bullet \bullet \bullet \mathrm{OP} 2(\mathrm{G} 25)$ & 19.8 & 34.1 & 23.3 & 25.7 & 6.1 \\
\hline & $\mathrm{G}_{3}^{S}$ & $(\mathrm{C} 24) \mathrm{N} 4-\mathrm{H} \bullet \bullet \bullet \mathrm{OP} 2(\mathrm{G} 23)$ & 30.5 & 43.6 & 36.1 & 36.7 & 5.4 \\
\hline
\end{tabular}

${ }^{a}$ Nucleotide numbering is illustrated in Figure $\mathrm{S} 8 .{ }^{\mathrm{b}}$ Occupancies determined as the percent of the simulation that a hydrogen bonds exist according to a heavy atom distance $<3.2 \AA$ and a heavy atomhydrogen-heavy atom angle $>120^{\circ} .{ }^{c} X$ represents the damaged nucleotide. ${ }^{\text {d Average occupancy }}$

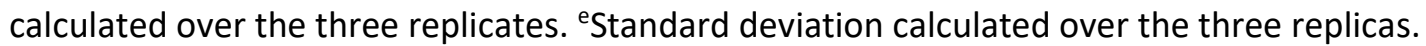


Table S13: Average (standard deviation in parentheses) lesion site interaction energy $(\mathrm{kJ} / \mathrm{mol})$ for undamaged and monoadducted DNA duplexes.

\begin{tabular}{|c|c|c|c|c|c|c|c|c|}
\hline Adduct & Site & LSIE $^{\text {avg, b }}$ & $\begin{array}{c}\text { Dataset } \\
\text { Standard } \\
\text { Deviation }^{c}\end{array}$ & $\begin{array}{l}\text { LSIE } \\
\text { BSE }^{d}\end{array}$ & $\begin{array}{l}\text { Average } \\
\text { Stacking }\end{array}$ & $\begin{array}{c}\text { Dataset } \\
\text { Standard } \\
\text { Deviation }\end{array}$ & $\begin{array}{l}\text { Average } \\
\text { Hydrogen } \\
\text { Bonding }^{f}\end{array}$ & $\begin{array}{c}\text { Dataset } \\
\text { Standard } \\
\text { Deviation }\end{array}$ \\
\hline \multirow{3}{*}{ absent $^{\mathrm{a}}$} & $\mathrm{G}_{1}$ & $-215.9(0.7)$ & 13.7 & 0.1 & $-97.3(0.6)$ & 12.1 & $-118.6(0.0)$ & 7.4 \\
\hline & $\mathrm{G}_{2}$ & $-208.4(0.1)$ & 14.8 & 0.1 & $-90.7(0.3)$ & 12.3 & $-117.7(0.1)$ & 7.6 \\
\hline & $\mathrm{G}_{3}$ & $-235.7(0.5)$ & 14.4 & 0.0 & $-117.2(0.4)$ & 12.3 & $-118.6(0.1)$ & 7.3 \\
\hline \multirow{3}{*}{${ }^{A N} \mathrm{dG}$} & $\mathrm{G}_{1}^{\mathrm{B}}$ & $-213.9(0.4)$ & 15.8 & 0.2 & $-104.5(0.5)$ & 12.9 & $-109.4(0.2)$ & 8.7 \\
\hline & $\mathrm{G}_{2}^{\mathrm{B}}$ & $-209.9(0.2)$ & 15.2 & 0.0 & $-99.8(0.2)$ & 12.9 & $-110.2(0.1)$ & 7.3 \\
\hline & $\mathrm{G}_{3}^{\mathrm{B}}$ & $-239.4(0.2)$ & 14.6 & 0.1 & $-129.6(0.1)$ & 12.7 & $-109.8(0.1)$ & 7.5 \\
\hline \multirow{3}{*}{${ }^{A P} \mathrm{dG}$} & $\mathrm{G}_{1}^{\mathrm{B}}$ & $-201.5(0.7)$ & 15.5 & 2.6 & $-96.7(0.4)$ & 13.9 & $-104.7(0.4)$ & 8.0 \\
\hline & $\mathrm{G}_{2}^{\mathrm{B}}$ & $-226.3(0.3)$ & 17.1 & 0.2 & $-119.2(0.4)$ & 14.8 & $-107.1(0.3)$ & 6.9 \\
\hline & $\mathrm{G}_{3}^{\mathrm{B}}$ & $-246.4(0.4)$ & 16.8 & 0.9 & $-140.5(0.3)$ & 14.5 & $-105.9(0.1)$ & 7.7 \\
\hline \multirow{3}{*}{${ }^{A N} \mathrm{dG}$} & $\mathrm{G}_{1}^{\mathrm{S}}$ & $-24.1(65.1)$ & 74.9 & 1.7 & $-40.0(35.8)$ & 44.4 & $15.9(29.3)$ & 33.5 \\
\hline & $\mathrm{G}_{2}^{S}$ & $-33.7(25.1)$ & 72.0 & 1.5 & $-48.7(15.0)$ & 46.2 & $15.1(10.1)$ & 30.1 \\
\hline & $\mathrm{G}_{3}^{S}$ & $-39.1(64.2)$ & 91.3 & 2.1 & $-47.9(39.7)$ & 57.5 & $8.9(24.6)$ & 36.5 \\
\hline \multirow{3}{*}{${ }^{A P} \mathrm{dG}$} & $\mathrm{G}_{1}^{\mathrm{S}}$ & $-123.3(36.2)$ & 51.6 & 1.1 & $-103.4(21.6)$ & 33.7 & $-19.9(15.1)$ & 23.8 \\
\hline & $\mathrm{G}_{2}^{S}$ & $-101.2(7.4)$ & 49.6 & 0.8 & $-106.8(4.4)$ & 37.6 & $5.6(3.6)$ & 16.1 \\
\hline & $\mathrm{G}_{3}^{S}$ & $-81.5(7.1)$ & 49.4 & 1.3 & $-86.7(4.9)$ & 37.8 & $5.2(2.3)$ & 13.9 \\
\hline
\end{tabular}

a Undamaged DNA. ${ }^{\mathrm{b}}$ Average of the sum of stacking and hydrogen-bonding interactions for the adducted base, bulky moiety and opposing $\mathrm{C}$, with standard deviation calculated between the replicas. 'Standard deviation calculated over the $1.5 \mu$ s dataset from the three replicas. ${ }^{\mathrm{d} B}$ Block standard error (BSE) extrapolated from all frames of the three replicas fitted to a monomolecular growth model. ${ }^{\mathrm{e}}$ Average of the stacking between the adduct (base and bulky moiety) plus the opposing $C$ and the flanking nucleobases, with standard deviation calculated between the replicas. ${ }^{\mathrm{f}}$ Average of the hydrogenbonding interaction between the adduct and the pairing $\mathrm{C}$, with standard deviation calculated between the replicas. 
Table S14: Average (standard deviation in parentheses) pseudostep parameters ${ }^{\mathrm{a}}$ for undamaged and monoadducted DNA.

\begin{tabular}{|c|c|c|c|c|c|c|c|c|}
\hline Adduct & Site & Rise $(\AA ̊)$ & Shift (Å) & Slide (Å) & Roll (deg.) & Tilt (deg.) & Twist (deg.) & $\begin{array}{c}\text { Axial Bend } \\
\text { (deg.) }\end{array}$ \\
\hline absent ${ }^{b}$ & N/A & $22.7(0.0)$ & $0.5(0.1)$ & $3.0(0.1)$ & $-9.7(0.5)$ & $0.2(0.7)$ & $-117.0(0.6)$ & $39.4(0.4)$ \\
\hline \multirow{3}{*}{${ }^{A N} \mathrm{dG}$} & $\mathrm{G}_{1}^{B}$ & $22.6(0.0)$ & $1.5(0.1)$ & $3.2(0.1)$ & $-8.5(0.4)$ & $-5.3(0.8)$ & $-119.9(0.6)$ & $39.1(0.3)$ \\
\hline & $\mathrm{G}_{2}^{\mathrm{B}}$ & $22.5(0.0)$ & $1.1(0.2)$ & $4.3(0.0)$ & $-12.7(0.1)$ & $-5.5(0.4)$ & $-121.8(0.1)$ & $39.3(0.4)$ \\
\hline & $\mathrm{G}_{3}^{\mathrm{B}}$ & $22.4(0.3)$ & $0.0(0.1)$ & $3.5(0.3)$ & $-16.3(2.0)$ & $-0.5(1.8)$ & $-117.4(2.5)$ & $39.0(0.8)$ \\
\hline \multirow{3}{*}{${ }^{A P} \mathrm{dG}$} & $\mathrm{G}_{1}^{\text {B }}$ & $22.4(0.0)$ & $2.3(0.1)$ & $4.2(0.1)$ & $-7.3(1.4)$ & $-6.2(1.0)$ & $-126.6(2.0)$ & $46.5(0.1)$ \\
\hline & $\mathrm{G}_{2}^{B}$ & $22.6(0.2)$ & $0.9(0.2)$ & $4.1(0.6)$ & $-13.3(1.5)$ & $-4.2(2.2)$ & $-120.8(3.4)$ & $37.6(2.2)$ \\
\hline & $\mathrm{G}_{3}^{\mathrm{B}}$ & $22.7(0.0)$ & $-0.5(0.0)$ & $3.4(0.1)$ & $-14.7(0.6)$ & $-2.2(0.1)$ & $-121.6(0.3)$ & $39.5(0.2)$ \\
\hline \multirow{3}{*}{${ }^{A N} \mathrm{dG}$} & $\mathrm{G}_{1}^{\mathrm{S}}$ & $21.5(1.2)$ & $2.4(1.7)$ & $6.0(3.1)$ & $-28.1(14.1)$ & $-22.7(11.1)$ & $-124.8(5.1)$ & $49.0(5.3)$ \\
\hline & $\mathrm{G}_{2}^{\mathrm{S}}$ & $22.5(0.1)$ & $0.7(0.1)$ & $3.7(0.5)$ & $-28.5(5.1)$ & $-3.4(2.3)$ & $-117.3(0.7)$ & $45.1(0.4)$ \\
\hline & $\mathrm{G}_{3}^{S}$ & $22.6(1.4)$ & $-0.8(1.4)$ & $4.5(3.0)$ & $-20.6(7.3)$ & 14.9 (11.6) & $-119.8(6.8)$ & 44.4 (7.3) \\
\hline \multirow{3}{*}{${ }^{A P} \mathrm{dG}$} & $\mathrm{G}_{2}^{\mathrm{S}}$ & $22.4(0.2)$ & $2.9(0.5)$ & $4.8(0.3)$ & $-24.5(5.6)$ & $-13.8(3.2)$ & $-129.9(4.5)$ & $44.1(2.5)$ \\
\hline & $\mathrm{G}_{2}^{\mathrm{S}}$ & $21.8(0.4)$ & $1.5(0.3)$ & $6.1(1.1)$ & $-31.6(3.9)$ & $-5.7(1.2)$ & $-131.6(2.5)$ & $46.8(0.8)$ \\
\hline & $\mathrm{G}_{3}^{S}$ & $21.9(0.4)$ & $-2.5(0.8)$ & $6.7(1.0)$ & $-21.6(2.3)$ & 14.5 (1.4) & $-134.0(1.0)$ & $46.7(1.2)$ \\
\hline
\end{tabular}

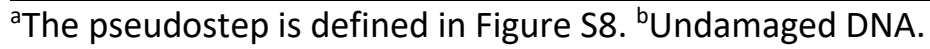


Table S15: Relative deviations in the pseudostep parameters from undamaged DNA, with the number of Xs representing the degree of distortion. ${ }^{\mathrm{a}, \mathrm{b}}$

\begin{tabular}{|c|c|c|c|c|c|c|c|c|}
\hline Adduct & Site(s) & Rise & Shift & Slide & Roll & Tilt & Twist & $\begin{array}{l}\text { Axial } \\
\text { Bend }\end{array}$ \\
\hline \multirow{3}{*}{${ }^{A N} \mathrm{dG}$} & $\mathrm{G}_{1}^{\mathrm{B}}$ & - & - & - & - & - & - & - \\
\hline & $\mathrm{G}_{2}^{\mathrm{B}}$ & - & - & - & - & - & - & - \\
\hline & $\mathrm{G}_{3}^{\mathrm{B}}$ & - & - & - & - & - & - & - \\
\hline \multirow{3}{*}{${ }^{A P} d G$} & $\mathrm{G}_{1}^{\mathrm{B}}$ & - & - & - & - & - & - & - \\
\hline & $\mathrm{G}_{2}^{B}$ & - & - & - & - & - & - & - \\
\hline & $\mathrm{G}_{3}^{\text {B }}$ & - & - & - & - & - & - & - \\
\hline \multirow{3}{*}{${ }^{A N} \mathrm{dG}$} & $\mathrm{G}_{1}^{\mathrm{S}}$ & - & - & $x$ & $x$ & $X X$ & - & - \\
\hline & $\mathrm{G}_{2}^{\mathrm{S}}$ & - & - & - & $X$ & - & - & - \\
\hline & $\mathrm{G}_{3}^{S}$ & - & - & - & $x$ & $x$ & - & - \\
\hline \multirow{3}{*}{${ }^{A P} \mathrm{dG}$} & $\mathrm{G}_{1}^{\mathrm{S}}$ & - & $x$ & - & $x$ & $X$ & $x$ & - \\
\hline & $\mathrm{G}_{2}^{S}$ & - & - & $x$ & $X X$ & - & $x$ & - \\
\hline & $\mathrm{G}_{3}^{\mathrm{s}}$ & - & $x$ & $x$ & $X$ & $x$ & $x$ & - \\
\hline \multirow{3}{*}{${ }^{A N} d G$} & $\mathrm{G}_{1}^{\mathrm{B}} \mathrm{G}_{2}^{\mathrm{B}}$ & - & $x$ & $x$ & - & - & $x$ & - \\
\hline & $\mathrm{G}_{1}^{\mathrm{B}} \mathrm{G}_{3}^{\mathrm{B}}$ & - & - & - & - & - & - & - \\
\hline & $\mathrm{G}_{2}^{\mathrm{B}} \mathrm{G}_{3}^{\mathrm{B}}$ & - & - & $x$ & - & - & - & - \\
\hline \multirow{3}{*}{${ }^{A P} \mathrm{dG}$} & $\mathrm{G}_{1}^{\mathrm{B}} \mathrm{G}_{2}^{\mathrm{B}}$ & - & $x$ & $x$ & - & $x$ & $x$ & - \\
\hline & $\mathrm{G}_{1}^{\mathrm{B}} \mathrm{G}_{3}^{\mathrm{B}}$ & - & - & $x$ & - & - & $x$ & - \\
\hline & $\mathrm{G}_{2}^{\mathrm{B}} \mathrm{G}_{3}^{\mathrm{B}}$ & - & - & $X$ & $X$ & $X$ & $X$ & - \\
\hline \multirow{3}{*}{${ }^{A N} \mathrm{dG}$} & $\mathrm{G}_{1}^{\mathrm{B}} \mathrm{G}_{2}^{\mathrm{S}}$ & - & - & - & - & - & - & - \\
\hline & $\mathrm{G}_{1}^{\mathrm{B}} \mathrm{G}_{3}^{\mathrm{S}}$ & - & - & $X X$ & $x$ & $x$ & $x$ & $x$ \\
\hline & $\mathrm{G}_{2}^{B} \mathrm{G}_{3}^{\mathrm{S}}$ & - & - & $X X$ & $X$ & $X$ & $x$ & - \\
\hline \multirow{3}{*}{${ }^{A P} d G$} & $\mathrm{G}_{1}^{\mathrm{B}} \mathrm{G}_{2}^{\mathrm{S}}$ & - & - & $\mathrm{X}$ & - & - & $X X X$ & - \\
\hline & $\mathrm{G}_{1}^{\mathrm{B}} \mathrm{G}_{3}^{\mathrm{S}}$ & $x$ & - & $X X X$ & $X$ & $x$ & $X X$ & - \\
\hline & $\mathrm{G}_{2}^{\mathrm{B}} \mathrm{G}_{3}^{\mathrm{S}}$ & - & $x$ & $X X$ & $x$ & - & $X X$ & - \\
\hline \multirow{3}{*}{${ }^{A N} d G$} & $\mathrm{G}_{1}^{\mathrm{S}} \mathrm{G}_{2}^{\mathrm{B}}$ & $X$ & $X$ & $X X$ & $X X$ & $X X X$ & $\mathrm{X}$ & $X$ \\
\hline & $\mathrm{G}_{1}^{\mathrm{S}} \mathrm{G}_{3}^{\mathrm{B}}$ & $x$ & $x$ & $X X$ & $X X X$ & $X X$ & - & $x$ \\
\hline & $\mathrm{G}_{2}^{\mathrm{S}} \mathrm{G}_{3}^{\mathrm{B}}$ & - & - & $x$ & $X X X$ & - & - & $X$ \\
\hline \multirow{3}{*}{${ }^{A P} d G$} & $\mathrm{G}_{1}^{\mathrm{S}} \mathrm{G}_{2}^{B}$ & - & $X X$ & $X X$ & $x$ & $x$ & $X X X$ & - \\
\hline & $\mathrm{G}_{1}^{S} \mathrm{G}_{3}^{B}$ & - & - & $x$ & $X X$ & $x$ & $X X$ & - \\
\hline & $\mathrm{G}_{2}^{\mathrm{S}} \mathrm{G}_{3}^{B}$ & - & - & - & $X$ & - & XXXXX & - \\
\hline \multirow{3}{*}{${ }^{A N} d G$} & $\mathrm{G}_{1}^{S} \mathrm{G}_{2}^{\mathrm{S}}$ & - & - & - & - & - & - & - \\
\hline & $\mathrm{G}_{1}^{S} \mathrm{G}_{3}^{S}$ & $X X$ & - & XXXX & $X X X X$ & - & $x$ & $X X$ \\
\hline & $\mathrm{G}_{2}^{\mathrm{S}} \mathrm{G}_{3}^{\mathrm{S}}$ & - & $X$ & $X X X$ & - & - & XXXXX & - \\
\hline \multirow{3}{*}{${ }^{A P} \mathrm{dG}$} & $\mathrm{G}_{1}^{\mathrm{S}} \mathrm{G}_{2}^{\mathrm{S}}$ & - & $X X X$ & $X X X$ & $X X X X$ & $x$ & $X X X X X$ & - \\
\hline & $\mathrm{G}_{1}^{\mathrm{S}} \mathrm{G}_{3}^{\mathrm{S}}$ & $x$ & - & $\mathrm{XXX}$ & $X X$ & - & $\mathrm{XX}$ & $x$ \\
\hline & $\mathrm{G}_{2}^{\mathrm{S}} \mathrm{G}_{3}^{\mathrm{S}}$ & - & $X X$ & XXXXX & XXXXX & $x$ & XXXXX & - \\
\hline
\end{tabular}

${ }^{a}$ The pseudostep is defined in Figure $S 8 .{ }^{b}$ Each $X$ represents a $2 \AA$ or $10^{\circ}$ change from the value in undamaged DNA, with five Xs indicating changes $>12 \AA ̊$ or $60^{\circ}$. 
Table S16: Average (standard deviation in parentheses) solvent accessible surface area of the hydrophobic aryl bulky group (SASA, $\AA^{2}$ ) for monoadducted DNA.

\begin{tabular}{|c|c|c|c|c|c|c|c|}
\hline Adduct & Site & Replica 1 & Replica 2 & Replica 3 & SASA ${ }^{\text {avg, a }}$ & $\begin{array}{c}\text { Dataset } \\
\text { Standard } \\
\text { Deviation }^{\text {b }}\end{array}$ & $\mathrm{BSE}^{\mathrm{C}}$ \\
\hline \multirow{3}{*}{${ }^{A N} d G$} & $\mathrm{G}_{1}^{\mathrm{B}}$ & $117.7(6.1)$ & $119.0(6.9)$ & $118.0(6.5)$ & $118.2(0.7)$ & 6.5 & 0.2 \\
\hline & $\mathrm{G}_{2}^{\mathrm{B}}$ & $117.1(6.0)$ & $117.4(6.1)$ & $116.8(5.9)$ & $117.1(0.3)$ & 6.0 & 0.2 \\
\hline & $\mathrm{G}_{3}^{\mathrm{B}}$ & $118.4(6.0)$ & $118.6(5.9)$ & $118.5(5.8)$ & $118.5(0.1)$ & 5.9 & 0.1 \\
\hline \multirow{3}{*}{${ }^{A P} d G$} & $\mathrm{G}_{1}^{\mathrm{B}}$ & $188.7(6.0)$ & $184.3(7.3)$ & $187.1(6.8)$ & $186.7(2.2)$ & 6.9 & 11.2 \\
\hline & $\mathrm{G}_{2}^{\mathrm{B}}$ & $180.5(6.5)$ & $179.8(6.1)$ & 179.9 (5.7) & $180.0(0.4)$ & 6.1 & 16.5 \\
\hline & $\mathrm{G}_{3}^{\mathrm{B}}$ & $181.0(7.7)$ & $180.6(7.6)$ & $180.3(7.7)$ & $180.6(0.3)$ & 7.6 & 18.8 \\
\hline \multirow{3}{*}{${ }^{A N} d G$} & $\mathrm{G}_{1}^{\mathrm{S}}$ & $66.3(22.1)$ & $59.4(14.2)$ & $86.5(32.6)$ & 70.7 (14.1) & 26.8 & 2.4 \\
\hline & $\mathrm{G}_{2}^{\mathrm{S}}$ & $70.2(23.4)$ & $88.2(25.9)$ & $73.7(25.5)$ & $77.4(9.6)$ & 26.2 & 2.1 \\
\hline & $\mathrm{G}_{3}^{S}$ & $69.2(29.1)$ & $74.9(22.2)$ & $58.7(16.8)$ & $67.6(8.2)$ & 24.2 & 2.6 \\
\hline \multirow{3}{*}{${ }^{A P} d G$} & $\mathrm{G}_{2}^{\mathrm{S}}$ & $106.7(9.9)$ & $109.6(14.8)$ & $113.7(10.9)$ & $110.0(3.5)$ & 12.4 & 0.6 \\
\hline & $\mathrm{G}_{2}^{S}$ & $116.7(21.1)$ & $108.0(11.3)$ & $108.5(11.1)$ & $111.1(4.9)$ & 15.8 & 1.1 \\
\hline & $\mathrm{G}_{3}^{S}$ & $107.0(11.4)$ & $107.0(10.1)$ & $104.3(11.8)$ & $106.1(1.6)$ & 11.2 & 0.7 \\
\hline
\end{tabular}

${ }^{a}$ Average SASA over the three replicates with the standard deviation (parentheses) calculated between the replicas. ${ }^{b}$ Standard deviation calculated over the $1.5 \mu$ s dataset from the three replicas. ${ }^{\text {'Block }}$ standard error (BSE) extrapolated from all frames of the three replicas fitted to a monomolecular growth model. 
Table S17: Average (standard deviation in parentheses) $\chi$ dihedral angle (deg.) for each replica and across all replicas $\left(\chi^{\text {avg }}\right)$ for diadducted DNA. ${ }^{\text {a }}$

\begin{tabular}{|c|c|c|c|c|c|c|c|c|}
\hline Adduct & $\begin{array}{l}\text { Primary } \\
\text { Lesion }\end{array}$ & $\begin{array}{l}\text { Secondary } \\
\text { Lesion }\end{array}$ & Replica 1 & Replica 2 & Replica 3 & $\chi^{\text {avg }}$ & $\mathrm{BSE}^{\mathrm{b}}$ & $\Delta \chi^{\mathrm{c}}$ \\
\hline \multirow{12}{*}{${ }^{A N} \mathrm{dG}$} & \multirow{4}{*}{$\mathrm{G}_{1}^{\mathrm{B}}$} & $\mathrm{GB}$ & $216.4(22.5)$ & $223.1(20.7)$ & $211.2(23.6)$ & $216.9(6.0)$ & 1.6 & -11.9 \\
\hline & & $\mathrm{G}\{$ & $227.1(11.2)$ & $228.6(10.9)$ & $228.0(11.6)$ & $227.9(0.8)$ & 0.3 & -0.9 \\
\hline & & GB & $228.3(15.7)$ & $229.4(14.6)$ & $229.5(14.2)$ & $229.1(0.7)$ & 0.7 & 0.3 \\
\hline & & $\mathrm{G}_{3}$ & $228.3(14.9)$ & $225.1(17.0)$ & $230.9(13.3)$ & $228.1(2.9)$ & 0.7 & -0.7 \\
\hline & \multirow{4}{*}{$\mathrm{GB}$} & $\mathrm{G}_{1}^{B}$ & $226.8(12.3)$ & $227.2(12.1)$ & $226.4(12.7)$ & $226.8(0.4)$ & 0.3 & 2.0 \\
\hline & & GS & $244.1(22.6)$ & $242.5(27.7)$ & $245.1(30.0)$ & $243.9(1.3)$ & 2.3 & 19.1 \\
\hline & & $\mathrm{GB}_{3}^{\mathrm{B}}$ & $220.7(15.8)$ & $217.7(17.8)$ & $218.8(17.2)$ & $219.1(1.5)$ & 1.1 & -5.7 \\
\hline & & $\mathrm{G}_{\hat{S}}$ & $222.6(11.0)$ & $221.3(14.7)$ & $223.2(10.8)$ & $222.4(1.0)$ & 0.6 & -2.4 \\
\hline & \multirow{4}{*}{$\mathrm{GB}$} & $\mathrm{G}_{1}^{\mathrm{B}}$ & $231.0(12.6)$ & $231.5(12.3)$ & $230.6(12.6)$ & $231.0(0.5)$ & 0.2 & 0.0 \\
\hline & & GS & $228.9(14.4)$ & $229.5(13.1)$ & $229.1(13.3)$ & $229.2(0.3)$ & 0.4 & -1.8 \\
\hline & & $\mathrm{GB}$ & $224.4(16.7)$ & $227.2(14.4)$ & $227.0(14.5)$ & $226.2(1.6)$ & 0.8 & -4.8 \\
\hline & & $\mathrm{G}\{$ & $229.9(14.8)$ & $227.4(15.8)$ & $228.6(15.5)$ & $228.6(1.3)$ & 0.7 & -2.4 \\
\hline \multirow{12}{*}{${ }^{A P} \mathrm{dG}$} & \multirow{4}{*}{$\mathrm{G}_{1}^{B}$} & $\mathrm{~GB}$ & $191.3(21.7)$ & $189.8(20.6)$ & $183.4(15.8)$ & $188.2(4.2)$ & 12.6 & -14.5 \\
\hline & & $\mathrm{G}\{$ & $222.8(19.8)$ & $222.2(21.6)$ & $211.1(24.9)$ & $218.7(6.6)$ & 1.8 & 16.0 \\
\hline & & $\mathrm{GB}$ & $201.0(20.3)$ & $198.6(19.0)$ & $200.3(19.6)$ & $200.0(1.2)$ & 2.0 & -2.8 \\
\hline & & $\mathrm{G}\}$ & $211.7(20.0)$ & $209.7(20.6)$ & $210.6(20.5)$ & $210.7(1.0)$ & 2.0 & 8.0 \\
\hline & \multirow{4}{*}{$\mathrm{G}_{2}^{B}$} & $\mathrm{G}_{1}^{\mathrm{B}}$ & $214.4(18.3)$ & $216.8(14.4)$ & $217.4(13.2)$ & 216.2 (1.6) & 3.4 & -1.9 \\
\hline & & $\mathrm{G}_{1}^{\mathfrak{s}}$ & $221.5(18.1)$ & $218.2(20.3)$ & $216.3(19.5)$ & $218.7(2.6)$ & 2.4 & 0.5 \\
\hline & & $\mathrm{GB}_{3}^{\mathrm{B}}$ & $201.4(20.3)$ & $215.1(14.8)$ & $212.4(16.7)$ & $209.6(7.3)$ & 1.4 & -8.5 \\
\hline & & $\mathrm{G}_{3}$ & $216.3(15.0)$ & $210.2(19.1)$ & $216.3(15.5)$ & $214.3(3.5)$ & 1.2 & -3.9 \\
\hline & \multirow{4}{*}{$\mathrm{G}_{3}^{B}$} & $\mathrm{G}_{1}^{\mathrm{B}}$ & $220.8(20.1)$ & $209.4(22.7)$ & $212.1(22.0)$ & $214.1(6.0)$ & 3.2 & -3.6 \\
\hline & & $\mathrm{G}_{1}$ & $218.4(18.4)$ & $210.5(22.1)$ & $203.8(21.1)$ & $210.9(7.3)$ & 3.2 & -6.8 \\
\hline & & $\mathrm{GB}$ & $204.5(25.4)$ & $203.9(24.0)$ & $196.8(22.4)$ & $201.8(4.3)$ & 3.8 & -15.9 \\
\hline & & $\mathrm{G}\{$ & 203.5 (19.6) & $211.3(20.8)$ & $207.2(21.0)$ & $207.3(3.9)$ & 1.9 & -10.4 \\
\hline \multirow{12}{*}{${ }^{A N} \mathrm{dG}$} & \multirow{4}{*}{$\mathrm{G}_{1}$} & $\mathrm{~GB}$ & $54.5(14.3)$ & $56.9(13.4)$ & $57.3(13.6)$ & $56.2(1.5)$ & 0.5 & -1.4 \\
\hline & & $\mathrm{G}\{$ & $53.4(16.8)$ & $48.5(14.2)$ & $49.9(15.5)$ & $50.6(2.5)$ & 0.3 & -7.1 \\
\hline & & $\mathrm{GB}_{3}$ & $49.4(14.6)$ & $48.8(13.1)$ & $47.3(14.1)$ & $48.5(1.1)$ & 0.6 & -9.1 \\
\hline & & $\mathrm{G}_{\}}$ & $50.1(14.7)$ & $48.0(13.9)$ & $55.3(18.2)$ & $51.1(3.7)$ & 0.3 & -6.5 \\
\hline & \multirow{4}{*}{$\mathrm{G}\{$} & $\mathrm{G}_{1}^{\mathrm{B}}$ & $65.0(16.8)$ & $68.0(15.7)$ & $67.7(15.1)$ & $66.9(1.6)$ & 0.4 & 14.4 \\
\hline & & $\mathrm{GS}_{1}$ & $57.6(12.8)$ & $61.9(12.8)$ & $62.0(12.6)$ & $60.5(2.5)$ & 0.5 & 8.0 \\
\hline & & $\mathrm{GB}_{3}^{\mathrm{B}}$ & $43.3(14.0)$ & $53.6(17.6)$ & $43.6(14.0)$ & $46.8(5.8)$ & 0.8 & -5.7 \\
\hline & & $\mathrm{G}_{3}$ & $53.5(23.7)$ & $70.0(36.1)$ & $101.0(34.4)$ & $74.8(24.1)$ & 3.3 & 22.3 \\
\hline & \multirow{4}{*}{$\mathrm{G}\}$} & $\mathrm{G}_{1}^{\mathrm{B}}$ & $49.0(17.2)$ & $44.0(14.8)$ & $47.7(14.3)$ & $46.9(2.6)$ & 0.6 & -2.9 \\
\hline & & GS & $44.6(15.3)$ & 49.9 (15.1) & $46.8(16.0)$ & $47.1(2.6)$ & 0.6 & -2.7 \\
\hline & & $\mathrm{GB}$ & 44.5 (15.1) & $60.1(17.3)$ & $44.7(15.2)$ & $49.8(9.0)$ & 1.0 & -0.1 \\
\hline & & $\mathrm{G}\{$ & $52.4(15.9)$ & $64.7(15.7)$ & $69.9(14.1)$ & $62.3(8.9)$ & 1.0 & 12.5 \\
\hline \multirow{12}{*}{${ }^{A P} \mathrm{dG}$} & \multirow{4}{*}{$\mathrm{G}_{1}^{\mathfrak{S}}$} & $\mathrm{GB}$ & $41.1(13.3)$ & $44.4(24.6)$ & $53.3(35.9)$ & $46.2(6.3)$ & 2.2 & 2.6 \\
\hline & & $\mathrm{G}\{$ & $146.9(26.0)$ & 129.5 (37.9) & $139.5(21.5)$ & $138.6(8.8)$ & 5.1 & 95.0 \\
\hline & & $\mathrm{G}_{3}^{\mathrm{B}}$ & $45.8(19.9)$ & $42.1(19.2)$ & 40.6 (17.9) & $42.9(2.7)$ & 0.5 & -0.8 \\
\hline & & $\mathrm{G}_{3}$ & $45.1(24.3)$ & $50.5(41.2)$ & $43.6(18.9)$ & $46.4(3.6)$ & 1.7 & 2.8 \\
\hline & \multirow{4}{*}{$\mathrm{G}\{$} & $\mathrm{G}_{1}^{\mathrm{B}}$ & $174.1(20.6)$ & $176.3(18.6)$ & $170.1(24.9)$ & $173.5(3.1)$ & 10.3 & 123.9 \\
\hline & & GS & $190.8(16.0)$ & 183.7 (17.9) & $188.7(16.4)$ & $187.7(3.7)$ & 14.8 & 138.2 \\
\hline & & GB & $64.7(35.1)$ & $43.0(25.7)$ & $46.2(32.0)$ & $51.3(11.7)$ & 2.6 & 1.7 \\
\hline & & $\mathrm{G}_{\xi}$ & $131.4(32.4)$ & $135.5(15.6)$ & $132.2(17.3)$ & $133.1(2.2)$ & 5.2 & 83.5 \\
\hline & \multirow{4}{*}{$\mathrm{G} \xi$} & $\mathrm{GB}_{1}^{\mathrm{B}}$ & $37.8(16.4)$ & $37.4(15.5)$ & $37.0(15.5)$ & $37.4(0.4)$ & 0.3 & -4.3 \\
\hline & & $\mathrm{GS}_{1}$ & $43.6(27.2)$ & $45.2(41.5)$ & 41.5 (21.9) & $43.4(1.9)$ & 2.7 & 1.8 \\
\hline & & $\mathrm{GB}$ & $39.1(29.5)$ & $36.1(18.0)$ & $47.4(45.1)$ & 40.9 (5.9) & 2.8 & -0.8 \\
\hline & & $\mathrm{G}\{$ & $170.3(18.9)$ & $171.7(18.7)$ & $177.7(21.0)$ & $173.2(4.0)$ & 15.6 & 131.6 \\
\hline
\end{tabular}

${ }^{a} \chi=\angle\left(O 4^{\prime}-C 1^{\prime}-N 9-C 4\right)$. See Figure 1 in the main text. ${ }^{b}$ Block standard error (BSE) extrapolated from all frames of the three replicas fitted to a monomolecular growth model. 'Calculated as the difference in $\chi^{\text {avg }}$ for the lesion in diadducted DNA minus monoadducted DNA. 
Table S18: Average (standard deviation in parentheses) $\theta$ dihedral angle (deg.) for each replica and across all replicas $\left(\theta^{\text {avg }}\right)$ for diadducted DNA. ${ }^{a}$

\begin{tabular}{|c|c|c|c|c|c|c|c|c|}
\hline Adduct & $\begin{array}{l}\text { Primary } \\
\text { Lesion }\end{array}$ & $\begin{array}{l}\text { Secondary } \\
\text { Lesion }\end{array}$ & Replica 1 & Replica 2 & Replica 3 & $\theta^{\text {avg }}$ & $\mathrm{BSE}^{\mathrm{b}}$ & $\Delta \theta^{c}$ \\
\hline \multirow{12}{*}{${ }^{\mathrm{AN}} \mathrm{dG}$} & \multirow{4}{*}{$\mathrm{G}_{1}^{B}$} & $\mathrm{~GB}$ & $141.5(37.3)$ & $134.9(35.0)$ & $149.9(39.6)$ & $142.1(7.5)$ & 7.2 & 14.0 \\
\hline & & $\mathrm{G}\{$ & $144.8(21.3)$ & $139.8(19.3)$ & $139.9(20.2)$ & $141.5(2.9)$ & 1.2 & 13.4 \\
\hline & & $\mathrm{G}_{3}^{\mathrm{B}}$ & $127.4(27.9)$ & $125.2(25.8)$ & $125.5(25.0)$ & $126.0(1.2)$ & 1.5 & -2.1 \\
\hline & & $\mathrm{G}_{3}$ & $130.4(26.7)$ & $135.5(29.7)$ & $127.6(23.9)$ & $131.2(4.0)$ & 1.0 & 3.0 \\
\hline & \multirow{4}{*}{$\mathrm{GB}$} & $\mathrm{G}_{1}^{B}$ & $143.8(24.1)$ & $141.2(23.0)$ & $146.6(25.2)$ & $143.9(2.7)$ & 0.9 & 1.6 \\
\hline & & G\{ & $134.7(21.7)$ & $136.4(18.3)$ & $138.7(24.1)$ & $136.6(2.0)$ & 1.5 & -5.7 \\
\hline & & $\mathrm{GB}_{3}^{\mathrm{B}}$ & $147.8(27.7)$ & $150.0(29.6)$ & $149.3(29.2)$ & $149.0(1.1)$ & 6.0 & 6.7 \\
\hline & & $\mathrm{G}_{3}$ & $148.9(22.0)$ & $148.3(26.8)$ & $147.7(22.2)$ & $148.3(0.6)$ & 3.6 & 6.0 \\
\hline & \multirow{4}{*}{$\mathrm{GB}$} & $\mathrm{G}_{1}^{\mathrm{B}}$ & $132.8(19.2)$ & $132.8(18.6)$ & $133.1(19.3)$ & $132.9(0.2)$ & 0.2 & 0.3 \\
\hline & & GS & $135.6(21.7)$ & $134.4(20.6)$ & $134.6(20.8)$ & $134.9(0.7)$ & 0.5 & 2.3 \\
\hline & & $\mathrm{GB}$ & $139.2(26.4)$ & $135.4(22.9)$ & $135.4(23.8)$ & $136.7(2.2)$ & 1.1 & 4.1 \\
\hline & & $\mathrm{G}\{$ & $130.0(22.3)$ & $133.2(27.0)$ & $131.6(23.4)$ & $131.6(1.6)$ & 2.5 & -1.0 \\
\hline \multirow{12}{*}{ APdG } & \multirow{4}{*}{$\mathrm{G}_{1}^{B}$} & $\mathrm{G}_{2}^{B}$ & $185.3(19.9)$ & $186.0(18.7)$ & $190.1(16.5)$ & $187.1(2.6)$ & 13.6 & 10.0 \\
\hline & & $\mathrm{G}\{$ & $174.2(18.6)$ & $172.7(18.7)$ & $180.0(20.4)$ & $175.7(3.9)$ & 16.5 & -1.5 \\
\hline & & $\mathrm{G}_{3}^{B}$ & $178.3(41.3)$ & $180.8(40.2)$ & $174.7(43.0)$ & $178.0(3.1)$ & 18.4 & 0.8 \\
\hline & & $\mathrm{G}\}$ & $167.6(39.8)$ & $172.5(38.2)$ & $170.0(38.4)$ & $170.1(2.4)$ & 15.9 & -7.1 \\
\hline & \multirow{4}{*}{$\mathrm{G} B$} & $\mathrm{G}_{1}^{B}$ & $176.0(16.9)$ & $175.3(16.1)$ & $177.0(15.3)$ & $176.1(0.9)$ & 14.7 & 4.3 \\
\hline & & $\mathrm{G}_{1}$ & $169.6(21.6)$ & $171.3(21.9)$ & $173.5(25.0)$ & $171.5(2.0)$ & 13.9 & -0.3 \\
\hline & & $\mathrm{G}_{3}^{\mathrm{B}}$ & $175.6(29.1)$ & $168.6(24.4)$ & $170.3(23.6)$ & $171.5(3.7)$ & 16.9 & -0.3 \\
\hline & & $\mathrm{G}_{\hat{S}}$ & $174.2(20.9)$ & $177.1(23.0)$ & $174.6(20.9)$ & $175.3(1.6)$ & 15.3 & 3.5 \\
\hline & \multirow{4}{*}{$\mathrm{GB}$} & $\mathrm{G}_{1}^{\mathrm{B}}$ & $156.4(32.4)$ & $167.2(36.5)$ & $163.6(36.0)$ & $162.4(5.5)$ & 14.6 & 5.2 \\
\hline & & GS & $163.5(31.1)$ & $169.0(34.9)$ & $176.7(33.5)$ & $169.7(6.6)$ & 16.7 & 12.5 \\
\hline & & $\mathrm{GB}$ & $172.3(35.4)$ & $176.2(31.1)$ & $183.2(29.7)$ & $177.3(5.5)$ & 17.9 & 20.1 \\
\hline & & $\mathrm{G}\{$ & $155.4(38.7)$ & $165.1(37.6)$ & $168.1(39.3)$ & $162.9(6.7)$ & 13.4 & 5.7 \\
\hline \multirow{12}{*}{${ }^{A N} \mathrm{dG}$} & \multirow{4}{*}{$\mathrm{G}_{1}^{\mathfrak{S}}$} & $\mathrm{GB}$ & $117.3(20.7)$ & $99.6(21.5)$ & $99.8(46.5)$ & $105.6(10.2)$ & 6.7 & -141.3 \\
\hline & & $\mathrm{G}\{$ & $246.5(81.7)$ & $253.9(29.0)$ & $250.2(60.0)$ & $250.2(3.7)$ & 4.6 & 3.3 \\
\hline & & $\mathrm{GB}_{3}$ & $251.0(58.1)$ & $254.8(30.1)$ & $248.4(43.8)$ & $251.4(3.2)$ & 8.0 & 4.6 \\
\hline & & $\mathrm{G}_{3}$ & $249.7(60.9)$ & $249.4(34.4)$ & $101.0(51.1)$ & $200.0(85.8)$ & 8.7 & -46.8 \\
\hline & \multirow{4}{*}{$\mathrm{G}\{$} & $\mathrm{G}_{1}^{B}$ & $157.0(83.6)$ & $89.8(68.2)$ & 93.9 (72.9) & $113.6(37.7)$ & 10.7 & -82.9 \\
\hline & & $\mathrm{G}_{1}$ & $97.9(25.3)$ & $91.7(18.8)$ & $91.8(20.5)$ & $93.8(3.6)$ & 1.1 & -102.7 \\
\hline & & $\mathrm{GB}_{3}^{\mathrm{B}}$ & $261.5(22.3)$ & $126.7(88.3)$ & $262.3(22.2)$ & $216.8(78.0)$ & 8.2 & 20.4 \\
\hline & & $\mathrm{G}_{3}$ & $261.7(39.9)$ & $254.0(41.9)$ & $222.0(38.9)$ & $245.9(21.1)$ & 5.0 & 49.4 \\
\hline & \multirow{4}{*}{$\mathrm{G}_{3}$} & $\mathrm{G}_{1}^{\mathrm{B}}$ & $245.0(74.8)$ & $246.2(28.2)$ & 265.7 (39.1) & $252.3(11.6)$ & 7.5 & 56.2 \\
\hline & & $\mathrm{G}_{1}$ & $248.2(29.7)$ & $267.4(67.1)$ & $246.4(58.9)$ & $254.0(11.6)$ & 8.0 & 57.9 \\
\hline & & $\mathrm{GB}$ & $246.4(22.5)$ & $89.6(72.3)$ & $241.8(40.2)$ & $192.6(89.2)$ & 10.3 & -3.5 \\
\hline & & $\mathrm{G}\{$ & $262.9(52.0)$ & $91.6(57.9)$ & $98.1(80.9)$ & $150.8(97.1)$ & 11.1 & -45.3 \\
\hline \multirow{12}{*}{${ }^{A P} \mathrm{dG}$} & \multirow{4}{*}{$\mathrm{G}_{1}^{\mathfrak{S}}$} & $\mathrm{GB}$ & $184.9(18.8)$ & $185.6(19.5)$ & $188.1(20.1)$ & $186.2(1.7)$ & 16.1 & -0.1 \\
\hline & & $\mathrm{G}_{2}$ & $179.1(24.2)$ & $187.4(19.0)$ & $188.8(18.7)$ & $185.1(5.2)$ & 14.4 & -1.2 \\
\hline & & $\mathrm{GB}_{3}$ & $206.2(38.2)$ & $178.3(28.7)$ & $172.8(23.2)$ & $185.8(17.9)$ & 18.2 & -0.5 \\
\hline & & $\mathrm{GS}_{3}$ & $213.4(36.2)$ & $179.6(26.2)$ & 189.9 (35.9) & $194.3(17.3)$ & 17.3 & 8.0 \\
\hline & \multirow{4}{*}{$\mathrm{G}\{$} & $\mathrm{G}_{1}^{\mathrm{B}}$ & $174.0(14.4)$ & $174.8(15.2)$ & $172.9(16.4)$ & $173.9(0.9)$ & 1.5 & -20.1 \\
\hline & & $\mathrm{G}\{$ & $168.3(15.8)$ & 172.5 (13.9) & $171.2(14.5)$ & $170.7(2.1)$ & 12.2 & -23.3 \\
\hline & & $\mathrm{GB}_{3}^{B}$ & $194.9(24.1)$ & $189.0(32.5)$ & $187.0(29.5)$ & $190.3(4.1)$ & 17.0 & -3.7 \\
\hline & & $\mathrm{G}_{3}$ & $195.2(16.9)$ & $202.7(14.4)$ & $198.0(14.0)$ & $198.6(3.7)$ & 5.7 & 4.6 \\
\hline & \multirow{4}{*}{$\mathrm{G}$} & $\mathrm{G}_{1}^{B}$ & $195.7(28.6)$ & $205.3(27.2)$ & $202.4(26.2)$ & $201.1(5.0)$ & 11.8 & 13.2 \\
\hline & & G\{ & $220.3(28.9)$ & $206.6(28.8)$ & $219.2(27.8)$ & $215.4(7.6)$ & 6.5 & 27.5 \\
\hline & & $\mathrm{GB}$ & $204.2(25.1)$ & $197.5(27.9)$ & $198.0(27.6)$ & 199.9 (3.7) & 12.2 & 12.0 \\
\hline & & $\mathrm{G}\{$ & $167.6(19.3)$ & $180.5(23.3)$ & $166.9(18.2)$ & $171.7(7.7)$ & 13.9 & -16.2 \\
\hline
\end{tabular}

${ }^{\mathrm{a}} \theta=\angle$ (N9-C8-N10-C10). See Figure 1 in the main text. ${ }^{\mathrm{b}}$ Block standard error (BSE) extrapolated from all frames of the three replicas fitted to a monomolecular growth model. 'Calculated as the difference in $\theta^{\text {avg }}$ for the lesion in diadducted DNA minus monoadducted DNA. 
Table S19: Average (standard deviation in parentheses) $\phi$ dihedral angle (deg.) for each replica and across all replicas $\left(\phi^{\text {avg }}\right)$ for diadducted DNA. ${ }^{a}$

\begin{tabular}{|c|c|c|c|c|c|c|c|c|}
\hline Adduct & $\begin{array}{l}\text { Primary } \\
\text { Lesion }\end{array}$ & $\begin{array}{l}\text { Secondary } \\
\text { Lesion }\end{array}$ & Replica 1 & Replica 2 & Replica 3 & $\phi^{\text {avg }}$ & $\mathrm{BSE}^{\mathrm{b}}$ & $\Delta \phi^{\mathrm{c}}$ \\
\hline \multirow{12}{*}{${ }^{A N} \mathrm{dG}$} & \multirow{4}{*}{$\mathrm{G}_{1}^{\mathrm{B}}$} & $\mathrm{GB}$ & 359.5 (12.8) & $359.9(13.1)$ & $359.7(12.3)$ & $359.7(0.2)$ & 0.4 & -0.8 \\
\hline & & $\mathrm{G}\{$ & $4.9(12.3)$ & $3.1(11.8)$ & $2.6(11.9)$ & $3.5(1.2)$ & 0.3 & 3.0 \\
\hline & & GB & $0.6(11.7)$ & $0.4(11.9)$ & 359.9 (11.9) & $0.3(0.3)$ & 0.8 & -0.2 \\
\hline & & $\mathrm{G}_{3}$ & $1.1(12.0)$ & $1.0(12.2)$ & $0.8(12.0)$ & $1.0(0.1)$ & 0.1 & 0.5 \\
\hline & \multirow{4}{*}{$\mathrm{GB}$} & $\mathrm{G}_{1}^{B}$ & $4.8(11.6)$ & 4.9 (11.9) & $5.2(11.6)$ & $5.0(0.2)$ & 0.2 & -1.1 \\
\hline & & GS & 358.7 (11.7) & $0.8(11.5)$ & $1.4(11.7)$ & $0.3(1.2)$ & 0.2 & -5.8 \\
\hline & & $\mathrm{GB}$ & $6.0(12.4)$ & $5.3(12.4)$ & $5.5(12.4)$ & $5.6(0.4)$ & 0.2 & -0.5 \\
\hline & & $\mathrm{G}_{3}$ & $8.0(12.2)$ & $6.8(12.5)$ & 7.9 (12.3) & $7.5(0.6)$ & 0.1 & 1.5 \\
\hline & \multirow{4}{*}{$\mathrm{GB}$} & $\mathrm{G}_{1}^{\mathrm{B}}$ & $2.7(12.0)$ & $2.3(12.0)$ & $2.6(12.1)$ & $2.5(0.2)$ & 0.2 & -0.1 \\
\hline & & GS & $2.6(12.2)$ & $3.3(12.1)$ & $2.8(12.2)$ & $2.9(0.3)$ & 0.1 & 0.3 \\
\hline & & $\mathrm{GB}$ & $2.6(12.2)$ & $3.0(12.0)$ & $2.8(12.1)$ & $2.8(0.2)$ & 0.2 & 0.2 \\
\hline & & $\mathrm{G}\{$ & $1.0(12.0)$ & $1.8(12.2)$ & $0.4(12.2)$ & $1.1(0.7)$ & 1.6 & -1.6 \\
\hline \multirow{12}{*}{${ }^{A P} \mathrm{dG}$} & \multirow{4}{*}{$\mathrm{GB}_{1}^{\mathrm{B}}$} & $\mathrm{GB}$ & $10.5(11.6)$ & $10.3(11.4)$ & 8.4 (10.9) & $9.7(1.1)$ & 0.4 & 4.2 \\
\hline & & $\mathrm{G}\{$ & $12.0(11.6)$ & $13.1(11.4)$ & $11.0(11.6)$ & $12.0(1.1)$ & 0.3 & 6.5 \\
\hline & & $\mathrm{G}_{3}^{\mathrm{B}}$ & $4.5(12.4)$ & $5.4(12.6)$ & $4.3(12.7)$ & $4.8(0.6)$ & 0.3 & -0.7 \\
\hline & & $\mathrm{G}\}$ & $7.4(13.8)$ & $7.9(13.4)$ & $8.2(13.5)$ & $7.8(0.4)$ & 0.6 & 2.3 \\
\hline & \multirow{4}{*}{$\mathrm{G}_{2}^{\mathrm{B}}$} & $\mathrm{GB}_{1}^{\mathrm{B}}$ & $11.5(11.7)$ & $11.5(11.6)$ & $10.2(11.3)$ & $11.1(0.7)$ & 0.5 & -6.2 \\
\hline & & $\mathrm{G}_{1}^{\mathrm{S}}$ & $8.6(12.3)$ & $8.3(12.6)$ & $7.3(12.4)$ & $8.0(0.7)$ & 0.4 & -9.3 \\
\hline & & $\mathrm{GB}$ & $13.9(12.9)$ & $17.3(12.3)$ & $16.6(12.1)$ & $15.9(1.8)$ & 0.5 & -1.4 \\
\hline & & $\mathrm{G}_{\xi}$ & $16.7(12.1)$ & $15.3(12.5)$ & $16.8(12.0)$ & $16.3(0.8)$ & 0.4 & -1.1 \\
\hline & \multirow{4}{*}{$\mathrm{GB}_{3}^{\mathrm{B}}$} & $\mathrm{G}_{1}^{\mathrm{B}}$ & $11.8(12.9)$ & $8.7(13.1)$ & $8.6(13.1)$ & $9.7(1.8)$ & 0.5 & -1.2 \\
\hline & & $\mathrm{GS}_{1}$ & $12.2(13.3)$ & $9.6(13.2)$ & $9.2(13.2)$ & $10.3(1.6)$ & 0.5 & -0.6 \\
\hline & & $\mathrm{GB}$ & $8.3(12.3)$ & $10.6(12.6)$ & $7.8(12.3)$ & $8.9(1.5)$ & 0.4 & -2.0 \\
\hline & & $\mathrm{G}\{$ & $358.9(13.2)$ & $5.6(13.6)$ & $3.9(13.7)$ & $2.8(2.8)$ & 0.6 & -8.1 \\
\hline \multirow{12}{*}{${ }^{\mathrm{AN}} \mathrm{dG}$} & \multirow{4}{*}{$\mathrm{G}_{1}^{S}$} & $\mathrm{~GB}$ & $358.0(10.7)$ & $359.6(10.1)$ & $359.6(10.1)$ & $359.1(0.9)$ & 0.3 & -3.9 \\
\hline & & $\mathrm{G}\{$ & $2.1(10.5)$ & $1.6(10.6)$ & $1.5(10.6)$ & $1.7(0.3)$ & 0.2 & -1.2 \\
\hline & & $\mathrm{G}_{3}^{B}$ & $1.5(10.7)$ & $2.2(10.5)$ & $1.4(10.6)$ & $1.7(0.4)$ & 0.1 & -1.2 \\
\hline & & $\mathrm{GS}_{3}$ & $1.8(10.5)$ & $1.8(10.7)$ & $0.1(10.6)$ & $1.2(1.0)$ & 0.8 & -1.7 \\
\hline & \multirow{4}{*}{$\mathrm{G}\{$} & $\mathrm{GB}_{1}^{\mathrm{B}}$ & $0.9(10.8)$ & $1.2(10.8)$ & $1.1(10.7)$ & $1.0(0.2)$ & 0.2 & 1.1 \\
\hline & & GS & $359.9(10.7)$ & $1.7(10.7)$ & $1.6(10.7)$ & $1.1(0.8)$ & 0.2 & 1.1 \\
\hline & & $\mathrm{GB}_{3}^{\mathrm{B}}$ & $358.6(10.2)$ & $359.8(10.8)$ & $358.5(10.1)$ & $359.0(0.8)$ & 0.2 & -1.0 \\
\hline & & $\mathrm{G}_{3}$ & $2.4(11.3)$ & $2.4(11.0)$ & $1.5(10.9)$ & $2.1(0.5)$ & 0.2 & 2.2 \\
\hline & \multirow{4}{*}{$\mathrm{G}_{\mathfrak{s}}$} & $\mathrm{G}_{1}^{\mathrm{B}}$ & $180.9(9.6)$ & $180.9(9.5)$ & $184.9(10.4)$ & $182.2(2.3)$ & 13.0 & 1.2 \\
\hline & & G\{ & $180.5(9.5)$ & $183.4(10.6)$ & $180.7(9.5)$ & 181.5 (1.6) & 14.8 & 0.5 \\
\hline & & $\mathrm{GB}$ & $181.6(9.3)$ & $180.5(9.9)$ & $181.0(9.4)$ & $181.0(0.6)$ & 14.5 & 0.1 \\
\hline & & $\mathrm{G}\{$ & $183.9(10.8)$ & $181.2(10.5)$ & $180.9(10.6)$ & $182.0(1.6)$ & 15.7 & 1.0 \\
\hline \multirow{12}{*}{${ }^{A P} \mathrm{dG}$} & \multirow{4}{*}{$\mathrm{G}_{1}$} & $\mathrm{~GB}$ & $353.3(11.0)$ & $353.9(11.1)$ & $354.5(11.2)$ & $353.9(0.6)$ & 0.2 & -2.4 \\
\hline & & $\mathrm{G}\{$ & $1.7(11.7)$ & $2.3(11.5)$ & $2.9(11.3)$ & $2.3(0.6)$ & 0.3 & 6.0 \\
\hline & & $\mathrm{GB}_{3}^{\mathrm{B}}$ & $355.9(11.0)$ & $356.3(11.2)$ & $356.1(11.2)$ & $356.1(0.2)$ & 0.1 & -0.2 \\
\hline & & $\mathrm{G}_{3}$ & $357.3(11.0)$ & $356.0(11.8)$ & $356.6(11.2)$ & $356.6(0.6)$ & 0.2 & 0.3 \\
\hline & \multirow{4}{*}{$\mathrm{G}\{$} & $\mathrm{G}_{1}^{\mathrm{B}}$ & $354.3(11.8)$ & $353.2(11.6)$ & $355.8(12.3)$ & $354.4(1.3)$ & 0.4 & 0.7 \\
\hline & & GS & $358.1(11.1)$ & $358.6(11.4)$ & $358.9(11.2)$ & $358.5(0.4)$ & 0.4 & 4.8 \\
\hline & & $\mathrm{G}_{3}^{B}$ & $355.1(12.4)$ & $353.6(11.6)$ & $354.2(12.1)$ & $354.3(0.7)$ & 0.5 & 0.6 \\
\hline & & $\mathrm{G}_{3}$ & $9.0(12.7)$ & $3.2(12.1)$ & 9.7 (11.9) & $7.3(3.5)$ & 0.7 & 13.6 \\
\hline & \multirow{4}{*}{$\mathrm{G}\}$} & $\mathrm{G}_{1}^{B}$ & $352.6(11.1)$ & $352.7(11.2)$ & $352.5(11.1)$ & $352.6(0.1)$ & 0.1 & -0.3 \\
\hline & & G\{ & 355.5 (11.7) & $354.4(11.7)$ & $354.7(11.2)$ & $354.9(0.6)$ & 0.4 & 2.0 \\
\hline & & $\mathrm{GB}$ & 354.5 (11.1) & $354.1(11.0)$ & 356.2 (11.9) & $354.9(1.1)$ & 0.7 & 2.1 \\
\hline & & $\mathrm{G}\{$ & $2.1(13.2)$ & $2.4(14.1)$ & $358.6(13.4)$ & $1.1(1.7)$ & 0.6 & 8.2 \\
\hline
\end{tabular}

${ }^{\mathrm{a}} \phi=\angle(\mathrm{C} 8-\mathrm{N} 10-\mathrm{C} 10-\mathrm{C} 11)$. See Figure 1 in the main text. ${ }^{\mathrm{b}}$ Block standard error (BSE) extrapolated from all frames of the three replicas fitted to a monomolecular growth model. ${ }^{~ C}$ Calculated as the difference in $\phi^{\text {avg }}$ for the lesion in diadducted DNA minus monoadducted DNA. 
Table S20: Average (standard deviation in parentheses) solvent accessible surface area of the hydrophobic aryl bulky group (SASA, $\AA^{2}$ ) for diadducted DNA.

\begin{tabular}{|c|c|c|c|c|c|c|c|}
\hline \multirow[b]{2}{*}{$\begin{array}{l}\text { Primary } \\
\text { Lesion }\end{array}$} & \multirow[b]{2}{*}{$\begin{array}{c}\text { Secondary } \\
\text { Lesion }^{\mathrm{a}}\end{array}$} & \multicolumn{3}{|c|}{${ }^{A N} \mathrm{dG}$} & \multicolumn{3}{|c|}{ APdG } \\
\hline & & SASA ${ }^{\text {avg b }}$ & $\begin{array}{c}\text { Dataset } \\
\text { Standard } \\
\text { Deviation }^{c}\end{array}$ & $\mathrm{BSE}^{\mathrm{d}}$ & SASA ${ }^{\text {avg b }}$ & $\begin{array}{c}\text { Dataset } \\
\text { Standard } \\
\text { Deviation }^{c}\end{array}$ & $\mathrm{BSE}^{\mathrm{d}}$ \\
\hline \multirow{5}{*}{$\mathrm{G}_{1}^{B}$} & absent & $118.2(0.7)$ & 6.5 & 0.2 & $186.7(2.2)$ & 6.9 & 11.2 \\
\hline & $\mathrm{G}_{2}^{\mathrm{B}}$ & $103.1(2.9)$ & 11.9 & 0.7 & $164.0(0.8)$ & 6.1 & 0.3 \\
\hline & $\mathrm{G}_{2}^{\mathrm{S}}$ & $115.4(1.7)$ & 6.7 & 0.3 & $144.4(1.7)$ & 12.2 & 0.7 \\
\hline & $\mathrm{G}_{3}^{B}$ & $118.1(0.1)$ & 6.4 & 0.7 & $187.3(0.2)$ & 6.8 & 7.7 \\
\hline & $\mathrm{G}_{3}^{S}$ & $118.4(0.7)$ & 6.8 & 0.8 & $184.0(0.0)$ & 7.8 & 18.7 \\
\hline \multirow{5}{*}{$\mathrm{G}_{2}^{B}$} & absent & $117.1(0.3)$ & 6.0 & 0.2 & $180.0(0.4)$ & 6.1 & 16.5 \\
\hline & $\mathrm{G}_{1}^{\mathrm{B}}$ & $95.0(0.3)$ & 10.7 & 0.2 & $149.0(0.6)$ & 7.5 & 0.4 \\
\hline & $\mathrm{G}_{1}^{\mathrm{S}}$ & 104.9 (1.9) & 12.0 & 0.8 & $161.8(0.7)$ & 8.0 & 0.5 \\
\hline & $\mathrm{G}_{3}^{\mathrm{B}}$ & $118.6(0.6)$ & 6.6 & 0.3 & $172.4(2.4)$ & 15.9 & 16.8 \\
\hline & $\mathrm{G}_{3}^{S}$ & $115.6(1.3)$ & 6.4 & 0.2 & $179.5(1.4)$ & 7.9 & 16.2 \\
\hline \multirow{5}{*}{$\mathrm{G}_{3}^{\mathrm{B}}$} & absent & $118.5(0.1)$ & 5.9 & 0.1 & $180.6(0.3)$ & 7.6 & 18.8 \\
\hline & $\mathrm{G}_{1}^{\mathrm{B}}$ & $118.4(0.1)$ & 6.0 & 0.1 & $181.8(1.7)$ & 7.9 & 19.2 \\
\hline & $\mathrm{G}_{1}^{\mathrm{S}}$ & $117.7(0.2)$ & 6.5 & 0.1 & 182.1 (1.9) & 8.0 & 19.0 \\
\hline & $\mathrm{G}_{2}^{B}$ & $118.3(0.2)$ & 6.7 & 0.2 & $168.1(0.6)$ & 18.4 & 5.7 \\
\hline & $\mathrm{G}_{2}^{S}$ & $118.9(0.5)$ & 7.2 & 1.6 & $176.1(10.2)$ & 18.2 & 18.0 \\
\hline \multirow{5}{*}{$\mathrm{G}_{1}^{\mathrm{S}}$} & absent & $70.7(14.1)$ & 26.8 & 2.4 & $110.0(3.5)$ & 12.4 & 0.6 \\
\hline & $\mathrm{G}_{2}^{\mathrm{B}}$ & $64.6(2.5)$ & 22.5 & 1.5 & $112.9(0.9)$ & 11.8 & 0.4 \\
\hline & $\mathrm{G}_{2}^{S}$ & $72.1(3.0)$ & 20.6 & 0.8 & $80.9(3.9)$ & 12.2 & 0.7 \\
\hline & $\mathrm{G}_{3}^{\mathrm{B}}$ & $69.2(7.7)$ & 22.8 & 1.8 & $106.2(3.0)$ & 12.3 & 0.7 \\
\hline & $\mathrm{G}_{3}^{\mathrm{S}}$ & $62.6(2.7)$ & 17.1 & 1.5 & $111.8(4.2)$ & 12.6 & 0.7 \\
\hline \multirow{5}{*}{$\mathrm{G}_{2}^{S}$} & absent & $77.4(9.6)$ & 26.2 & 2.1 & $111.1(4.9)$ & 15.8 & 1.1 \\
\hline & $\mathrm{G}_{1}^{\mathrm{B}}$ & $89.9(2.8)$ & 21.9 & 0.7 & $110.1(2.1)$ & 13.4 & 0.4 \\
\hline & $\mathrm{G}_{1}^{\mathrm{S}}$ & $94.8(1.0)$ & 17.1 & 1.1 & $95.6(0.5)$ & 10.9 & 0.9 \\
\hline & $\mathrm{G}_{3}^{B}$ & $61.2(14.8)$ & 23.5 & 2.1 & $102.3(1.0)$ & 11.7 & 0.6 \\
\hline & $\mathrm{G}_{3}^{S}$ & $61.8(5.0)$ & 20.4 & 1.8 & $99.6(2.0)$ & 9.4 & 0.3 \\
\hline \multirow{5}{*}{$\mathrm{G}_{3}^{S}$} & absent & $67.6(8.2)$ & 24.2 & 2.6 & 106.1 (1.6) & 11.2 & 0.7 \\
\hline & $\mathrm{G}_{1}^{\mathrm{B}}$ & $59.7(6.5)$ & 19.6 & 1.6 & $110.1(1.6)$ & 10.1 & 0.4 \\
\hline & $\mathrm{G}_{1}^{\mathrm{S}}$ & $57.7(4.9)$ & 18.9 & 1.8 & $111.8(1.5)$ & 13.2 & 0.9 \\
\hline & $\mathrm{G}_{2}^{\mathrm{B}}$ & $68.1(17.4)$ & 26.8 & 2.7 & $108.7(2.5)$ & 11.7 & 0.9 \\
\hline & $\mathrm{G}_{2}^{\mathrm{S}}$ & $93.6(26.7)$ & 30.2 & 3.8 & $103.6(5.0)$ & 13.5 & 0.8 \\
\hline
\end{tabular}

${ }^{\mathrm{a}} \mathrm{Absent}$ indicates monoadducted DNA. ${ }^{\mathrm{b}}$ Average SASA over the three replicates, with the standard deviation (parentheses) calculated between the replicates. 'Standard deviation calculated over the 1.5 $\mu$ s dataset from the three replicas. ${ }^{\mathrm{d}}$ Block standard error (BSE) extrapolated from all frames of the three replicas fitted to a monomolecular growth model. 
Table S21: Occupancies (\%) for the non-Watson-Crick hydrogen bonds at the diadducted DNA lesion sites in the B conformation. ${ }^{\mathrm{a}, \mathrm{b}}$

\begin{tabular}{|c|c|c|c|c|c|c|c|c|c|}
\hline \multirow{2}{*}{ Adduct } & \multirow{2}{*}{$\begin{array}{c}\text { Primary } \\
\text { Lesion }\end{array}$} & \multirow{2}{*}{ Hydrogen Bond ${ }^{c}$} & \multicolumn{7}{|c|}{ Secondary Lesion } \\
\hline & & & None & $\mathrm{G}_{1}-\mathrm{B}$ & $\mathrm{G}_{1}-\mathrm{S}$ & $\mathrm{G}_{2}-\mathrm{B}$ & $\mathrm{G}_{2}-\mathrm{S}$ & $\mathrm{G}_{3}-\mathrm{B}$ & $\mathrm{G}_{3}-\mathrm{S}$ \\
\hline \multirow{5}{*}{${ }^{A N} \mathrm{dG}$} & $\mathrm{G}_{1}^{\mathrm{B}}$ & $(\mathrm{X} 6) \mathrm{N} 10-\mathrm{H} \bullet \bullet \mathrm{O}^{\prime}(\mathrm{C} 5)$ & 29.6 & $\mathrm{~N} / \mathrm{A}$ & $\mathrm{N} / \mathrm{A}$ & 23.1 & 0.0 & 35.5 & 23.1 \\
\hline & $\mathrm{G}_{1}^{B}$ & $(\mathrm{X} 6) \mathrm{N} 10-\mathrm{H} \bullet \bullet \bullet \mathrm{O}^{\prime}(\mathrm{X} 6)$ & 50.3 & $\mathrm{~N} / \mathrm{A}$ & $\mathrm{N} / \mathrm{A}$ & 50.0 & 80.7 & 46.8 & 56.5 \\
\hline & $\mathrm{G}_{2}^{\mathrm{B}}$ & $(\mathrm{X} 7) \mathrm{N} 10-\mathrm{H} \bullet \bullet \bullet 4^{\prime}(\mathrm{X} 7)$ & 79.9 & 79.1 & 69.3 & $\mathrm{~N} / \mathrm{A}$ & $\mathrm{N} / \mathrm{A}$ & 77.1 & 82.5 \\
\hline & $\mathrm{G}_{3}^{B}$ & $(\mathrm{X9}) \mathrm{N} 10-\mathrm{H} \bullet \bullet \mathrm{O}^{\prime}(\mathrm{C} 8)$ & 0.0 & 0.0 & 0.0 & 0.0 & 42.3 & $\mathrm{~N} / \mathrm{A}$ & N/A \\
\hline & $\mathrm{G}_{3}^{B}$ & $(\mathrm{X9}) \mathrm{N} 10-\mathrm{H} \bullet \bullet \bullet 4^{\prime}(\mathrm{X9})$ & 75.0 & 75.1 & 74.0 & 73.6 & 57.2 & $\mathrm{~N} / \mathrm{A}$ & N/A \\
\hline \multirow{3}{*}{${ }^{A P} d G$} & $\mathrm{G}_{1}^{\mathrm{B}}$ & $(\mathrm{X} 6) \mathrm{N} 10-\mathrm{H} \bullet \bullet \mathrm{O}^{\prime}(\mathrm{X} 6)$ & 52.4 & $\mathrm{~N} / \mathrm{A}$ & $\mathrm{N} / \mathrm{A}$ & 81.2 & 65.8 & 53.4 & 50.4 \\
\hline & $\mathrm{G}_{2}^{\mathrm{B}}$ & $(\mathrm{X} 7) \mathrm{N} 10-\mathrm{H} \bullet \bullet \bullet 4^{\prime}(\mathrm{X} 7)$ & 47.9 & 69.1 & 74.9 & $\mathrm{~N} / \mathrm{A}$ & $\mathrm{N} / \mathrm{A}$ & 58.9 & 51.5 \\
\hline & $\mathrm{G}_{3}^{B}$ & (X9)N10-H•••O4'(X9) & 60.4 & 57.3 & 59.5 & 69.3 & 56.7 & N/A & $\mathrm{N} / \mathrm{A}$ \\
\hline
\end{tabular}

${ }^{a}$ Nucleotide numbering is illustrated in Figure $\mathrm{S} 8 .{ }^{\mathrm{b}}$ Occupancies determined as the percent of the simulation that a hydrogen bonds exist according to a heavy atom distance $<3.2 \AA$ and a heavy atomhydrogen-heavy atom angle $>120^{\circ}$. 'X represents the damaged nucleotide. 
Table S22: Average (standard deviation in parentheses) pseudostep parameters ${ }^{\mathrm{a}}$ with in undamaged and diadducted DNA.

\begin{tabular}{|c|c|c|c|c|c|c|c|c|}
\hline Adduct & Sites & Rise (Å) & Shift $(\AA ̊)$ & Slide (Å) & Roll (deg.) & Tilt (deg.) & Twist (deg.) & $\begin{array}{l}\text { Axial Bend } \\
\text { (deg.) }\end{array}$ \\
\hline absent $^{b}$ & N/A & $22.7(0.0)$ & $0.5(0.1)$ & $3.0(0.1)$ & $-9.7(0.5)$ & $0.2(0.7)$ & $-117.0(0.6)$ & $39.4(0.4)$ \\
\hline \multirow{3}{*}{${ }^{\mathrm{AN}} \mathrm{dG}$} & $\mathrm{G}_{1}^{\mathrm{B}} \mathrm{G}_{2}^{\mathrm{B}}$ & $22.3(0.1)$ & $2.7(0.2)$ & $5.1(0.2)$ & $-8.7(0.3)$ & $-9.3(1.2)$ & $-128.0(1.3)$ & $39.4(0.0)$ \\
\hline & $\mathrm{G}_{1}^{\mathrm{B}} \mathrm{G}_{3}^{\mathrm{B}}$ & $22.4(0.0)$ & $1.1(0.1)$ & $4.1(0.1)$ & $-13.5(0.4)$ & $-6.1(0.9)$ & $-122.5(0.3)$ & $38.8(0.2)$ \\
\hline & $\mathrm{G}_{2}^{\mathrm{B}} \mathrm{G}_{3}^{\mathrm{B}}$ & $22.2(0.0)$ & $0.6(0.1)$ & $5.4(0.1)$ & $-18.2(0.4)$ & $-7.3(0.8)$ & $-125.6(0.7)$ & $39.9(0.2)$ \\
\hline \multirow{3}{*}{${ }^{A P} d G$} & $\mathrm{G}_{1}^{\mathrm{B}} \mathrm{G}_{2}^{\mathrm{B}}$ & $22.0(0.1)$ & $3.7(0.1)$ & $6.6(0.1)$ & $-11.2(0.9)$ & $-11.9(0.6)$ & $-135.6(0.6)$ & $40.0(0.0)$ \\
\hline & $\mathrm{G}_{1}^{\mathrm{B}} \mathrm{G}_{3}^{\mathrm{B}}$ & $22.3(0.1)$ & $1.6(0.0)$ & $5.3(0.2)$ & $-10.8(1.3)$ & $-8.8(0.5)$ & $-132.8(0.8)$ & $39.5(0.2)$ \\
\hline & $\mathrm{G}_{2}^{\mathrm{B}} \mathrm{G}_{3}^{\mathrm{B}}$ & $22.2(0.1)$ & $-0.2(0.1)$ & $6.1(0.2)$ & $-19.7(1.9)$ & $-10.2(0.8)$ & $-131.5(0.7)$ & $41.2(0.7)$ \\
\hline \multirow{3}{*}{${ }^{A N} \mathrm{dG}$} & $\mathrm{G}_{1}^{\mathrm{B}} \mathrm{G}_{2}^{\mathrm{S}}$ & $22.2(0.0)$ & $2.0(0.3)$ & $4.3(0.2)$ & $-14.7(3.0)$ & $-9.2(1.8)$ & $-119.7(1.7)$ & $41.0(0.8)$ \\
\hline & $\mathrm{G}_{1}^{\mathrm{B}} \mathrm{G}_{3}^{\mathrm{S}}$ & $21.3(0.5)$ & $-0.8(0.4)$ & $8.3(0.7)$ & $-25.9(1.9)$ & $19.0(0.7)$ & $-132.9(2.0)$ & $50.0(0.7)$ \\
\hline & $\mathrm{G}_{2}^{\mathrm{B}} \mathrm{G}_{3}^{\mathrm{S}}$ & $20.9(0.6)$ & $-1.3(0.8)$ & $8.6(1.6)$ & $-28.0(6.3)$ & $16.8(10.0)$ & $-132.1(2.6)$ & $49.2(4.2)$ \\
\hline \multirow{3}{*}{${ }^{A P} d G$} & $\mathrm{G}_{1}^{\mathrm{B}} \mathrm{G}_{2}^{\mathrm{S}}$ & $24.3(0.1)$ & $0.1(0.1)$ & $-0.8(0.1)$ & $-14.2(1.3)$ & $-9.6(0.8)$ & $-151.2(1.1)$ & $38.5(0.1)$ \\
\hline & $\mathrm{G}_{1}^{\mathrm{B}} \mathrm{G}_{3}^{\mathrm{S}}$ & $20.7(0.3)$ & $-1.3(0.2)$ & $9.7(0.4)$ & $-23.2(1.9)$ & $11.2(1.7)$ & $-140.9(0.9)$ & $44.7(0.9)$ \\
\hline & $\mathrm{G}_{2}^{\mathrm{B}} \mathrm{G}_{3}^{\mathrm{S}}$ & $21.0(0.5)$ & $-2.4(0.2)$ & $8.9(0.9)$ & $-23.8(5.9)$ & $9.0(4.0)$ & $-142.4(6.0)$ & 45.7 (1.3) \\
\hline \multirow{3}{*}{${ }^{A N} \mathrm{dG}$} & $\mathrm{G}_{1}^{\mathrm{S}} \mathrm{G}_{2}^{\mathrm{B}}$ & $20.6(1.1)$ & $3.3(0.9)$ & $8.7(1.0)$ & $-35.9(4.3)$ & $-32.5(1.6)$ & $-127.6(2.2)$ & 54.7 (1.9) \\
\hline & $\mathrm{G}_{1}^{\mathrm{S}} \mathrm{G}_{3}^{\mathrm{B}}$ & $20.1(0.2)$ & $2.8(0.3)$ & $8.9(0.3)$ & $-43.0(2.8)$ & $-28.4(2.7)$ & $-126.9(0.2)$ & $55.0(2.4)$ \\
\hline & $\mathrm{G}_{1}^{\mathrm{S}} \mathrm{G}_{3}^{\mathrm{S}}$ & $21.6(0.8)$ & $0.8(0.6)$ & $6.5(2.0)$ & $-47.3(12.2)$ & $-7.0(1.1)$ & $-125.7(3.6)$ & $55.3(5.2)$ \\
\hline \multirow{3}{*}{${ }^{A P} d G$} & $\mathrm{G}_{1}^{\mathrm{S}} \mathrm{G}_{2}^{\mathrm{B}}$ & $22.0(0.1)$ & $4.7(0.3)$ & $7.0(0.1)$ & $-21.7(1.5)$ & $-10.7(1.0)$ & $-148.4(1.3)$ & $41.8(0.3)$ \\
\hline & $\mathrm{G}_{1}^{\mathrm{S}} \mathrm{G}_{3}^{\mathrm{B}}$ & $21.5(0.2)$ & $2.3(0.1)$ & $6.6(0.4)$ & $-30.5(3.4)$ & $-13.8(0.9)$ & $-139.6(1.7)$ & $47.7(2.5)$ \\
\hline & $\mathrm{G}_{1}^{\mathrm{S}} \mathrm{G}_{3}^{\mathrm{S}}$ & $21.5(0.3)$ & $1.0(0.2)$ & $3.5(6.1)$ & $-27.2(13.4)$ & $-6.8(5.5)$ & $-37.9(179.4)$ & $45.4(10.9)$ \\
\hline \multirow{3}{*}{${ }^{\mathrm{AN}} \mathrm{dG}$} & $\mathrm{G}_{1}^{\mathrm{S}} \mathrm{G}_{2}^{\mathrm{S}}$ & $22.6(0.2)$ & $0.5(1.1)$ & $1.7(1.7)$ & $-17.0(4.5)$ & $-1.3(8.0)$ & $-116.7(0.8)$ & $41.7(0.1)$ \\
\hline & $\mathrm{G}_{1}^{\mathrm{S}} \mathrm{G}_{3}^{\mathrm{S}}$ & $18.3(0.6)$ & $0.8(0.5)$ & $12.6(0.4)$ & $-56.9(1.7)$ & $-1.7(4.2)$ & $-135.8(2.7)$ & $61.5(0.2)$ \\
\hline & $\mathrm{G}_{2}^{\mathrm{S}} \mathrm{G}_{3}^{\mathrm{S}}$ & 22.3 (1.9) & $-2.3(1.3)$ & $10.1(0.3)$ & $-12.8(40.0)$ & $-2.6(14.9)$ & $-47.5(175.1)$ & $46.4(14.2)$ \\
\hline \multirow{3}{*}{${ }^{A P} d G$} & $\mathrm{G}_{1}^{\mathrm{S}} \mathrm{G}_{2}^{\mathrm{S}}$ & $22.9(0.9)$ & $7.5(0.5)$ & $10.6(0.6)$ & $32.3(3.3)$ & $19.4(4.0)$ & $138.2(3.8)$ & $29.5(2.6)$ \\
\hline & $\mathrm{G}_{1}^{\mathrm{S}} \mathrm{G}_{3}^{\mathrm{S}}$ & $20.3(0.0)$ & $-0.1(0.2)$ & $9.3(0.7)$ & $-38.8(3.6)$ & $5.9(0.8)$ & $-144.6(6.2)$ & $50.4(2.1)$ \\
\hline & $\mathrm{G}_{2}^{S} \mathrm{G}_{3}^{S}$ & $22.4(1.1)$ & $-5.2(1.7)$ & $14.5(0.6)$ & $50.3(7.1)$ & $-17.0(5.7)$ & $116.0(8.8)$ & $41.0(4.2)$ \\
\hline
\end{tabular}

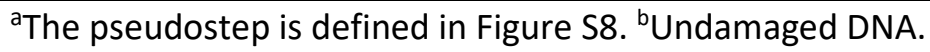


Table S23: Occupancies (\%) for the non-Watson-Crick hydrogen bonds at the diadducted DNA damage sites in the $\mathrm{S}$ conformation. ${ }^{\mathrm{a}, \mathrm{b}}$

\begin{tabular}{|c|c|c|c|c|c|c|c|c|c|}
\hline \multirow{2}{*}{ Adduct } & \multirow{2}{*}{$\begin{array}{l}\text { Primary } \\
\text { Lesion }\end{array}$} & \multirow{2}{*}{ Hydrogen Bond ${ }^{c}$} & \multicolumn{7}{|c|}{ Secondary Lesion } \\
\hline & & & Absent $^{d}$ & $\mathrm{G}_{1}^{B}$ & $\mathrm{G}_{1}^{\mathrm{S}}$ & $\mathrm{G}_{2}^{B}$ & $\mathrm{G}_{2}^{\mathrm{S}}$ & $\mathrm{G}_{3}^{\mathrm{B}}$ & $\mathrm{G}_{3}^{S}$ \\
\hline \multirow{22}{*}{${ }^{A N} \mathrm{dG}$} & \multirow{6}{*}{$\mathrm{G}_{1}^{\mathrm{s}}$} & $(\mathrm{C} 27) \mathrm{N} 4-\mathrm{H} \bullet \bullet \bullet \mathrm{O} 6(\mathrm{X} 6)$ & 21.2 & $\mathrm{~N} / \mathrm{A}$ & $\mathrm{N} / \mathrm{A}$ & 8.0 & 50.3 & 0.0 & 0.0 \\
\hline & & $(\mathrm{X} 6) \mathrm{N} 10-\mathrm{H} \bullet \bullet \cdot \mathrm{N} 1(\mathrm{G} 28)$ & 13.5 & N/A & N/A & 13.7 & 0.0 & 18.1 & 22.9 \\
\hline & & $(\mathrm{X} 6) \mathrm{N} 10-\mathrm{H} \bullet \bullet \cdot \mathrm{N} 2(\mathrm{G} 28)$ & 32.2 & N/A & $\mathrm{N} / \mathrm{A}$ & 49.2 & 0.0 & 47.7 & 48.4 \\
\hline & & $(\mathrm{X} 6) \mathrm{N} 10-\mathrm{H} \bullet \cdots \mathrm{N} 3(\mathrm{G} 28)$ & 0.0 & N/A & $\mathrm{N} / \mathrm{A}$ & 10.8 & 0.0 & 0.0 & 10.3 \\
\hline & & $(\mathrm{X} 6) \mathrm{N} 10-\mathrm{H} \bullet \bullet \bullet 4^{\prime}(\mathrm{X} 7)$ & 17.7 & $\mathrm{~N} / \mathrm{A}$ & N/A & 0.0 & 93.7 & 0.0 & 0.0 \\
\hline & & $(\mathrm{C} 27) \mathrm{N} 4-\mathrm{H} \bullet \bullet \bullet O P 2(\mathrm{C} 26)$ & 7.9 & $\mathrm{~N} / \mathrm{A}$ & $\mathrm{N} / \mathrm{A}$ & 0.0 & 0.0 & 0.0 & 6.3 \\
\hline & \multirow{9}{*}{$\mathrm{G}_{2}^{S}$} & $(\mathrm{C} 26) \mathrm{N} 4-\mathrm{H} \bullet \bullet \bullet \mathrm{OP} 2(\mathrm{G} 25)$ & 0.0 & 6.8 & 0.0 & N/A & $\mathrm{N} / \mathrm{A}$ & 0.0 & 15.8 \\
\hline & & $(\mathrm{C} 26) \mathrm{N} 4-\mathrm{H} \bullet \bullet O 6(X 7)$ & 35.0 & 76.0 & 88.5 & N/A & $\mathrm{N} / \mathrm{A}$ & 9.7 & 15.2 \\
\hline & & $(\mathrm{C} 26) \mathrm{N} 4-\mathrm{H} \bullet \bullet N \mathrm{~N} 7(\mathrm{X} 7)$ & 15.4 & 0.0 & 0.0 & N/A & N/A & 0.0 & 0.0 \\
\hline & & $(\mathrm{X} 6) \mathrm{N} 10-\mathrm{H} \bullet \bullet \mathrm{O}^{\prime}(\mathrm{X} 7)$ & 17.7 & 0.0 & 93.7 & N/A & N/A & 0.0 & 0.0 \\
\hline & & $(\mathrm{X} 7) \mathrm{N} 10-\mathrm{H} \bullet \bullet \mathrm{O}^{\prime}(\mathrm{C} 8)$ & 20.6 & 37.8 & 80.8 & $\mathrm{~N} / \mathrm{A}$ & $\mathrm{N} / \mathrm{A}$ & 10.6 & 0.0 \\
\hline & & $(\mathrm{X} 7) \mathrm{N} 10-\mathrm{H} \bullet \bullet \mathrm{O}^{2}(\mathrm{C} 27)$ & 11.9 & 0.0 & 0.0 & N/A & N/A & 14.2 & 0.0 \\
\hline & & $(\mathrm{X} 7) \mathrm{N} 10-\mathrm{H} \bullet \bullet \bullet \mathrm{N} 2(\mathrm{G} 6)$ & 23.1 & 0.0 & 0.0 & N/A & $\mathrm{N} / \mathrm{A}$ & 25.4 & 36.0 \\
\hline & & $(\mathrm{X} 7) \mathrm{N} 10-\mathrm{H} \bullet \bullet \bullet 4^{\prime}(\mathrm{X} 7)$ & 0.0 & 0.0 & 0.0 & $\mathrm{~N} / \mathrm{A}$ & $\mathrm{N} / \mathrm{A}$ & 0.0 & 17.5 \\
\hline & & $(\mathrm{X} 7) \mathrm{N} 1-\mathrm{H} \bullet \bullet \bullet \mathrm{O}$ (G25) & 0.0 & 8.8 & 12.4 & N/A & N/A & 0.0 & 0.0 \\
\hline & \multirow{7}{*}{$\mathrm{G}_{3}^{\mathrm{S}}$} & $(\mathrm{C} 24) \mathrm{N} 4-\mathrm{H} \bullet \bullet \circ 6(X 9)$ & 12.4 & 9.8 & 9.8 & 16.6 & 53.1 & N/A & N/A \\
\hline & & $(\mathrm{C} 24) \mathrm{N} 4-\mathrm{H} \bullet \bullet N 7(X 9)$ & 0.0 & 0.0 & 0.0 & 0.0 & 21.8 & N/A & N/A \\
\hline & & (X9)N10-H•••O2(C10) & 0.0 & 0.0 & 0.0 & 0.0 & 15.4 & N/A & N/A \\
\hline & & $(\mathrm{X9}) \mathrm{N} 10-\mathrm{H} \bullet \bullet \mathrm{O}^{\prime}(\mathrm{C} 10)$ & 11.3 & 0.0 & 0.0 & 15.2 & 16.8 & $\mathrm{~N} / \mathrm{A}$ & N/A \\
\hline & & $(\mathrm{X} 9) \mathrm{N} 10-\mathrm{H} \bullet \bullet \mathrm{N} 1(\mathrm{G} 25)$ & 0.0 & 9.3 & 9.1 & 0.0 & 0.0 & N/A & N/A \\
\hline & & $(\mathrm{X} 9) \mathrm{N} 10-\mathrm{H} \bullet \bullet \mathrm{N} 2(\mathrm{G} 25)$ & 33.5 & 44.1 & 46.6 & 36.9 & 11.9 & N/A & N/A \\
\hline & & $(\mathrm{X} 9) \mathrm{N} 10-\mathrm{H} \bullet \bullet \cdot \mathrm{N} 3(\mathrm{G} 25)$ & 26.8 & 23.8 & 26.7 & 12.6 & 0.0 & N/A & N/A \\
\hline \multirow{10}{*}{${ }^{A P} \mathrm{dG}$} & \multirow{4}{*}{$\mathrm{G}_{1}^{\mathrm{S}}$} & $(\mathrm{C} 27) \mathrm{N} 4-\mathrm{H} \bullet \bullet O \mathrm{OP} 2(\mathrm{C} 26)$ & 31.3 & $\mathrm{~N} / \mathrm{A}$ & $\mathrm{N} / \mathrm{A}$ & 6.7 & 0.0 & 49.4 & 28.3 \\
\hline & & $(\mathrm{X} 6) \mathrm{N} 10-\mathrm{H} \bullet \bullet \cdot \mathrm{N} 2(\mathrm{G} 28)$ & 14.1 & $\mathrm{~N} / \mathrm{A}$ & N/A & 0.0 & 0.0 & 10.6 & 13.5 \\
\hline & & $(\mathrm{C} 26) \mathrm{N} 4-\mathrm{H} \bullet \bullet O 6(\mathrm{X} 6)$ & 9.9 & $\mathrm{~N} / \mathrm{A}$ & N/A & 0.0 & 0.0 & 0.0 & 0.0 \\
\hline & & $(\mathrm{X} 6) \mathrm{N} 10-\mathrm{H} \bullet \bullet \bullet 4^{\prime}(\mathrm{X} 6)$ & 0.0 & $\mathrm{~N} / \mathrm{A}$ & $\mathrm{N} / \mathrm{A}$ & 0.0 & 90.6 & 0.0 & 0.0 \\
\hline & \multirow{2}{*}{$\mathrm{G}_{2}^{S}$} & $(\mathrm{X} 7) \mathrm{N} 10-\mathrm{H} \bullet \bullet \bullet 4^{\prime}(\mathrm{X} 7)$ & 0.0 & 97.5 & 98.2 & N/A & $\mathrm{N} / \mathrm{A}$ & 11.4 & 95.7 \\
\hline & & $(\mathrm{C} 26) \mathrm{N} 4-\mathrm{H} \bullet \bullet \bullet \mathrm{OP} 2(\mathrm{G} 25)$ & 25.7 & 62.1 & 83.0 & $\mathrm{~N} / \mathrm{A}$ & $\mathrm{N} / \mathrm{A}$ & 40.1 & 0.0 \\
\hline & \multirow{4}{*}{$\mathrm{G}_{3}^{\mathrm{S}}$} & $(\mathrm{C} 24) \mathrm{N} 4-\mathrm{H} \bullet \bullet \bullet O P 2(G 23)$ & 36.7 & 19.4 & 8.7 & 20.0 & 53.5 & $\mathrm{~N} / \mathrm{A}$ & N/A \\
\hline & & $(\mathrm{X} 9) \mathrm{N} 10-\mathrm{H} \bullet \bullet \cdot \mathrm{N} 2(\mathrm{G} 25)$ & 0.0 & 10.4 & 20.2 & 9.6 & 0.0 & N/A & $\mathrm{N} / \mathrm{A}$ \\
\hline & & $(\mathrm{X} 9) \mathrm{N} 10-\mathrm{H} \bullet \bullet \mathrm{N} 3(\mathrm{G} 25)$ & 0.0 & 0.0 & 10.2 & 0.0 & 0.0 & N/A & N/A \\
\hline & & $(\mathrm{X9}) \mathrm{N} 10-\mathrm{H} \bullet \bullet \mathrm{O}^{\prime}(\mathrm{X9})$ & 0.0 & 0.0 & 0.0 & 0.0 & 93.6 & N/A & $\mathrm{N} / \mathrm{A}$ \\
\hline
\end{tabular}

${ }^{a}$ Nucleotide numbering is illustrated in Figure $\mathrm{S} 8 .{ }^{\mathrm{b}}$ Occupancies determined as the percent of the simulation that a hydrogen bonds exist according to a heavy atom distance $<3.2 \AA$ and a heavy atomhydrogen-heavy atom angle $>120^{\circ} .{ }^{c} X$ represents the damaged nucleotide. ${ }^{\mathrm{d}} A$ bsent indicates monoadducted DNA. 
Table S24: Average (standard deviation in parentheses) lesion site interaction energies $(\mathrm{kJ} / \mathrm{mol})$ for monoadducted and diadducted DNA duplexes in the B conformation.

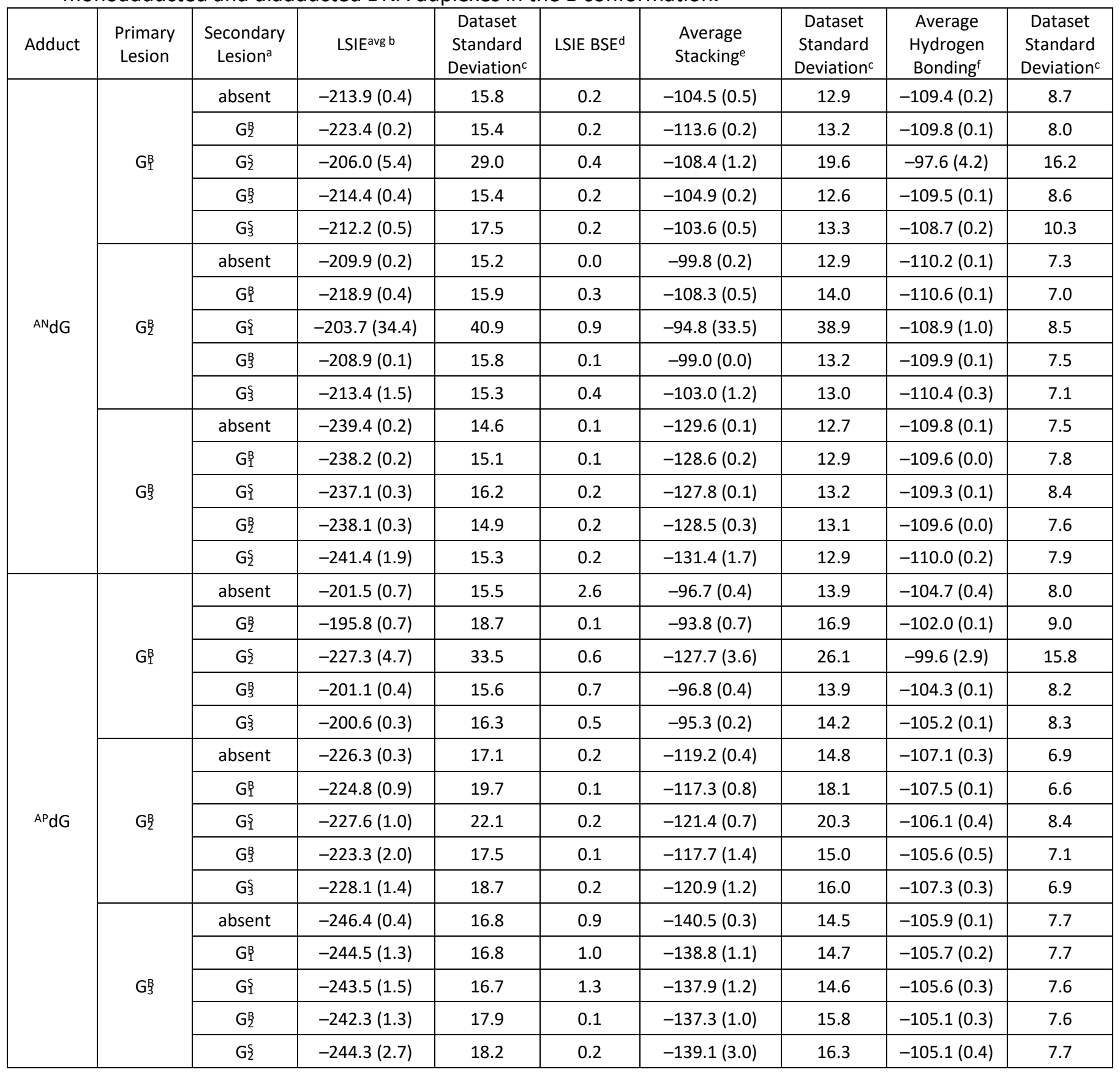

${ }^{\mathrm{a}}$ Absent indicates monoadducted DNA. ${ }^{\mathrm{b}}$ Average of the sum of stacking and hydrogen-bonding interactions for the adducted base, bulky moiety and opposing $\mathrm{C}$, with standard deviation calculated between the replicas. 'Standard deviation calculated over the $1.5 \mu$ s dataset from the three replicas. ${ }^{d}$ Block standard error (BSE) extrapolated from all frames of the three replicas fitted to a monomolecular

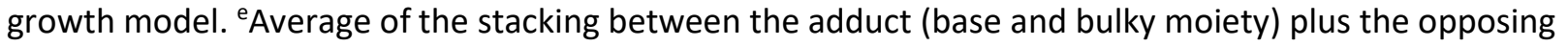
$\mathrm{C}$ and the flanking nucleobases, with standard deviation calculated between the replicas. ${ }^{\mathrm{f}}$ Average of the hydrogen-bonding interaction between the adduct and the pairing $\mathrm{C}$, with standard deviation calculated between the replicas. 
Table S25: Average (standard deviation in parentheses) lesion site interaction energy $(\mathrm{kJ} / \mathrm{mol})$ for monoadducted and diadducted DNA duplexes in the $\mathrm{S}$ conformation.

\begin{tabular}{|c|c|c|c|c|c|c|c|c|c|}
\hline Adduct & $\begin{array}{l}\text { Primary } \\
\text { Lesion }\end{array}$ & $\begin{array}{l}\text { Secondary } \\
\text { Lesion }^{\mathrm{a}}\end{array}$ & LSIEavg, b & $\begin{array}{c}\text { Dataset } \\
\text { Standard } \\
\text { Deviation }{ }^{c}\end{array}$ & LSIE BSE ${ }^{d}$ & Average Stackinge & $\begin{array}{c}\text { Dataset } \\
\text { Standard } \\
\text { Deviation }{ }^{c}\end{array}$ & $\begin{array}{c}\text { Average } \\
\text { Hydrogen } \\
\text { Bonding } \\
\end{array}$ & $\begin{array}{c}\text { Dataset } \\
\text { Standard } \\
\text { Deviation }\end{array}$ \\
\hline \multirow{15}{*}{$\mathrm{AN}_{\mathrm{dG}}$} & \multirow{5}{*}{$\mathrm{G}_{1}^{\mathrm{s}}$} & absent & $-24.1(65.1)$ & 74.9 & 1.7 & $-40.0(35.8)$ & 44.4 & $15.9(29.3)$ & 33.5 \\
\hline & & $\mathrm{G}_{2}^{\mathrm{B}}$ & $-50.9(89.2)$ & 96.0 & 2.4 & $-58.0(48.4)$ & 55.6 & $7.1(40.8)$ & 42.7 \\
\hline & & $\mathrm{G}_{2}^{S}$ & $-20.2(66.9)$ & 74.5 & 1.8 & $-25.9(45.1)$ & 51.4 & $5.7(21.8)$ & 25.5 \\
\hline & & $\mathrm{G}_{3}^{B}$ & $25.5(1.8)$ & 27.4 & 0.5 & $-13.6(1.2)$ & 24.4 & $39.1(0.9)$ & 8.3 \\
\hline & & $\mathrm{G}_{3}^{S}$ & $19.9(4.1)$ & 36.4 & 0.7 & $-17.2(4.0)$ & 29.2 & $37.1(0.4)$ & 11.2 \\
\hline & \multirow{5}{*}{$\mathrm{G}_{2}^{S}$} & absent & $-33.7(25.1)$ & 72.0 & 1.5 & $-48.7(15.0)$ & 46.2 & $15.1(10.1)$ & 30.1 \\
\hline & & $\mathrm{G}_{1}^{\mathrm{B}}$ & $-67.7(1.8)$ & 37.7 & 0.5 & $-78.0(1.5)$ & 28.4 & $10.3(0.2)$ & 17.2 \\
\hline & & $\mathrm{G}_{1}^{\mathrm{S}}$ & $-27.6(38.7)$ & 55.8 & 1.0 & $-39.8(37.3)$ & 46.4 & $12.3(1.5)$ & 18.0 \\
\hline & & $\mathrm{G}_{3}^{B}$ & $2.4(36.2)$ & 57.2 & 1.2 & $-25.2(22.0)$ & 37.7 & $27.6(14.2)$ & 23.1 \\
\hline & & $\mathrm{G}_{3}^{S}$ & $-156.8(18.3)$ & 48.2 & 1.3 & $-117.3(9.1)$ & 29.5 & $-39.5(9.3)$ & 21.8 \\
\hline & \multirow{5}{*}{$\mathrm{G}_{3}^{S}$} & absent & $-39.1(64.2)$ & 91.3 & 2.1 & $-47.9(39.7)$ & 57.5 & $8.9(24.6)$ & 36.5 \\
\hline & & $\mathrm{G}_{1}^{\mathrm{B}}$ & $-18.0(87.0)$ & 92.8 & 2.3 & $-28.2(44.2)$ & 50.4 & $10.3(42.8)$ & 44.8 \\
\hline & & $\mathrm{G}_{1}^{\mathrm{S}}$ & $-23.4(88.8)$ & 95.0 & 2.4 & $-31.9(45.6)$ & 51.5 & $8.5(43.2)$ & 45.8 \\
\hline & & $\mathrm{G}_{2}^{\mathrm{B}}$ & $21.5(36.4)$ & 61.4 & 1.3 & $-10.5(22.9)$ & 40.5 & $32.1(13.5)$ & 24.4 \\
\hline & & $\mathrm{G}_{2}^{S}$ & $-117.3(26.0)$ & 59.8 & 1.3 & $-83.4(11.7)$ & 35.9 & $-33.9(15.5)$ & 28.1 \\
\hline \multirow{15}{*}{$\mathrm{AP}^{\mathrm{d}} \mathrm{G}$} & \multirow{5}{*}{$\mathrm{G}_{1}^{S}$} & absent & $-123.3(36.2)$ & 51.6 & 1.1 & $-103.4(21.6)$ & 33.7 & $-19.9(15.1)$ & 23.8 \\
\hline & & $\mathrm{G}_{2}^{\mathrm{B}}$ & $-85.2(1.6)$ & 25.6 & 0.2 & $-82.4(1.4)$ & 24.2 & $-2.8(0.3)$ & 3.5 \\
\hline & & $\mathrm{G}_{2}^{S}$ & $-93.9(7.0)$ & 32.1 & 0.5 & $-81.8(9.1)$ & 31.2 & $-12.1(2.7)$ & 9.0 \\
\hline & & $\mathrm{G}_{3}^{\mathrm{B}}$ & $-77.6(5.5)$ & 46.0 & 1.0 & $-78.4(3.7)$ & 32.3 & $0.8(1.8)$ & 16.0 \\
\hline & & $\mathrm{G}_{3}^{\mathrm{S}}$ & $-119.8(39.7)$ & 56.9 & 1.2 & $-103.7(25.1)$ & 39.5 & $-16.1(14.6)$ & 22.5 \\
\hline & \multirow{5}{*}{$\mathrm{G}_{2}^{S}$} & absent & $-101.2(7.4)$ & 49.6 & 0.8 & $-106.8(4.4)$ & 37.6 & $5.6(3.6)$ & 16.1 \\
\hline & & $\mathrm{G}_{1}^{\mathrm{B}}$ & $-138.2(2.4)$ & 22.7 & 0.2 & $-135.2(3.1)$ & 23.4 & $-3.0(0.8)$ & 4.7 \\
\hline & & $\mathrm{G}_{1}^{\mathrm{S}}$ & $-113.0(10.6)$ & 40.0 & 1.1 & $-105.8(10.6)$ & 38.6 & $-7.1(0.4)$ & 4.9 \\
\hline & & $\mathrm{G}_{3}^{B}$ & $-108.4(3.8)$ & 41.0 & 0.7 & $-110.3(3.4)$ & 32.9 & $1.9(2.2)$ & 11.6 \\
\hline & & $\mathrm{G}_{3}^{S}$ & $-159.0(17.4)$ & 34.7 & 0.8 & $-144.5(16.5)$ & 33.6 & $-14.6(1.4)$ & 5.5 \\
\hline & \multirow{5}{*}{$\mathrm{G}_{3}^{S}$} & absent & $-81.5(7.1)$ & 49.4 & 1.3 & $-86.7(4.9)$ & 37.8 & $5.2(2.3)$ & 13.9 \\
\hline & & $\mathrm{G}_{1}^{\mathrm{B}}$ & $-56.0(9.7)$ & 49.3 & 0.7 & $-67.9(7.0)$ & 37.3 & $12.0(2.8)$ & 13.9 \\
\hline & & $\mathrm{G}_{1}^{\mathrm{S}}$ & $-83.5(10.4)$ & 69.8 & 1.7 & $-79.6(4.2)$ & 43.7 & $-3.8(6.2)$ & 30.8 \\
\hline & & $\mathrm{G}_{2}^{\mathrm{B}}$ & $-63.6(12.5)$ & 50.3 & 0.9 & $-72.9(9.2)$ & 38.0 & $9.3(3.3)$ & 14.4 \\
\hline & & $\mathrm{G}_{2}^{\mathrm{S}}$ & $-115.3(15.2)$ & 37.4 & 1.1 & $-112.2(15.9)$ & 38.1 & $-3.1(0.8)$ & 6.0 \\
\hline
\end{tabular}

${ }^{\mathrm{a}}$ Absent indicates monoadducted DNA. ${ }^{\mathrm{b}}$ Average of the sum of stacking and hydrogen-bonding interactions for the adducted base, bulky moiety and opposing $\mathrm{C}$, with standard deviation calculated between the replicas. 'Standard deviation calculated over the $1.5 \mu$ s dataset from the three replicas. ${ }^{d}$ Block standard error (BSE) extrapolated from all frames of the three replicas fitted to a monomolecular growth model. eAverage of the stacking between the adduct (base and bulky moiety) plus the opposing $\mathrm{C}$ and the flanking nucleobases, with standard deviation calculated between the replicas. ${ }^{\mathrm{f}}$ Average of the hydrogen-bonding interaction between the adduct and the pairing $\mathrm{C}$, with standard deviation calculated between the replicas. 
Table S26: Average $\Delta\left(\right.$ SASA $\left.^{\text {avg }}\right)$ and $\Delta\left(\right.$ LSIE $\left.^{\text {avg }}\right)$ upon the addition of a second lesion.

\begin{tabular}{|c|c|c|c|c|c|}
\hline \multirow[b]{2}{*}{$\begin{array}{l}\text { Primary } \\
\text { Lesion }\end{array}$} & \multirow{2}{*}{$\begin{array}{l}\text { Secondary } \\
\text { Lesion }^{\mathrm{a}}\end{array}$} & \multicolumn{2}{|c|}{${ }^{\mathrm{AN}} \mathrm{dG}$} & \multicolumn{2}{|c|}{${ }^{A P} \mathrm{dG}$} \\
\hline & & $\begin{array}{c}\Delta\left(\mathrm{SASA}^{\text {avg }}\right)^{\mathrm{b}} \\
\left(\AA^{2}\right)\end{array}$ & $\begin{array}{c}\Delta\left(\text { LSIE }^{\text {avg }}\right)^{c} \\
(\mathrm{~kJ} / \mathrm{mol})\end{array}$ & $\begin{array}{c}\Delta\left(\text { SASA }^{\text {avg }}\right)^{b} \\
\left(\AA^{2}\right)\end{array}$ & $\begin{array}{c}\Delta\left(\text { LSIE }^{\text {avg }}\right)^{\mathrm{c}} \\
(\mathrm{kJ} / \mathrm{mol})\end{array}$ \\
\hline \multirow{5}{*}{$\mathrm{G}_{1}^{\mathrm{B}}$} & absent & 0.0 & 0.0 & 0.0 & 0.0 \\
\hline & $\mathrm{G}_{2}^{\mathrm{B}}$ & -15.1 & -9.5 & -22.8 & 5.7 \\
\hline & $\mathrm{G}_{2}^{S}$ & -2.8 & 7.8 & -42.3 & -25.8 \\
\hline & $\mathrm{G}_{3}^{\mathrm{B}}$ & -0.1 & -0.5 & 0.6 & 0.4 \\
\hline & $\mathrm{G}_{3}^{\mathrm{S}}$ & 0.2 & 1.7 & -2.7 & 0.9 \\
\hline \multirow{5}{*}{$\mathrm{G}_{2}^{\mathrm{B}}$} & absent & 0.0 & 0.0 & 0.0 & 0.0 \\
\hline & $\mathrm{G}_{1}^{\mathrm{B}}$ & -22.1 & -8.9 & -31.1 & 1.6 \\
\hline & $\mathrm{G}_{1}^{\mathrm{S}}$ & -12.2 & 6.2 & -18.3 & -1.2 \\
\hline & $\mathrm{G}_{3}^{\mathrm{B}}$ & 1.5 & 1.0 & -7.7 & 3.1 \\
\hline & $\mathrm{G}_{3}^{\mathrm{S}}$ & -1.5 & -3.5 & -0.6 & -1.8 \\
\hline \multirow{5}{*}{$\mathrm{G}_{3}^{B}$} & absent & 0.0 & 0.0 & 0.0 & 0.0 \\
\hline & $\mathrm{G}_{1}^{\mathrm{B}}$ & -0.1 & 1.2 & 1.2 & 1.9 \\
\hline & $\mathrm{G}_{1}^{\mathrm{S}}$ & -0.7 & 2.3 & 1.5 & 2.9 \\
\hline & $\mathrm{G}_{2}^{\mathrm{B}}$ & -0.2 & 1.3 & -12.5 & 4.1 \\
\hline & $\mathrm{G}_{2}^{\mathrm{S}}$ & 0.4 & -2.0 & -4.5 & 2.1 \\
\hline \multirow{5}{*}{$\mathrm{G}_{1}^{\mathrm{s}}$} & absent & 0.0 & 0.0 & 0.0 & 0.0 \\
\hline & $\mathrm{G}_{2}^{\mathrm{B}}$ & -6.2 & -26.8 & 2.9 & 38.1 \\
\hline & $\mathrm{G}_{2}^{\mathrm{S}}$ & 1.4 & 4.0 & -29.1 & 29.4 \\
\hline & $\mathrm{G}_{3}^{\mathrm{B}}$ & -1.6 & 49.6 & -3.9 & 45.7 \\
\hline & $\mathrm{G}_{3}^{\mathrm{s}}$ & -8.1 & 44.0 & 1.8 & 3.5 \\
\hline \multirow{5}{*}{$\mathrm{G}_{2}^{S}$} & absent & 0.0 & 0.0 & 0.0 & 0.0 \\
\hline & $\mathrm{G}_{1}^{\mathrm{B}}$ & 12.6 & -34.0 & -1.0 & -37.1 \\
\hline & $\mathrm{G}_{1}^{\mathrm{S}}$ & 17.5 & 6.1 & -15.5 & -11.8 \\
\hline & $\mathrm{G}_{3}^{\mathrm{B}}$ & -16.1 & 36.1 & -8.7 & -7.2 \\
\hline & $\mathrm{G}_{3}^{S}$ & -15.6 & -123.1 & -11.5 & -57.8 \\
\hline \multirow{5}{*}{$\mathrm{G}_{3}^{S}$} & absent & 0.0 & 0.0 & 0.0 & 0.0 \\
\hline & $\mathrm{G}_{1}^{\mathrm{B}}$ & -7.9 & 21.1 & 4.0 & 25.5 \\
\hline & $\mathrm{G}_{1}^{\mathrm{S}}$ & -9.9 & 15.7 & 5.7 & -1.9 \\
\hline & $\mathrm{G}_{2}^{\mathrm{B}}$ & 0.5 & 60.6 & 2.6 & 17.9 \\
\hline & $\mathrm{G}_{2}^{S}$ & 26.0 & -78.2 & -2.5 & -33.7 \\
\hline
\end{tabular}

${ }^{a}$ Absent indicates monoadducted DNA. ${ }^{b} \Delta\left(S A S A^{\text {avg }}\right)$ denotes the $S A S A^{\text {avg }}$ of a given adduct site in the presence of a second lesion (diadducted DNA) minus the SASA ${ }^{\text {avg }}$ of the same adduct site in the absence of a second lesion (monoadducted DNA). ${ }^{c}\left(\operatorname{LSIE}^{\text {avg }}\right.$ ) denotes the $\mathrm{LSIE}^{\text {avg }}$ of a given adduct site in the presence of a second lesion (diadducted DNA) minus the LSIE ${ }^{\text {avg }}$ of the same adduct site in the absence of a second lesion (monoadducted DNA). 
Table S27: MOL2 file containing partial charges and atom types of the ${ }^{A N}$ dG adduct used for MD simulations (atom numbers are provided in the associated figure).

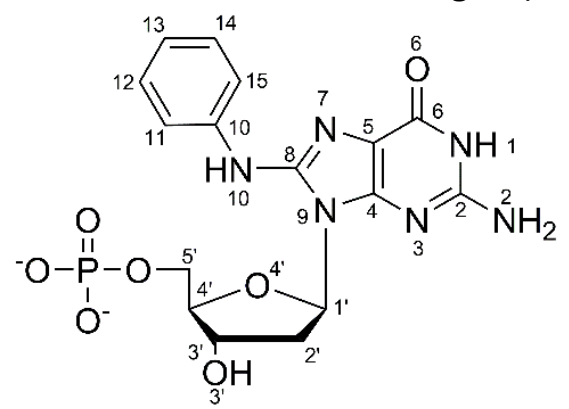

$\begin{array}{lllllllll}1 & \mathrm{P} & 4.7050 & 0.0520 & -1.1680 & \mathrm{P} & 1 & \text { LIG } & 1.2090 \\ 2 & \mathrm{O} 5^{\prime} & 4.1730 & 1.0760 & 0.0000 & \mathrm{OS} & 1 & \text { LIG } & -0.4900 \\ 3 & \mathrm{OP} 1 & 4.6090 & 0.7320 & -2.4680 & \mathrm{O} 2 & 1 & \text { LIG } & -0.7864 \\ 4 & \mathrm{OP2} & 5.9220 & -0.5810 & -0.6380 & \mathrm{O} 2 & 1 & \text { LIG } & -0.7953 \\ 5 & \mathrm{O} 3 & 0.0000 & 0.0000 & 0.0000 & \mathrm{OS} & 1 & \text { LIG } & -0.5474 \\ 6 & \mathrm{C} 5^{\prime} & 3.1010 & 1.9490 & -0.3030 & \mathrm{CI} & 1 & \text { LIG } & -0.0102 \\ 7 & \mathrm{H} 5^{\prime} & 2.8330 & 2.4680 & 0.6250 & \mathrm{H} 1 & 1 & \text { LIG } & 0.0752 \\ 8 & \mathrm{H} 5^{\prime \prime} & 3.3910 & 2.7140 & -1.0420 & \mathrm{H} 1 & 1 & \text { LIG } & 0.0752 \\ 9 & \mathrm{C} 4^{\prime} & 1.8690 & 1.2180 & -0.8380 & \mathrm{CT} & 1 & \text { LIG } & 0.1830 \\ 10 & \mathrm{H} 4^{\prime} & 1.0270 & 1.9170 & -0.9140 & \mathrm{H} 1 & 1 & \text { LIG } & 0.0978 \\ 11 & \mathrm{O} 4^{\prime} & 2.1700 & 0.7140 & -2.1590 & \mathrm{OS} & 1 & \text { LIG } & -0.4082 \\ 12 & \mathrm{C} 1^{\prime} & 1.9220 & -0.6850 & -2.2280 & \mathrm{CT} & 1 & \text { LIG } & 0.1596 \\ 13 & \mathrm{H} 1^{\prime} & 0.9300 & -0.8780 & -2.6450 & \mathrm{H} 2 & 1 & \text { LIG } & 0.0902 \\ 14 & \mathrm{C} 3^{\prime} & 1.4280 & 0.0000 & 0.0000 & \mathrm{CT} & 1 & \text { LIG } & 0.1484 \\ 15 & \mathrm{H} 3^{\prime} & 1.8240 & 0.0530 & 1.0220 & \mathrm{H} 1 & 1 & \text { LIG } & 0.0720 \\ 16 & \mathrm{C} 2^{\prime} & 2.0070 & -1.1870 & -0.7800 & \mathrm{CT} & 1 & \text { LIG } & -0.0617 \\ 17 & \mathrm{H} 2^{\prime} & 3.0480 & -1.3520 & -0.4920 & \mathrm{HC} & 1 & \text { LIG } & 0.0466 \\ 18 & \mathrm{H} 2^{\prime \prime} & 1.4440 & -2.1140 & -0.6290 & \mathrm{HC} & 1 & \text { LIG } & 0.0466 \\ 19 & \mathrm{~N} 9 & 2.8790 & -1.2770 & -3.1530 & \mathrm{~N} * & 1 & \text { LIG } & -0.0563 \\ 20 & \mathrm{C} 8 & 2.6100 & -2.0090 & -4.3140 & \mathrm{CR} & 1 & \text { LIG } & 0.4678 \\ 21 & \mathrm{C} 4 & 4.2540 & -1.2720 & -3.0400 & \mathrm{CB} & 1 & \text { LIG } & 0.1484 \\ 22 & \mathrm{~N} 7 & 3.6880 & -2.4790 & -4.8900 & \mathrm{NB} & 1 & \text { LIG } & -0.5254 \\ 23 & \mathrm{C} 5 & 4.7300 & -2.0310 & -4.1010 & \mathrm{CB} & 1 & \text { LIG } & 0.0762 \\ 24 & \mathrm{~N} 4 & 4.9730 & -0.6260 & -2.0850 & \mathrm{NC} & 1 & \text { LIG } & -0.5421 \\ 25 & \mathrm{C6} & 6.1500 & -2.2180 & -4.2500 & \mathrm{C} & 1 & \text { LIG } & 0.5106 \\ 26 & \mathrm{C} 2 & 6.2750 & -0.7780 & -2.1980 & \mathrm{CA} & 1 & \text { LIG } & 0.6729 \\ 27 & \mathrm{O} 6 & 6.7970 & -2.8430 & -5.0700 & \mathrm{O} & 1 & \text { LIG } & -0.5407 \\ 28 & \mathrm{~N} 1 & 6.8450 & -1.5130 & -3.1980 & \mathrm{NA} & 1 & \text { LIG } & -0.4810 \\ 29 & \mathrm{H} 1 & 7.8500 & -1.6540 & -3.2180 & \mathrm{H} & 1 & \text { LIG } & 0.3501 \\ 30 & \mathrm{~N} 2 & 7.0960 & -0.2220 & -1.2480 & \mathrm{~N} 2 & 1 & \text { LIG } & -0.8752 \\ 31 & \mathrm{H} 21 & 8.0220 & 0.0670 & -1.5350 & \mathrm{H} & 1 & \text { LIG } & 0.3884 \\ 32 & \mathrm{H} 22 & 6.6240 & 0.4340 & -0.6340 & \mathrm{H} & 1 & \text { LIG } & 0.3884\end{array}$




$\begin{array}{lllllllll}33 & \mathrm{~N} 10 & 1.3100 & -2.3020 & -4.7270 & \mathrm{~N} 2 & 1 & \text { LIG } & -0.5894 \\ 34 & \mathrm{H} 10 & 1.3080 & -3.1770 & -5.2390 & \mathrm{H} & 1 & \text { LIG } & 0.3511 \\ 35 & \mathrm{C} 10 & 0.4170 & -1.3450 & -5.2830 & \mathrm{CA} & 1 & \text { LIG } & 0.2156 \\ 36 & \mathrm{C} 11 & 0.6440 & 0.0360 & -5.2250 & \mathrm{CA} & 1 & \text { LIG } & -0.0547 \\ 37 & \mathrm{H} 11 & 1.5430 & 0.4310 & -4.7640 & \mathrm{HA} & 1 & \text { LIG } & 0.1212 \\ 38 & \mathrm{C} 15 & -0.7540 & -1.8230 & -5.8900 & \mathrm{CA} & 1 & \text { LIG } & -0.2053 \\ 39 & \mathrm{H} 15 & -0.9360 & -2.8950 & -5.9310 & \mathrm{HA} & 1 & \text { LIG } & 0.1458 \\ 40 & \mathrm{C} 12 & -0.2970 & 0.9160 & -5.7630 & \mathrm{CA} & 1 & \text { LIG } & -0.2343 \\ 41 & \mathrm{H} 12 & -0.1060 & 1.9850 & -5.7080 & \text { HA } & 1 & \text { LIG } & 0.1637 \\ 42 & \mathrm{C} 14 & -1.6780 & -0.9360 & -6.4340 & \mathrm{CA} & 1 & \text { LIG } & -0.1747 \\ 43 & \mathrm{H} 14 & -2.5760 & -1.3250 & -6.9060 & \mathrm{HA} & 1 & \text { LIG } & 0.1548 \\ 44 & \mathrm{C} 13 & -1.4580 & 0.4430 & -6.3720 & \mathrm{CA} & 1 & \text { LIG } & -0.1124 \\ 45 & \mathrm{H} 13 & -2.1820 & 1.1350 & -6.7910 & \text { HA } & 1 & \text { LIG } & 0.1321\end{array}$

Table S28: MOL2 file containing partial charges and atom types of the AP dG adduct used for MD simulations (atom numbers are provided in the associated figure).

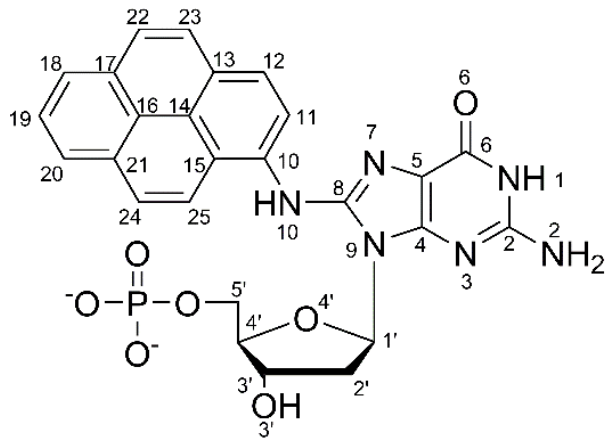

\begin{tabular}{|c|c|c|c|c|c|c|c|c|}
\hline 1 & $P$ & 4.9760 & 0.3860 & -1.3260 & $P$ & 1 & LIG & 1.1662 \\
\hline 2 & O5' & 4.1390 & 0.9990 & 0.0000 & OS & 1 & LIG & -0.4956 \\
\hline 3 & OP1 & 5.1120 & 1.4640 & -2.3580 & $\mathrm{O} 2$ & 1 & LIG & -0.7681 \\
\hline 4 & OP2 & 6.1010 & -0.4220 & -0.7550 & $\mathrm{O} 2$ & 1 & LIG & -0.7681 \\
\hline 5 & $\mathrm{O} 3$ & 0.0000 & 0.0000 & 0.0000 & OS & 1 & LIG & -0.5424 \\
\hline 6 & C5' & 3.1040 & 1.8950 & -0.2820 & $\mathrm{Cl}$ & 1 & LIG & -0.0196 \\
\hline 7 & H5' & 3.4230 & 2.6620 & -0.9830 & $\mathrm{H} 1$ & 1 & LIG & 0.0821 \\
\hline 8 & H5" & 2.8550 & 2.3890 & 0.6500 & $\mathrm{H} 1$ & 1 & LIG & 0.0821 \\
\hline 9 & C4' & 1.8570 & 1.2140 & -0.8200 & CT & 1 & LIG & 0.1633 \\
\hline 10 & H4' & 1.0480 & 1.9310 & -0.8780 & $\mathrm{H} 1$ & 1 & LIG & 0.1054 \\
\hline 11 & O4' & 2.1080 & 0.7240 & -2.1370 & OS & 1 & LIG & -0.4032 \\
\hline 12 & $C 1^{\prime}$ & 1.8650 & -0.6500 & -2.2260 & CT & 1 & LIG & 0.1513 \\
\hline 13 & $\mathrm{H} 1^{\prime}$ & 0.8780 & -0.8250 & -2.6240 & $\mathrm{H} 2$ & 1 & LIG & 0.0873 \\
\hline 14 & C3' & 1.4070 & 0.0000 & 0.0000 & CT & 1 & LIG & 0.1347 \\
\hline 15 & H3' & 1.7910 & 0.0400 & 1.0120 & $\mathrm{H} 1$ & 1 & LIG & 0.0784 \\
\hline 16 & $C 2^{\prime}$ & 1.9750 & -1.1690 & -0.7950 & CT & 1 & LIG & -0.0572 \\
\hline 17 & $\mathrm{H} 2^{\prime}$ & 3.0130 & -1.3300 & -0.5360 & $\mathrm{HC}$ & 1 & LIG & 0.0443 \\
\hline 18 & H2" & 1.4270 & -2.0920 & -0.6530 & $\mathrm{HC}$ & 1 & LIG & 0.0443 \\
\hline
\end{tabular}




\begin{tabular}{|c|c|c|c|c|c|c|c|c|}
\hline 19 & N9 & 2.7970 & -1.2560 & -3.1630 & $\mathrm{~N}^{*}$ & 1 & LIG & -0.0519 \\
\hline 20 & C8 & 4.0990 & -0.9300 & -3.5110 & CR & 1 & LIG & 0.3777 \\
\hline 21 & $\mathrm{C} 4$ & 2.5500 & -2.4460 & -3.7900 & $\mathrm{CB}$ & 1 & LIG & 0.1445 \\
\hline 22 & N7 & 4.6490 & -1.7930 & -4.2750 & NB & 1 & LIG & -0.4566 \\
\hline 23 & $\mathrm{C} 5$ & 3.6890 & -2.7690 & -4.4570 & $\mathrm{CB}$ & 1 & LIG & 0.0570 \\
\hline 24 & N3 & 1.3850 & -3.1370 & -3.7080 & NC & 1 & LIG & -0.4990 \\
\hline 25 & C6 & 3.7200 & -3.9790 & -5.2240 & $C$ & 1 & LIG & 0.4977 \\
\hline 26 & $\mathrm{C} 2$ & 1.3980 & -4.2210 & -4.4050 & $\mathrm{CA}$ & 1 & LIG & 0.5921 \\
\hline 27 & 06 & 4.5820 & -4.4690 & -5.8910 & 0 & 1 & LIG & -0.5456 \\
\hline 28 & N1 & 2.4720 & -4.6420 & -5.1190 & NA & 1 & LIG & -0.4138 \\
\hline 29 & $\mathrm{H} 1$ & 2.3970 & -5.4580 & -5.6890 & $\mathrm{H}$ & 1 & LIG & 0.3351 \\
\hline 30 & N2 & 0.2780 & -4.9950 & -4.4810 & $\mathrm{~N} 2$ & 1 & LIG & -0.8656 \\
\hline 31 & $\mathrm{H} 21$ & 0.3970 & -5.9770 & -4.5990 & $\mathrm{H}$ & 1 & LIG & 0.3895 \\
\hline 32 & $\mathrm{H} 22$ & -0.4320 & -4.7410 & -3.8300 & $\mathrm{H}$ & 1 & LIG & 0.3895 \\
\hline 33 & N10 & 4.6780 & 0.2390 & -3.0360 & N2 & 1 & LIG & -0.3628 \\
\hline 34 & $\mathrm{H} 10$ & 4.0050 & 0.9680 & -2.9720 & $\mathrm{H}$ & 1 & LIG & 0.2783 \\
\hline 35 & C10 & 5.9550 & 0.6490 & -3.5100 & $C A$ & 1 & LIG & 0.0814 \\
\hline 36 & C15 & 6.1060 & 1.8360 & -4.2310 & CA & 1 & LIG & 0.0633 \\
\hline 37 & C11 & 7.0640 & -0.1160 & -3.1790 & $C A$ & 1 & LIG & -0.2616 \\
\hline 38 & $\mathrm{H} 11$ & 6.9210 & -1.0300 & -2.6350 & $\mathrm{HA}$ & 1 & LIG & 0.1751 \\
\hline 39 & C14 & 7.4130 & 2.2570 & -4.5810 & $C A$ & 1 & LIG & 0.0181 \\
\hline 40 & $\mathrm{C} 23$ & 4.9910 & 2.6640 & -4.6450 & $C A$ & 1 & LIG & -0.1706 \\
\hline 41 & $\mathrm{H} 23$ & 3.9870 & 2.3450 & -4.4390 & $\mathrm{HA}$ & 1 & LIG & 0.1296 \\
\hline 42 & $\mathrm{C} 12$ & 8.3300 & 0.2810 & -3.5480 & $C A$ & 1 & LIG & -0.1240 \\
\hline 43 & $\mathrm{H} 12$ & 9.1750 & -0.3300 & -3.2860 & $\mathrm{HA}$ & 1 & LIG & 0.1527 \\
\hline 44 & C16 & 7.6070 & 3.4880 & -5.2930 & $C A$ & 1 & LIG & 0.0859 \\
\hline 45 & C13 & 8.5310 & 1.4720 & -4.2390 & $C A$ & 1 & LIG & 0.0232 \\
\hline 46 & $\mathrm{C} 22$ & 5.1770 & 3.8110 & -5.3090 & $C A$ & 1 & LIG & -0.2254 \\
\hline 47 & $\mathrm{H} 22^{\prime}$ & 4.3310 & 4.4020 & -5.6120 & $\mathrm{HA}$ & 1 & LIG & 0.1573 \\
\hline 48 & $\mathrm{C} 21$ & 6.4980 & 4.2810 & -5.6550 & $C A$ & 1 & LIG & 0.0462 \\
\hline 49 & $\mathrm{C} 17$ & 8.9100 & 3.9110 & -5.6360 & $C A$ & 1 & LIG & 0.0488 \\
\hline 50 & $\mathrm{C} 24$ & 9.8500 & 1.9270 & -4.6100 & $C A$ & 1 & LIG & -0.1744 \\
\hline 51 & $\mathrm{H} 24$ & 10.6920 & 1.3130 & -4.3430 & $\mathrm{HA}$ & 1 & LIG & 0.1419 \\
\hline 52 & $\mathrm{C} 20$ & 6.7030 & 5.4740 & -6.3400 & $\mathrm{CA}$ & 1 & LIG & -0.1411 \\
\hline 53 & $\mathrm{H} 20$ & 5.8560 & 6.0770 & -6.6170 & HA & 1 & LIG & 0.1383 \\
\hline 54 & C18 & 9.0760 & 5.1110 & -6.3220 & $C A$ & 1 & LIG & -0.1473 \\
\hline 55 & H18 & 10.0680 & 5.4350 & -6.5840 & $\mathrm{HA}$ & 1 & LIG & 0.1409 \\
\hline 56 & $\mathrm{C} 25$ & 10.0320 & 3.0790 & -5.2670 & $\mathrm{CA}$ & 1 & LIG & -0.1962 \\
\hline 57 & $\mathrm{H} 25$ & 11.0200 & 3.4070 & -5.5350 & $\mathrm{HA}$ & 1 & LIG & 0.1453 \\
\hline 58 & C19 & 7.9830 & 5.8840 & -6.6680 & $C A$ & 1 & LIG & -0.2217 \\
\hline 59 & H19 & 8.1290 & 6.8080 & -7.1990 & $\mathrm{HA}$ & 1 & LIG & 0.1629 \\
\hline
\end{tabular}

